\title{
Oxidative Allylic Esterification of Alkenes by Cooperative Selenium-Catalysis using Air as the Sole Oxidant
}

\author{
Stefan Ortgies, Christian Depken and Alexander Breder* \\ Institut für Organische und Biomolekulare Chemie, Georg-August-Universität \\ Göttingen, Tammannstr. 2, D-37077 Göttingen, Germany.
}

\section{Supporting Information}

Table of Contents

General Remarks

Optimization of the reaction conditions

Synthesis and analytical data of alkene substrates 1 and photocatalyst 4

Synthesis and analytical data of acyloxylation products 3

Selenofunctionalization and elimination experiments

Interrupted irradiation experiment

Fluorescence quenching experiments

${ }^{1} \mathrm{H}-/{ }^{13} \mathrm{C}-/ / \mathrm{R}-\mathrm{spectra}$

S28 


\section{General Remarks}

Catalytic reactions were carried out under an air or oxygen atmosphere, except stated otherwise. Chemicals were obtained from commercial sources and were used without further purification. Yields correspond to isolated compounds unless indicated otherwise. Purity is estimated to be $\geq 95 \%$ based on ${ }^{1} \mathrm{H}-\mathrm{NMR}$ spectroscopic analysis. Irradiation experiments were performed at $\lambda=465 \mathrm{~nm}$ using commercially available blue LED strips (2835 super bright SMD-LEDs, 100 diodes/m, 400 Im/m, 24 V, 12 W; see experimental setup picture below). The light intensity applied was in the range of 3500-4500 Ix (approximate distance of the flask from the LED strips was $1-3 \mathrm{~cm}$ ). Reaction vessels used were made of DURAN glass. TLC: MACHEREY-NAGEL, TLC plates Alugram ${ }^{\circledR}$ Sil G/UV254. Visualization of the developed chromatogram was performed by fluorescence quenching at $254 \mathrm{~nm}$ and staining with potassium permanganate. Chromatography: Separations were carried out on Merck Silica 60 (0.063-0.200 mm, 70-230 mesh ASTM) using forced flow. IR: Bruker FT-IR Alphaspectrometer with ATR sampling module. High resolution mass spectrometry (HRMS): APEX IV 7T FTICR, BRUKER Daltonic. NMR $\left({ }^{1} \mathrm{H},{ }^{13} \mathrm{C}\right)$ spectra were recorded at $300,400,500 \mathrm{MHz}\left({ }^{1} \mathrm{H}\right)$ and $101,126 \mathrm{MHz}\left({ }^{13} \mathrm{C}\right.$, APT (Attached Proton Test)), respectively, on VARIAN Unity-300, AMX 300 and Inova-500 instruments in $\mathrm{CDCl}_{3}$ solutions, if not otherwise specified. Chemical shifts $(\delta)$ are given in ppm. Multiplicity ( $s$ = singlet,$\quad d$ =doublet,$\quad t=$ triplet,$\quad q=$ quartet,$\quad$ quint $=$ quintet,$\quad$ sex = sextet , sept $=$ septet,$\quad m=$ multiplet. Fluorescence measurements were performed on a JASCO FP-6500 spectrofluorometer at $20^{\circ} \mathrm{C}$. The excitation wavelength was $\lambda=465 \mathrm{~nm}$. The emission was recorded at $\lambda=539 \mathrm{~nm}$. 


\section{Optimization of the reaction conditions}

To a solution of benzyl (E)-hex-3-enoate (102 mg, $500 \mu \mathrm{mol}, 1.00$ equiv.) in an appropriate solvent and acetic acid (0.1 M) under an air atmosphere are added $(\mathrm{PhSe})_{2}$ and 2,4,6-tris(4-methoxyphenyl)pyrylium tetrafluoroborate ((p-anisyl) $\left.)_{3} \mathrm{Pyr}, 4\right)$. The resulting mixture is vigorously stirred at ambient temperature under irradiation at $465 \mathrm{~nm}$ for $16 \mathrm{~h}$. The solvent is removed under reduced pressure and the yield is determined by ${ }^{1} \mathrm{H}$-NMR-spectroscopy using $1,3,5$-trimethoxybenzene as the internal standard.

Table S1: Solvent optimization

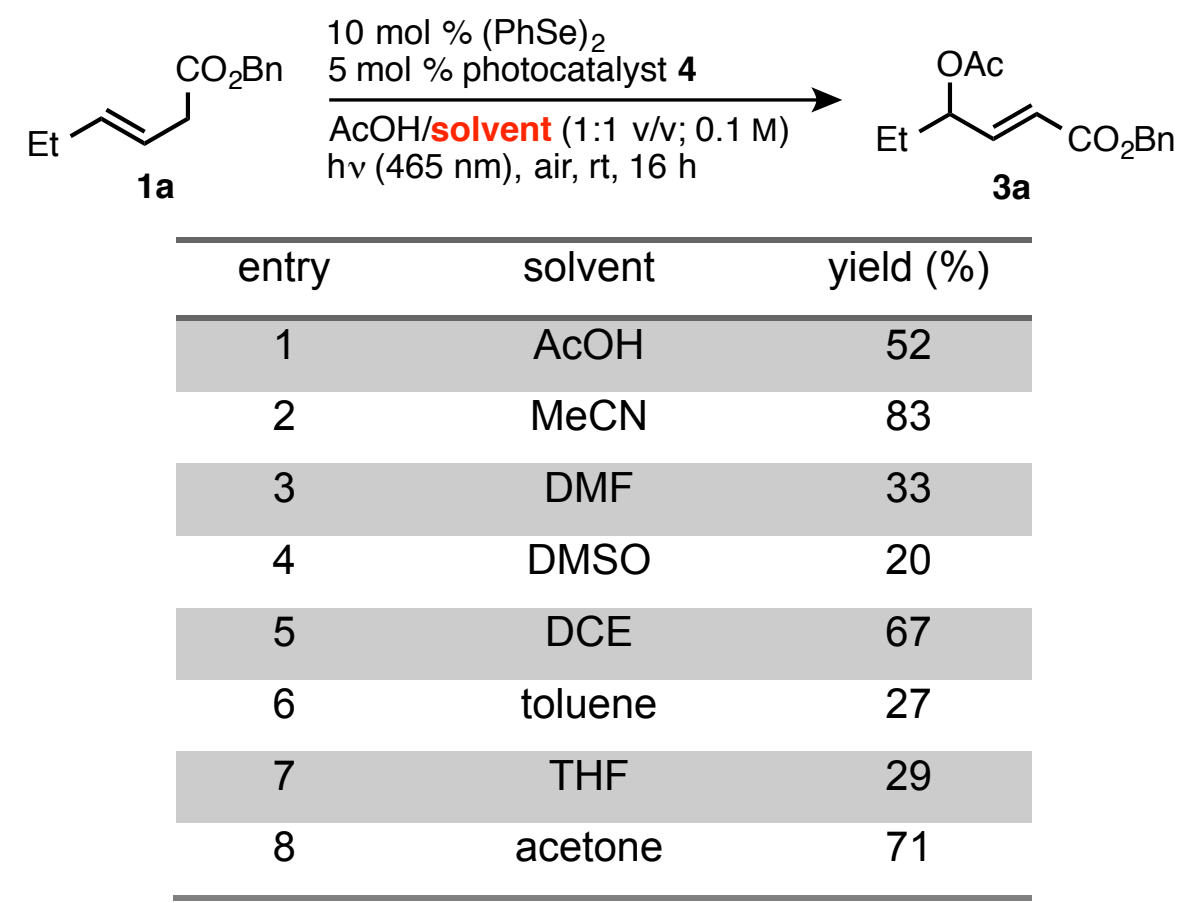


Table S2: Solvent/acid ratio and concentration optimization

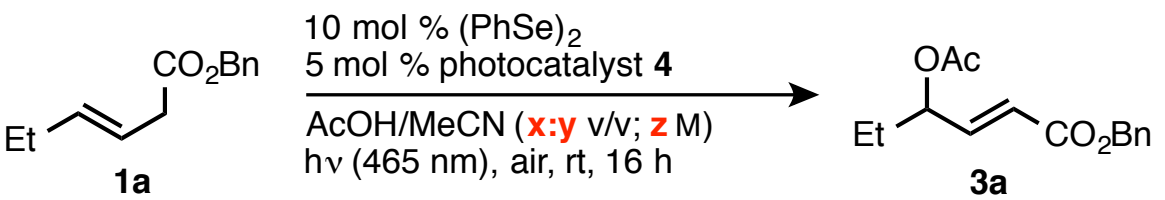

\begin{tabular}{cccc}
\hline entry & concentration $(\mathrm{M})$ & $\mathbf{x} / \mathbf{y}$ volume ratio & yield $(\%)$ \\
\hline 1 & 0.1 & $1: 1$ & 83 \\
2 & 0.1 & $2: 3$ & $>95$ \\
3 & 0.1 & $3: 7$ & 72 \\
4 & 0.1 & $1: 4$ & 61 \\
5 & 0.1 & $1: 9$ & 50 \\
6 & 0.1 & $1: 19$ & 26 \\
7 & 0.2 & $2: 3$ & $>95$ \\
8 & 0.5 & $2: 3$ & 89 \\
\hline
\end{tabular}

Table S3: Catalyst loading optimization and control experiments

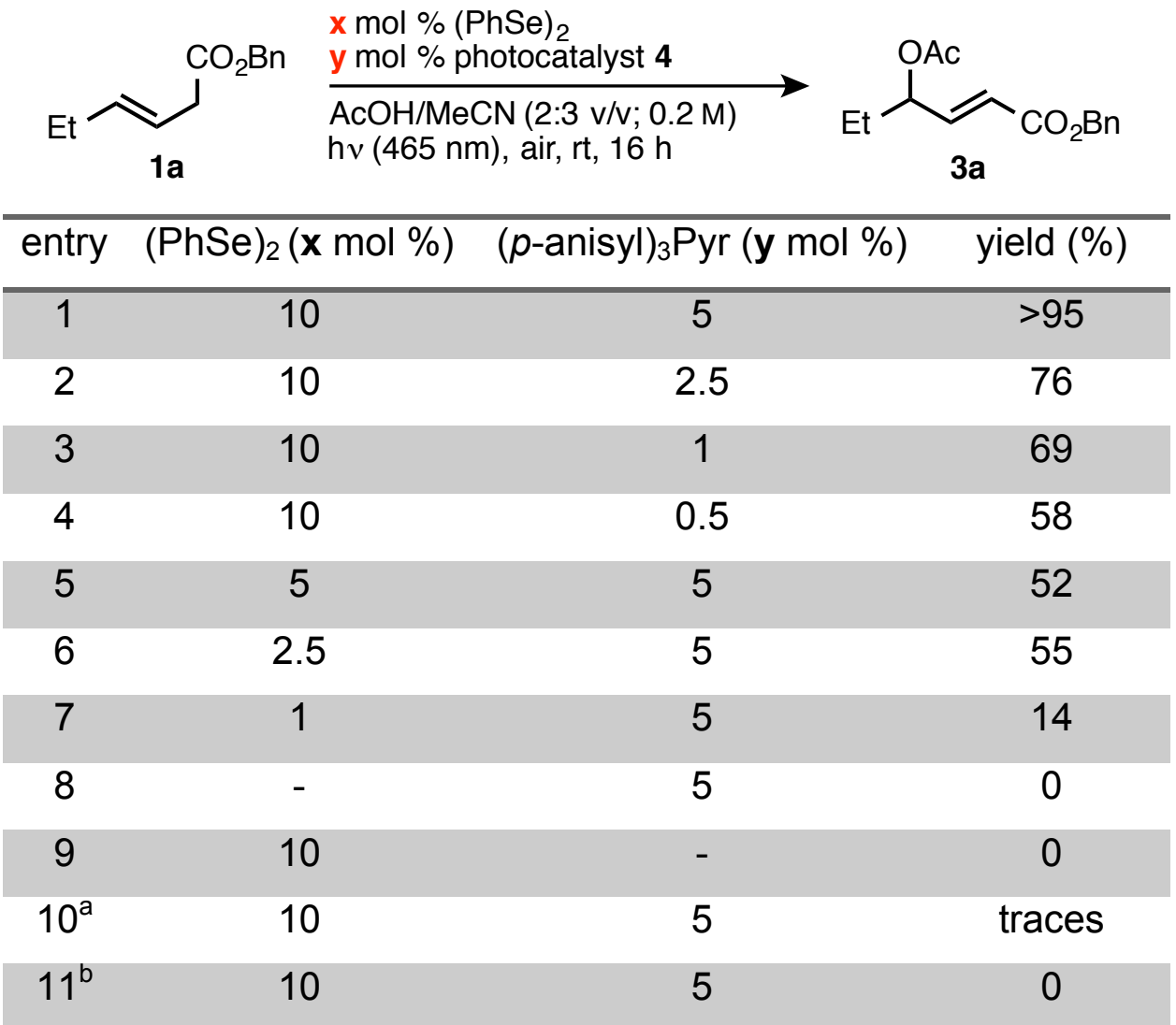

[a] Reaction was conducted under an argon atmosphere using degassed solvents; [b] reaction was conducted in the dark. 


\section{Synthesis and analytical data of alkene substrates and photocatalyst}

\section{2,4,6-Tris(4-methoxyphenyl)pyrylium tetrafluoroborate (4)}

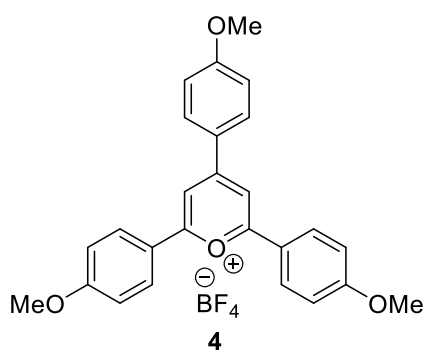

To a solution of 4'-methoxyacetophenone (15.0 g, $100 \mathrm{mmol}, 2.0$ equiv.) and freshly distilled 4-methoxybenzaldehyde ( $6.08 \mathrm{~mL}, 6.81 \mathrm{~g}, 50.0 \mathrm{mmol}, 1.0$ equiv.) in dry toluene $(5 \mathrm{~mL})$ under an argon atmosphere is slowly adde $\mathrm{BF}_{3} \cdot \mathrm{Et}_{2} \mathrm{O}(14.8 \mathrm{~mL}, 17.0 \mathrm{~g}, 120 \mathrm{mmol}, 2.4$ equiv.) and the resulting mixture is stirred at $100{ }^{\circ} \mathrm{C}$ for $2 \mathrm{~h}$. The formed $\mathrm{Et}_{2} \mathrm{O}$ is removed under reduced pressure and the residue is dissolved in acetone. $\mathrm{Et}_{2} \mathrm{O}$ is added and the formed precipitate is filtered off and recrystallized from acetone.

IR (neat): $v=2941,2841,1585,1482,1457,1434,1258,1235,1174,1016,829$, 562, $518 \mathrm{~cm}^{-1}$; ${ }^{1} \mathrm{H}-\mathrm{NMR}\left(300 \mathrm{MHz}, \mathrm{DMSO}-\mathrm{D}_{6}\right): \delta(\mathrm{ppm})=3.91(\mathrm{~s}, 6 \mathrm{H}), 3.94(\mathrm{~s}, 3 \mathrm{H})$, 7.04-7.21 (m, $6 \mathrm{H}), 8.29$ (d, J = 9.0 Hz, $4 \mathrm{H}$ ), $8.43(\mathrm{~d}, J=9.1 \mathrm{~Hz}, 2 \mathrm{H}), 8.54(\mathrm{~s}, 2 \mathrm{H})$; ${ }^{13} \mathrm{C}$-NMR $\left.(126 \mathrm{MHz} \text {, DMSO-D })_{6}\right):(\mathrm{ppm})=55.8,55.9,110.3,115.1,115.2,121.0$, 124.2, 130.4, 132.2, 161.5, 164.4, 165.2, 167.4; HR-MS (ESI): $\left[\mathrm{C}_{26} \mathrm{H}_{23} \mathrm{O}_{4}\right]^{+}([\mathrm{M}-$ $\left.\mathrm{BF}_{4}^{-}\right]^{+}$): obs.: 399.1587 ; calcd.: 399.1591 .

\section{Benzyl (E)-hex-3-enoate (1a)}

$\mathrm{C}_{1 \mathrm{a}}^{\mathrm{OBn}}$ To a solution of $(E)$-hex-3-enoic acid $(3.12 \mathrm{~mL}, 3.00 \mathrm{~g}, 26.3 \mathrm{mmol}$, benzyl alcohol (5.47 mL, $5.68 \mathrm{~g}, 52.6 \mathrm{mmol}, 2.0$ equiv.) and $p-\mathrm{TsOH} \cdot \mathrm{H}_{2} \mathrm{O}(250 \mathrm{mg}$, $1.31 \mathrm{mmol}, 0.05$ equiv.). The resulting mixture is stirred at $150{ }^{\circ} \mathrm{C}$ for $16 \mathrm{~h}$. The solution is allowed to cool to ambient temperature, diluted with EtOAc $(50 \mathrm{~mL})$ and washed with sat. aqueous $\mathrm{NaHCO}_{3}$-solution $(3 \times 50 \mathrm{~mL})$ and brine $(50 \mathrm{~mL})$. The aqueous layer is back extracted with EtOAc $(50 \mathrm{~mL})$. The combined organic layers are dried over $\mathrm{Na}_{2} \mathrm{SO}_{4}$, the solid is filtered off and the solvents are removed under reduced pressure. The residue is purified by column chromatography on silica gel using $n$-pentane/EtOAc (10:1) as the eluent. The desired compound is obtained as a colourless liquid (5.35 g, $26.2 \mathrm{mmol}$, >99 \%). 
DC: $R_{f}=0.54$ ( $n$-pentane/EtOAc, 10:1); IR (neat): $v=3034,2963,1734,1455,1233$, 1151, 966, 734, $695 \mathrm{~cm}^{-1} ;{ }^{1} \mathrm{H}-\mathrm{NMR}\left(300 \mathrm{MHz}, \mathrm{CDCl}_{3}\right): \delta(\mathrm{ppm})=0.99$ (t, $J=7.4 \mathrm{~Hz}$, $3 \mathrm{H}), 1.94-2.15(\mathrm{~m}, 2 \mathrm{H}), 2.96-3.21(\mathrm{~m}, 2 \mathrm{H}), 5.12(\mathrm{~s}, 2 \mathrm{H}), 5.45-5.72(\mathrm{~m}, 2 \mathrm{H}), 7.24-$ $7.45(\mathrm{~m}, 5 \mathrm{H}) ;{ }^{13} \mathrm{C}-\mathrm{NMR}\left(75 \mathrm{MHz}, \mathrm{CDCl}_{3}\right): \delta(\mathrm{ppm})=13.4,25.5,38.0,66.2,120.4$, 128.1, 128.1, 128.5, 135.9, 136.4, 172.0; HR-MS (ESI): $\left[\mathrm{C}_{13} \mathrm{H}_{16} \mathrm{NaO}_{2}\right]^{+}\left([\mathrm{M}+\mathrm{Na}]^{+}\right)$: obs.: 227.1045 ; calcd.: 227.1043 .

\section{Phenyl (E)-pent-3-enoate (1b)}

Me OPh To a solution of $(E)$-pent-3-enoic acid $(2.03 \mathrm{~mL}, 2.00 \mathrm{~g}, 20.0 \mathrm{mmol}$, 1b 1.0 equiv.) in toluene $(20 \mathrm{~mL})$ in a DEAN-STARK apparatus are added phenol (3.76 g, $40.0 \mathrm{mmol}, 2.0$ equiv.) and $p-\mathrm{TsOH} \cdot \mathrm{H}_{2} \mathrm{O}(190 \mathrm{mg}, 1.00 \mathrm{mmol}$, 0.05 equiv.). The resulting mixture is stirred at $150{ }^{\circ} \mathrm{C}$ for $48 \mathrm{~h}$. The solution is allowed to cool to ambient temperature, diluted with EtOAc $(50 \mathrm{~mL})$ and washed with sat. aqueous $\mathrm{NaHCO}_{3}$-solution $(3 \times 50 \mathrm{~mL})$ and brine $(50 \mathrm{~mL})$. The aqueous layer is back extracted with EtOAc $(50 \mathrm{~mL})$. The combined organic layers are dried over $\mathrm{Na}_{2} \mathrm{SO}_{4}$, the solid is filtered off and the solvents are removed under reduced pressure. The residue is purified by column chromatography on silica gel using $n$ pentane/EtOAc $(10: 1)$ as the eluent. The desired compound is obtained as a colourless liquid (2.54 g, $14.4 \mathrm{mmol}, 72 \%)$.

DC: $R_{f}=0.46$ (n-pentane/EtOAc, 10:1); IR (neat): $v=2919,1753,1592,1492,1192$, $1161,1127,964,928,892,814,746,688,500 \mathrm{~cm}^{-1} ;{ }^{1} \mathrm{H}-\mathrm{NMR}\left(300 \mathrm{MHz}, \mathrm{CDCl}_{3}\right): \delta$ $(\mathrm{ppm})=1.51-1.88(\mathrm{~m}, 3 \mathrm{H}), 3.26(\mathrm{dd}, J=5.4,1.3 \mathrm{~Hz}, 2 \mathrm{H}), 5.22-5.80(\mathrm{~m}, 2 \mathrm{H}), 7.03-$ $7.12(\mathrm{~m}, 2 \mathrm{H}), 7.21(\mathrm{~m}, 1 \mathrm{H}), 7.31-7.41(\mathrm{~m}, 2 \mathrm{H}) ;{ }^{13} \mathrm{C}-\mathrm{NMR}\left(126 \mathrm{MHz}, \mathrm{CDCl}_{3}\right): \delta$ $(\mathrm{ppm})=17.9,38.1,121.5,122.1,125.7,129.3,130.1,150.7,170.5$; HR-MS (ESI): $\left[\mathrm{C}_{11} \mathrm{H}_{13} \mathrm{O}_{2}\right]^{+}\left([\mathrm{M}+\mathrm{H}]^{+}\right)$: obs.: 177.0905; calcd.: 177.0910 .

\section{Hexyl (E)-hex-3-enoate (1c)}

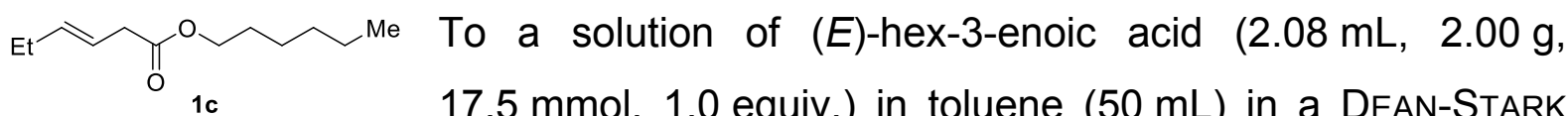
apparatus are added 1-hexanol $(4.37 \mathrm{~mL}, 3.58 \mathrm{~g}, 35.0 \mathrm{mmol}, 2.0$ equiv.) and $p-\mathrm{TsOH} \cdot \mathrm{H}_{2} \mathrm{O}$ (167 mg, $876 \mu \mathrm{mol}, 0.05$ equiv.). The resulting mixture is stirred at $150{ }^{\circ} \mathrm{C}$ for $16 \mathrm{~h}$. The solution is allowed to cool to ambient temperature, diluted with EtOAc $(50 \mathrm{~mL})$ and washed with sat. aqueous $\mathrm{NaHCO}_{3}$-solution $(3 \times 50 \mathrm{~mL})$ and brine $(50 \mathrm{~mL})$. The aqueous layer is back extracted with EtOAc $(50 \mathrm{~mL})$. The 
combined organic layers are dried over $\mathrm{Na}_{2} \mathrm{SO}_{4}$, the solid is filtered off and the solvents are removed under reduced pressure. The residue is purified by column chromatography on silica gel using $n$-pentane/EtOAc $(10: 1)$ as the eluent. The desired compound is obtained as a colourless liquid $(2.38 \mathrm{~g}, 12.0 \mathrm{mmol}, 69 \%)$.

DC: $R_{f}=0.52$ ( $n$-pentane/EtOAc, 10:1); IR (neat): $v=2958,2930,2858,1736,1460$, 1241, 1160, $966 \mathrm{~cm}^{-1}$; ${ }^{1} \mathrm{H}-\mathrm{NMR}\left(300 \mathrm{MHz}, \mathrm{CDCl}_{3}\right): \delta(\mathrm{ppm})=0.80-0.91(\mathrm{~m}, 3 \mathrm{H}), 0.97$ $(\mathrm{t}, J=7.5 \mathrm{~Hz}, 3 \mathrm{H}), 1.20-1.40(\mathrm{~m}, 6 \mathrm{H}), 1.52-1.67(\mathrm{~m}, 2 \mathrm{H}), 1.96-2.10(\mathrm{~m}, 2 \mathrm{H}), 2.94-$ $3.05(\mathrm{~m}, 2 \mathrm{H}), 4.05(\mathrm{t}, J=6.7 \mathrm{~Hz}, 2 \mathrm{H}), 5.39-5.67(\mathrm{~m}, 2 \mathrm{H}) ;{ }^{13} \mathrm{C}-\mathrm{NMR}(126 \mathrm{MHz}$, $\left.\mathrm{CDCl}_{3}\right): \delta(\mathrm{ppm})=13.5,14.0,22.6,25.6,25.6,28.6,31.5,38.2,64.7,120.6,136.1$, 172.2; HR-MS (ESI): [ $\left.\mathrm{C}_{12} \mathrm{H}_{22} \mathrm{NaO}_{2}\right]^{+}\left([\mathrm{M}+\mathrm{Na}]^{+}\right)$: obs.: 221.1518; calcd.: 221.1512.

\section{Benzyl (E)-4-phenylbut-3-enoate (1d)}

OBn To a solution of $(E)$-4-phenylbut-3-enoic acid $(500 \mathrm{mg}, 3.08 \mathrm{mmol}$, ${ }_{1 d}{ }^{\circ} 1.0$ equiv.) in toluene $(25 \mathrm{~mL})$ in a DEAN-STARK apparatus are added benzyl alcohol $\left(480 \mu \mathrm{L}, 500 \mathrm{mg}, 4.62 \mathrm{mmol}, 1.5\right.$ equiv.) and $p-\mathrm{TsOH} \cdot \mathrm{H}_{2} \mathrm{O}(29.3 \mathrm{mg}$, $154 \mu \mathrm{mol}, 0.05$ equiv.). The resulting mixture is stirred at $150{ }^{\circ} \mathrm{C}$ for $16 \mathrm{~h}$. The solution is allowed to cool to ambient temperature, diluted with EtOAc $(50 \mathrm{~mL})$ and washed with sat. aqueous $\mathrm{NaHCO}_{3}$-solution $(3 \times 50 \mathrm{~mL})$ and brine $(50 \mathrm{~mL})$. The aqueous layer is back extracted with EtOAc $(50 \mathrm{~mL})$. The combined organic layers are dried over $\mathrm{Na}_{2} \mathrm{SO}_{4}$, the solid is filtered off and the solvents are removed under reduced pressure. The residue is purified by column chromatography on silica gel using $n$-pentane/ $\mathrm{Et}_{2} \mathrm{O}(20: 1)$ as the eluent. The desired compound is obtained as a white solid (621 mg, $2.46 \mathrm{mmol}, 80 \%$ ).

DC: $R_{f}=0.15$ (n-pentane/ $\mathrm{Et}_{2} \mathrm{O}, 20: 1$ ); m.p.: $35-36{ }^{\circ} \mathrm{C}$; IR (neat): $v=3029,1731$, 1496, 1450, 1236, 1145, 963, 734, 692, $494 \mathrm{~cm}^{-1} ;{ }^{1} \mathrm{H}-\mathrm{NMR}\left(400 \mathrm{MHz}, \mathrm{CDCl}_{3}\right): \delta$ $(\mathrm{ppm})=3.29(\mathrm{dd}, J=7.0,1.4 \mathrm{~Hz}, 2 \mathrm{H}), 5.15(\mathrm{~s}, 2 \mathrm{H}), 6.31(\mathrm{dt}, J=15.9,7.0 \mathrm{~Hz}, 1 \mathrm{H})$, $6.49(\mathrm{dt}, J=15.9,1.4 \mathrm{~Hz}, 1 \mathrm{H}), 7.19-7.40(\mathrm{~m}, 10 \mathrm{H}) ;{ }^{13} \mathrm{C}-\mathrm{NMR}\left(101 \mathrm{MHz}, \mathrm{CDCl}_{3}\right): \delta$ $(\mathrm{ppm})=38.4$, 66.6, 121.5, 126.3, 127.5, 128.2, 128.3, 128.5, 128.6, 133.6, 135.8, 136.8, 171.4; HR-MS (ESI): $\left[\mathrm{C}_{17} \mathrm{H}_{16} \mathrm{NaO}_{2}\right]^{+}\left([\mathrm{M}+\mathrm{Na}]^{+}\right)$: obs.: 275.1044 ; calcd.: 275.1043. 


\section{(E)-Hept-3-enenitrile (1e)}

Me $\Upsilon_{1 \mathrm{e}} \widehat{C N}_{\mathrm{CN}}$ To a mixture of $\mathrm{Nal}(1.80 \mathrm{~g}, 12.0 \mathrm{mmol}, 1.2$ equiv.) in MeCN (15 mL) is added TMSCl $(1.53 \mathrm{~mL}, 1.31 \mathrm{~g}, 12.0 \mathrm{mmol}, 1.2$ equiv.) dropwise at ambient temperature. (E)-Hex-2-ene-1-ol (1.18 mL, $1.00 \mathrm{~g}, 10.0 \mathrm{mmol}, 1.0$ equiv.) is added and the resulting mixture is stirred for $30 \mathrm{~min}$ at ambient temperature. KCN ( $1.95 \mathrm{~g}, 30.0 \mathrm{mmol}, 3.0$ equiv.) is added and stirring is continued for $16 \mathrm{~h} . \mathrm{H}_{2} \mathrm{O}$ $(10 \mathrm{~mL})$ is added and the mixture is extracted with $\mathrm{Et}_{2} \mathrm{O}(3 \times 15 \mathrm{~mL})$. The combined organic layers are washed with $10 \%$ aqueous $\mathrm{Na}_{2} \mathrm{~S}_{2} \mathrm{O}_{3}$-solution $(15 \mathrm{~mL})$ and dried over $\mathrm{Na}_{2} \mathrm{SO}_{4}$. The solid is filtered off and the solvents are removed under reduced pressure. The residue is purified by column chromatography on silica gel using $n$ pentane/EtOAc $(10: 1)$ as the eluent. The desired compound is obtained as a colourless liquid (675 mg, $6.18 \mathrm{mmol}, 62 \%$ ).

DC: $R_{f}=0.56$ ( $n$-pentane/EtOAc, 10:1); IR (neat): $v=2961,2931,2874,2251,1459$, 1418, 1380, $968 \mathrm{~cm}^{-1} ;{ }^{1} \mathrm{H}-\mathrm{NMR}\left(300 \mathrm{MHz}, \mathrm{CDCl}_{3}\right): \delta(\mathrm{ppm})=0.89(\mathrm{t}, J=7.3 \mathrm{~Hz}, 3 \mathrm{H})$, 1.40 (sext, $J=7.3 \mathrm{~Hz}, 2 \mathrm{H}$ ), $2.03(\mathrm{dt}, J=7.3,7.2 \mathrm{~Hz}, 2 \mathrm{H}$ ), 3.05 (dd, $J=7.3,0.6 \mathrm{~Hz}$, $2 \mathrm{H}), 5.32(\mathrm{~m}, 1 \mathrm{H}), 5.81(\mathrm{~m}, 1 \mathrm{H}) ;{ }^{13} \mathrm{C}$-NMR $\left(126 \mathrm{MHz}, \mathrm{CDCl}_{3}\right): \delta(\mathrm{ppm})=13.5,20.4$, 21.9, 34.1, 117.1, 117.8, 136.1; HR-MS $(E \mathrm{El}):\left[\mathrm{C}_{7} \mathrm{H}_{10} \mathrm{~N}\right]^{+}\left([\mathrm{M}-\mathrm{H}]^{++}\right)$: obs.: 108.0814; calcd.: 108.0813.

\section{Diethyl (E)-but-2-en-1-ylphosphonate (1f)}

Me $ح_{\mathrm{PO}(\mathrm{OEt})_{2}}$ To a solution of diethyl phosphonate $(1.05 \mathrm{~mL}, 1.13 \mathrm{~g}, 8.15 \mathrm{mmol}$, 1.1 equiv.) in dry THF ( $25 \mathrm{~mL})$ under an argon atmosphere is added $n$-butyl lithium ( $2.5 \mathrm{M}, 3.26 \mathrm{~mL}, 522 \mathrm{mg}, 8.15 \mathrm{mmol}, 1.1$ equiv.) dropwise at $-10{ }^{\circ} \mathrm{C}$ and the mixture is stirred for $10 \mathrm{~min}$. A solution of crotyl bromide ( $85 \%$ trans, $762 \mu \mathrm{L}$, $1.00 \mathrm{~g}, 7.41 \mathrm{mmol}, 1.0$ equiv.) in dry THF $(2 \mathrm{~mL})$ is added dropwise and the reaction mixture is stirred at $-10^{\circ} \mathrm{C}$ for 30 min. Sat. aqueous $\mathrm{NH}_{4} \mathrm{Cl}$-solution $(30 \mathrm{~mL})$ is added and the mixture is extracted with DCM $(3 \times 50 \mathrm{~mL})$. The combined organic layers are washed with brine $(50 \mathrm{~mL})$ and dried over $\mathrm{Na}_{2} \mathrm{SO}_{4}$. The solid is filtered off and the solvents are removed under reduced pressure. The residue is purified by column chromatography on silica gel using $n$-pentane/ $\mathrm{Et}_{2} \mathrm{O}(2: 1)$ as the eluent. The desired compound is obtained as a colourless liquid $(1.04 \mathrm{~g}, 5.39 \mathrm{mmol}, 73 \%, 85: 15 \mathrm{E} / \mathrm{Z})$.

DC: $R_{f}=0.14$ ( $n$-pentane/EtOAc, 2:1); IR (neat): $v=3471,2981,1392,1248,1021$, 956, 777, $519 \mathrm{~cm}^{-1} ;{ }^{1} \mathrm{H}-\mathrm{NMR}\left(500 \mathrm{MHz}, \mathrm{CDCl}_{3}\right): \delta(\mathrm{ppm})=E$-isomer: $1.29(\mathrm{t}$, 
$J=7.1 \mathrm{~Hz}, 6 \mathrm{H}$ ), 1.67 (dddd, $J=6.8,5.7,2.8,1.4 \mathrm{~Hz}, 3 \mathrm{H}$ ), 2.51 (ddq, $J=21.4,7.4$, $1.3 \mathrm{~Hz}, 2 \mathrm{H}), 3.98-4.16(\mathrm{~m}, 4 \mathrm{H}), 5.39(\mathrm{~m}, 1 \mathrm{H}), 5.59(\mathrm{~m}, 1 \mathrm{H})$; Z-isomer: $1.29(\mathrm{t}$, $J=7.1 \mathrm{~Hz}, 6 \mathrm{H}$ ), 1.63 (dddd, $J=7.6,4.7,1.9,1.0 \mathrm{~Hz}, 3 \mathrm{H}), 2.55-2.63(\mathrm{~m}, 2 \mathrm{H}$ ), 4.01-4.14 (m, $4 \mathrm{H}), 5.42(\mathrm{~m}, 1 \mathrm{H}), 5.68(\mathrm{~m}, 1 \mathrm{H}) ;{ }^{13} \mathrm{C}$-NMR $\left(126 \mathrm{MHz}, \mathrm{CDCl}_{3}\right): \delta$ $(\mathrm{ppm})=16.4(\mathrm{~d}, J=6.0 \mathrm{~Hz}), 18.0(\mathrm{~d}, J=2.6 \mathrm{~Hz}), 30.4(\mathrm{~d}, J=139.9 \mathrm{~Hz}), 61.8(\mathrm{~d}$, $J=6.7 \mathrm{~Hz}$ ), 119.5 (d, $J=11.3 \mathrm{~Hz}), 130.7(\mathrm{~d}, J=14.8 \mathrm{~Hz}) ; \mathbf{H R}-\mathbf{M S}(\mathrm{ESI}):\left[\mathrm{C}_{8} \mathrm{H}_{18} \mathrm{O}_{3} \mathrm{P}\right]^{+}$ $\left([\mathrm{M}+\mathrm{H}]^{+}\right)$: obs.: 193.0985; calcd.: 193.0988.

\section{Diphenyl (E)-but-2-en-1-ylphosphonate (1g)}

${ }_{\mathrm{Me}} \overbrace{\mathrm{PO}(\mathrm{OPh})_{2}}$ To a solution of diphenyl phosphonate $(1.06 \mathrm{~mL}, 1.29 \mathrm{~g}, 5.50 \mathrm{mmol}$, 1.1 equiv.) in dry THF $(20 \mathrm{~mL})$ under an argon atmosphere is added $n$-butyl lithium ( $2.0 \mathrm{M}, 2.75 \mathrm{~mL}, 352 \mathrm{mg}, 5.50 \mathrm{mmol}, 1.1$ equiv.) dropwise at $-10{ }^{\circ} \mathrm{C}$ and the mixture is stirred for $10 \mathrm{~min}$. A solution of crotyl bromide (85\% trans, $514 \mu \mathrm{L}$, $675 \mathrm{mg}, 5.00 \mathrm{mmol}, 1.0$ equiv. ) in dry THF (2 mL) is added dropwise and the reaction mixture is stirred at $-10^{\circ} \mathrm{C}$ for 30 min. Sat. aqueous $\mathrm{NH}_{4} \mathrm{Cl}$-solution $(30 \mathrm{~mL})$ is added and the mixture is extracted with DCM $(3 \times 50 \mathrm{~mL})$. The combined organic layers are washed with brine $(50 \mathrm{~mL})$ and dried over $\mathrm{Na}_{2} \mathrm{SO}_{4}$. The solid is filtered off and the solvents are removed under reduced pressure. The residue is purified by column chromatography on silica gel using $n$-pentane/ $\mathrm{Et}_{2} \mathrm{O}(2: 1)$ as the eluent. The desired compound is obtained as a colourless liquid (538 mg, $1.87 \mathrm{mmol}, 37 \%, 85: 15 \mathrm{E} / \mathrm{Z}$ ).

DC: $R_{f}=0.11$ ( $n$-pentane/Et ${ }_{2} \mathrm{O}, 2: 1$ ); IR (neat): $v=3030,1590,1487,1272,1212$, 1186, 1160, 918, 757, 687, 574, $490 \mathrm{~cm}^{-1} ;{ }^{1} \mathrm{H}-\mathrm{NMR} \quad\left(300 \mathrm{MHz}, \mathrm{CDCl}_{3}\right): \delta$ $(\mathrm{ppm})=$ E-isomer: 1.67-1.75 (m, $3 \mathrm{H}), 2.78-2.92(\mathrm{~m}, 2 \mathrm{H}), 5.43-5.74(\mathrm{~m}, 2 \mathrm{H}), 7.12-$ $7.20(\mathrm{~m}, 6 \mathrm{H}), 7.25-7.34(\mathrm{~m}, 4 \mathrm{H})$; Z-isomer: 1.61-1.67 (m, $3 \mathrm{H}), 2.86-2.99(\mathrm{~m}, 2 \mathrm{H})$, 5.60-5.86 (m, $2 \mathrm{H})$, 7.09-7.17 (m, $6 \mathrm{H}), 7.23-7.31(\mathrm{~m}, 4 \mathrm{H}) ;{ }^{13} \mathrm{C}-\mathrm{NMR}(75 \mathrm{MHz}$, $\left.\mathrm{CDCl}_{3}\right): \delta(\mathrm{ppm})=E$-isomer: $18.0(\mathrm{~d}, J=2.8 \mathrm{~Hz}), 30.4(\mathrm{~d}, J=140.1 \mathrm{~Hz}), 118.2(\mathrm{~d}$, $J=11.8 \mathrm{~Hz}$ ), $120.5(\mathrm{~d}, J=4.4 \mathrm{~Hz}), 125.0(\mathrm{~d}, J=1.3 \mathrm{~Hz}), 129.6(\mathrm{~d}, J=1.1 \mathrm{~Hz}), 132.1$ (d, $J=15.5 \mathrm{~Hz}), 150.3(\mathrm{~d}, J=8.9 \mathrm{~Hz})$; Z-isomer: $12.9(\mathrm{~d}, J=2.8 \mathrm{~Hz}), 25.4(\mathrm{~d}$, $J=140.9 \mathrm{~Hz}$ ), 117.5 (d, J=11.7 Hz), 120.5 (d, $J=5.3 \mathrm{~Hz}), 125.0,129.6,129.9$ (d, $J=15.2 \mathrm{~Hz}), 150.3(\mathrm{~d}, J=9.0 \mathrm{~Hz})$; HR-MS (ESI): $\left[\mathrm{C}_{16} \mathrm{H}_{18} \mathrm{O}_{3} \mathrm{P}\right]^{+}\left([\mathrm{M}+\mathrm{H}]^{+}\right)$: obs.: 289.0991; calcd.: 289.0988 . 


\section{(Cinnamylsulfonyl)benzene (1h)}

$\mathrm{Ph}-\mathrm{SO}_{2} \mathrm{Ph}$ To a solution of cinnamyl bromide $(1.18 \mathrm{~g}, 6.00 \mathrm{mmol}, 1.0$ equiv. $)$ in $\mathrm{THF} / \mathrm{H}_{2} \mathrm{O}(10: 1,22 \mathrm{~mL})$ is added sodium benzenesulfinate $(1.48 \mathrm{~g}$, $9.00 \mathrm{mmol}, 1.5$ equiv.) and the resulting mixture is stirred at ambient temperature for $16 \mathrm{~h}$. The solution is diluted with EtOAc $(50 \mathrm{~mL})$ and $\mathrm{H}_{2} \mathrm{O}(50 \mathrm{~mL})$ and the phases are separated. The organic layer is washed with $\mathrm{H}_{2} \mathrm{O}(50 \mathrm{~mL})$ and the aqueous layers are back extracted with EtOAc $(50 \mathrm{~mL})$. The combined organic layers are dried over $\mathrm{Na}_{2} \mathrm{SO}_{4}$, the solid is filtered off and the solvents are removed under reduced pressure. The residue is purified by column chromatography on silica gel using $n$ pentane/EtOAc $(5: 1)$ as the eluent. The desired compound is obtained as a colourless oil $(1.10 \mathrm{~g}, 4.26 \mathrm{mmol}, 71 \%)$.

DC: $R_{f}=0.11$ ( $n$-pentane/EtOAc, 5:1); IR (neat): $v=2974,2904,1318,1291,1158$, 1132, 1083, 980, 906, 735, 688, 563, 528, $493 \mathrm{~cm}^{-1} ;{ }^{1} \mathrm{H}-\mathrm{NMR}\left(500 \mathrm{MHz}, \mathrm{CDCl}_{3}\right): \delta$ $($ ppm) $=3.93(\mathrm{dd}, J=7.6,1.2 \mathrm{~Hz}, 2 \mathrm{H}), 6.08(\mathrm{dt}, J=15.7,7.6 \mathrm{~Hz}, 1 \mathrm{H}), 6.35$ (dt, $J=15.7,1.2 \mathrm{~Hz}, 1 \mathrm{H}), 7.19-7.35(\mathrm{~m}, 5 \mathrm{H}), 7.47-7.58(\mathrm{~m}, 2 \mathrm{H}), 7.62(\mathrm{~m}, 1 \mathrm{H})$, 7.81-7.94 (m, $2 \mathrm{H}) ;{ }^{13} \mathrm{C}$-NMR $\left(126 \mathrm{MHz}, \mathrm{CDCl}_{3}\right): \delta(\mathrm{ppm})=60.5,115.1,126.6,128.5$, 128.6, 129.1, 133.8, 135.7, 138.3, 139.2; HR-MS (ESI): $\left[\mathrm{C}_{15} \mathrm{H}_{14} \mathrm{NaO}_{2} \mathrm{~S}\right]^{+}\left([\mathrm{M}+\mathrm{Na}]^{+}\right)$: obs.: 281.0608; calcd.: 281.0607 .

\section{(E)-(But-2-en-1-ylsulfonyl)benzene (1i)}

Me $\widehat{1 i}_{\mathrm{SO}_{2} \mathrm{Ph}}$ To a solution of crotyl bromide (85\% trans, $534 \mu \mathrm{L}, \quad 700 \mathrm{mg}$, $5.19 \mathrm{mmol}, 1.0$ equiv.) in $\mathrm{THF} / \mathrm{H}_{2} \mathrm{O}(10: 1,22 \mathrm{~mL})$ is added sodium benzenesulfinate $(1.28 \mathrm{~g}, 7.78 \mathrm{mmol}, 1.5$ equiv.) and the resulting mixture is stirred at ambient temperature for $16 \mathrm{~h}$. The solution is diluted with EtOAc $(50 \mathrm{~mL})$ and $\mathrm{H}_{2} \mathrm{O}$ $(50 \mathrm{~mL})$ and the phases are separated. The organic layer is washed with $\mathrm{H}_{2} \mathrm{O}$ $(50 \mathrm{~mL})$ and the aqueous layers are back extracted with EtOAc $(50 \mathrm{~mL})$. The combined organic layers are dried over $\mathrm{Na}_{2} \mathrm{SO}_{4}$, the solid is filtered off and the solvents are removed under reduced pressure. The residue is purified by column chromatography on silica gel using $n$-pentane/EtOAc (5:1) as the eluent. The desired compound is obtained as a colourless oil ( $798 \mathrm{mg}, 4.07 \mathrm{mmol}, 78 \%, 85: 15 \mathrm{E} / \mathrm{Z})$.

DC: $R_{f}=0.13$ ( $n$-pentane/EtOAc, 5:1); IR (neat): $v=2917,1446,1296,1138,1084$, 965, 761, 728, 687, 593, $533 \mathrm{~cm}^{-1} ;{ }^{1} \mathrm{H}$-NMR $\left(500 \mathrm{MHz}, \mathrm{CDCl}_{3}\right): \delta(\mathrm{ppm})=E$-isomer: 1.65 (dd, $J=6.5,1.5 \mathrm{~Hz}, 3 \mathrm{H}$ ), $3.71(\mathrm{~d}, J=7.3 \mathrm{~Hz}, 2 \mathrm{H}$ ), 5.40 (dtq, $J=14.8,7.2$, 
$1.7 \mathrm{~Hz}, 1 \mathrm{H}), 5.54$ (dqt, $J=15.1,6.4,1.1 \mathrm{~Hz}, 1 \mathrm{H}), 7.46-7.57(\mathrm{~m}, 2 \mathrm{H}), 7.62(\mathrm{~m}, 1 \mathrm{H})$, 7.81-7.86 (m, $2 \mathrm{H})$; Z-isomer: $1.32(\mathrm{dd}, J=7.0,1.9 \mathrm{~Hz}, 3 \mathrm{H}), 3.83(\mathrm{~d}, J=8.0 \mathrm{~Hz}$, $2 \mathrm{H}), 5.41(\mathrm{~m}, 1 \mathrm{H}), 5.80(\mathrm{~m}, 1 \mathrm{H})$, 7.50-7.56 (m, $2 \mathrm{H}), 7.61(\mathrm{~m}, 1 \mathrm{H})$, 7.85-7.89 (m, $2 \mathrm{H}) ;{ }^{13} \mathrm{C}-\mathrm{NMR}\left(126 \mathrm{MHz}, \mathrm{CDCl}_{3}\right): \delta(\mathrm{ppm})=18.1,60.1,116.9,128.4,129.0,133.6$, 136.5; HR-MS (ESI): $\left[\mathrm{C}_{10} \mathrm{H}_{12} \mathrm{NaO}_{2} \mathrm{~S}\right]^{+}\left([\mathrm{M}+\mathrm{Na}]^{+}\right)$: obs.: 219.0446; calcd.: 219.0450 .

\section{Synthesis and analytical data of acyloxylation products}

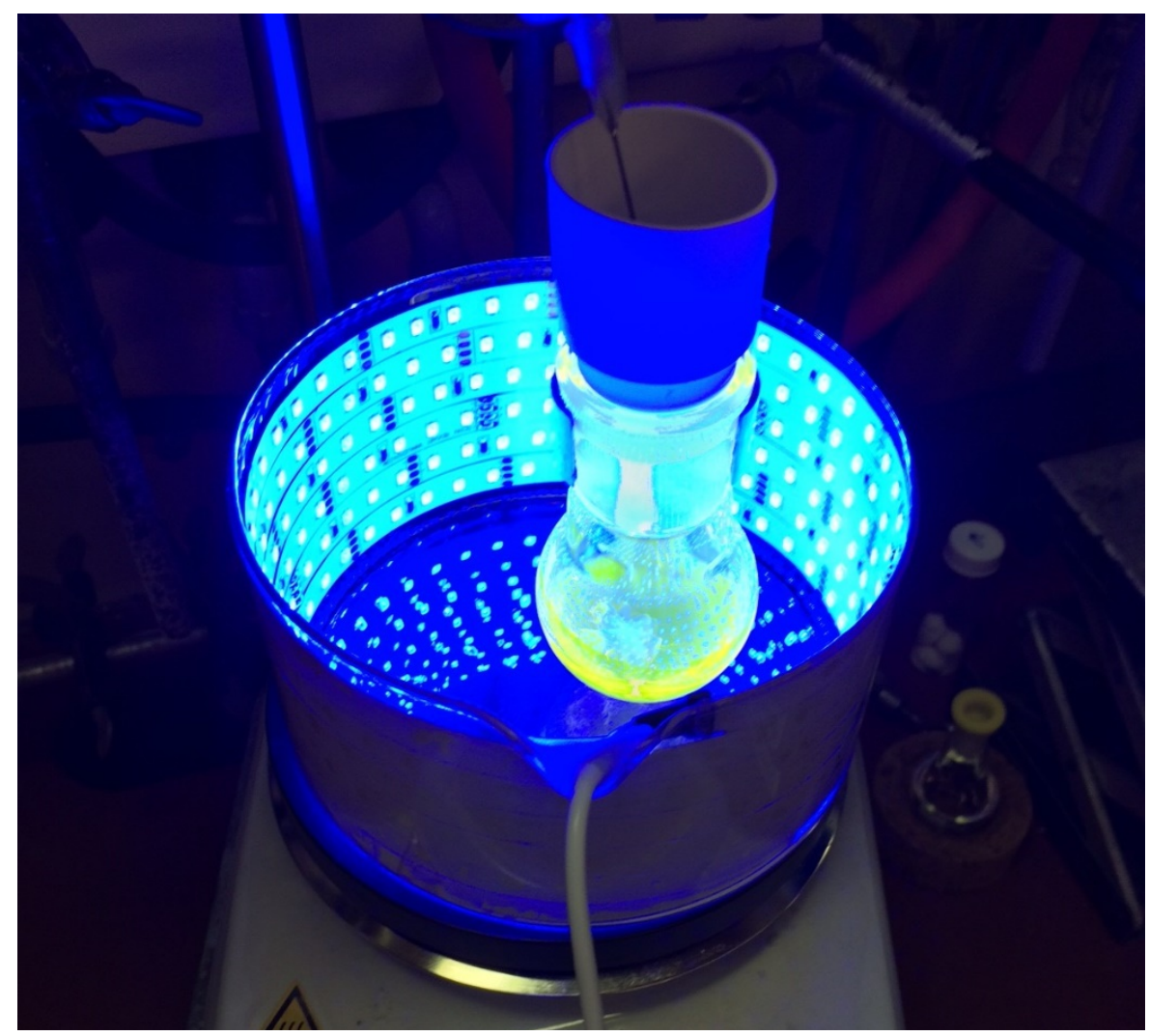

Figure S1: Reaction setup for irradiation experiments.

General procedure: To a solution of the alkene $(1.00 \mathrm{mmol}, 1.0$ equiv. $)$ in MeCN/acid $(3: 2,5 \mathrm{~mL})$ are added $(\mathrm{PhSe})_{2}(31.2 \mathrm{mg}, 100 \mu \mathrm{mol}, 0.1$ equiv.) and ( $p$ anisyl) $)_{3}$ Pyr (24.3 mg, $50.0 \mu \mathrm{mol}, 0.05$ equiv.). The mixture is subjected to irradiation at $\lambda=465 \mathrm{~nm}$ and is vigorously stirred using cross-shaped stir bars (750 rpm) under ambient air until complete conversion could be confirmed by TLC. DCM $(50 \mathrm{~mL})$ is added and the solution is washed with sat. aqueous $\mathrm{NaHCO}_{3}$-solution $(2 \times 50 \mathrm{~mL})$. The aqueous layer is back extracted with DCM $(2 \times 50 \mathrm{~mL})$. The combined organic layers are dried over $\mathrm{Na}_{2} \mathrm{SO}_{4}$, the solid is filtered off and the solvents are removed under reduced pressure. The residue is purified by column chromatography on silica gel using $\mathrm{Et}_{2} \mathrm{O} / n$-pentane as the eluent. 


\section{Benzyl (E)-4-acetoxyhex-2-enoate (3a)}

OAc Following the general procedure: benzyl (E)-hex-3-enoate (1a) (204 mg, $1.00 \mathrm{mmol}, 1.0$ equiv. $), \quad(P h S e)_{2} \quad(31.2 \mathrm{mg}, 100 \mu \mathrm{mol}$, 0.1 equiv.), ( $p$-anisyl) $)_{3}$ Pyr ( $24.3 \mathrm{mg}, 50.0 \mu \mathrm{mol}, 0.05$ equiv. $), \mathrm{MeCN}$ $(3 \mathrm{~mL}), \mathrm{AcOH}(2 \mathrm{~mL}), 16 \mathrm{~h}$; eluting with $n$-pentane/Et $\mathrm{E}_{2} \mathrm{O}, 10: 1$; yield: $234 \mathrm{mg}$, $892 \mu \mathrm{mol}, 89 \%$, colourless liquid.

DC: $R_{f}=0.23$ (n-pentane/Et $\left.2 \mathrm{O}, 10: 1\right)$; IR (neat): $v=2971,1720,1662,1373,1229$, 1164, 1016, 978, 750, $696 \mathrm{~cm}^{-1}$; ${ }^{1} \mathrm{H}-\mathrm{NMR}\left(300 \mathrm{MHz}, \mathrm{CDCl}_{3}\right): \delta(\mathrm{ppm})=0.91(\mathrm{t}$, $J=7.4 \mathrm{~Hz}, 3 \mathrm{H}), 1.62-1.75(\mathrm{~m}, 2 \mathrm{H}), 2.08(\mathrm{~s}, 3 \mathrm{H}), 5.17(\mathrm{~s}, 2 \mathrm{H}), 5.33$ (tdd, $J=6.7$, 5.3, $1.6 \mathrm{~Hz}, 1 \mathrm{H}), 5.97$ (dd, $J=15.8,1.6 \mathrm{~Hz}, 1 \mathrm{H}), 6.87$ (dd, J=15.8, $5.3 \mathrm{~Hz}, 1 \mathrm{H}$ ), 7.20-7.44 (m, $5 \mathrm{H}) ;{ }^{13} \mathrm{C}-\mathrm{NMR}\left(126 \mathrm{MHz}, \mathrm{CDCl}_{3}\right): \delta(\mathrm{ppm})=9.3,21.0,26.9,66.4,73.5$, 121.3, 128.0, 128.2, 128.5, 135.7, 145.7, 165.7, 169.9; HR-MS (ESI): $\left[\mathrm{C}_{15} \mathrm{H}_{18} \mathrm{NaO}_{4}\right]^{+}$ $\left([\mathrm{M}+\mathrm{Na}]^{+}\right):$obs.: 285.1100; calcd.: 285.1097.

\section{Phenyl (E)-4-acetoxypent-2-enoate (3b)}

Following the general procedure: phenyl $(E)$-pent-3-enoate (1c)
$(176 \mathrm{mg}, 1.00 \mathrm{mmol}, 1.0$ equiv. $), \quad(\mathrm{PhSe})_{2} \quad(31.2 \mathrm{mg}, 100 \mu \mathrm{mol}$,
0.1 equiv. $),(p \text {-anisyl })_{3} \mathrm{Pyr}(24.3 \mathrm{mg}, 50.0 \mu \mathrm{mol}, 0.05$ equiv. $), \mathrm{MeCN}$ $(3 \mathrm{~mL}), \mathrm{AcOH}(2 \mathrm{~mL}), 16 \mathrm{~h}$; eluting with $n$-pentane/Et $\mathrm{E}_{2} \mathrm{O}, 10: 1$; yield: $191 \mathrm{mg}$, $817 \mu \mathrm{mol}, 82 \%$, colourless liquid.

DC: $R_{f}=0.17$ (n-pentane/Et $\left.2 \mathrm{O}, 10: 1\right)$; IR (neat): $v=2985,1734,1660,1593,1492$, 1372, 1230, 1193, 1136, 1044, 962, 751, $688 \mathrm{~cm}^{-1} ;{ }^{1} \mathrm{H}-\mathrm{NMR}\left(400 \mathrm{MHz}, \mathrm{CDCl}_{3}\right): \delta$ $(p p m)=1.41(\mathrm{~d}, J=6.7 \mathrm{~Hz}, 3 \mathrm{H}), 2.11(\mathrm{~s}, 3 \mathrm{H}), 5.55$ (qdd, $J=6.6,4.8,1.7 \mathrm{~Hz}, 1 \mathrm{H})$, $6.15(\mathrm{dd}, J=15.7,1.7 \mathrm{~Hz}, 1 \mathrm{H}), 7.01-7.13(\mathrm{~m}, 3 \mathrm{H}), 7.24(\mathrm{~m}, 1 \mathrm{H}), 7.32-7.41(\mathrm{~m}, 2 \mathrm{H})$; ${ }^{13} \mathrm{C}-\mathrm{NMR}\left(101 \mathrm{MHz}, \mathrm{CDCl}_{3}\right): \delta(\mathrm{ppm})=19.6,21.1,68.8,120.2,121.5,125.9,129.4$, 148.4, 150.6, 164.4, 170.0; HR-MS (ESI): $\left[\mathrm{C}_{13} \mathrm{H}_{14} \mathrm{NaO}_{4}\right]^{+}\left([\mathrm{M}+\mathrm{Na}]^{+}\right)$: obs.: 257.0786; calcd.: 257.0784 .

\section{Hexyl (E)-4-acetoxyhex-2-enoate (3c)}

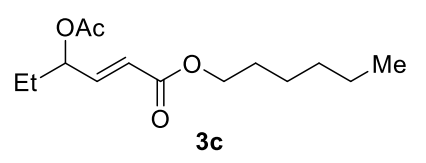

Following the general procedure: hexyl $(E)$-hex-3-enoate (1d) (252 mg, $1.00 \mathrm{mmol}, \quad 1.0$ equiv.), (PhSe) $)_{2} \quad(31.2 \mathrm{mg}$, $100 \mu \mathrm{mol}, 0.1$ equiv. $), \quad\left(p\right.$-anisyl) ${ }_{3} \mathrm{Pyr}(24.3 \mathrm{mg}, 50.0 \mu \mathrm{mol}$, 
0.05 equiv.), $\mathrm{MeCN}(3 \mathrm{~mL}), \mathrm{AcOH}(2 \mathrm{~mL}), 24 \mathrm{~h}$; eluting with $n$-pentane/Et ${ }_{2} \mathrm{O}, 10: 1$; yield: $177 \mathrm{mg}, 690 \mu \mathrm{mol}, 69 \%$, colourless liquid.

DC: $R_{f}=0.28$ (n-pentane/Et $\left.2 \mathrm{O}, 10: 1\right)$; IR (neat): $v=2933,2860,1744,1721,1663$, 1460, 1372, 1228, 1171, 1018, $978 \mathrm{~cm}^{-1} ;{ }^{1} \mathrm{H}-\mathrm{NMR}\left(300 \mathrm{MHz}, \mathrm{CDCl}_{3}\right): \delta(\mathrm{ppm})=0.74-$ $0.99(\mathrm{~m}, 6 \mathrm{H}), 1.16-1.44(\mathrm{~m}, 6 \mathrm{H}), 1.51-1.76(\mathrm{~m}, 4 \mathrm{H}), 2.07(\mathrm{~s}, 3 \mathrm{H}), 4.10(\mathrm{t}$, $J=6.7 \mathrm{~Hz}, 2 \mathrm{H}), 5.31(\mathrm{tdd}, J=6.6,5.4,1.6 \mathrm{~Hz}, 1 \mathrm{H}), 5.91(\mathrm{dd}, J=15.8,1.6 \mathrm{~Hz}, 1 \mathrm{H})$, 6.79 (dd, $J=15.8,5.4 \mathrm{~Hz}, 1 \mathrm{H}$ ); ${ }^{13} \mathrm{C}-\mathrm{NMR}\left(126 \mathrm{MHz}, \mathrm{CDCl}_{3}\right): \delta(\mathrm{ppm})=9.3,14.0$, $21.0,22.6,25.6,26.9,28.6,31.5,64.8,73.5,121.6,144.9,165.9,169.9$; HR-MS (ESI): $\left[\mathrm{C}_{14} \mathrm{H}_{24} \mathrm{NaO}_{4}\right]^{+}\left([\mathrm{M}+\mathrm{Na}]^{+}\right)$: obs.: 279.1567; calcd.: 279.1567.

\section{Benzyl (E)-4-acetoxy-4-phenylbut-2-enoate (3d)}

Following the general procedure: benzyl $(E)-4-p h e n y l b u t-3-e n o a t e ~(1 b)$
$(252 \mathrm{mg}, 1.00 \mathrm{mmol}, 1.0$ equiv. $), \quad(\mathrm{PhSe})_{2} \quad(31.2 \mathrm{mg}, 100 \mu \mathrm{mol}$,
0.1 equiv. $),(p \text {-anisyl })_{3} \mathrm{Pyr}(24.3 \mathrm{mg}, 50.0 \mu \mathrm{mol}, 0.05$ equiv. $), \mathrm{MeCN}$ $(3 \mathrm{~mL}), \mathrm{AcOH}(2 \mathrm{~mL})$, oxygen atmosphere, $16 \mathrm{~h}$; eluting with $n$-pentane/Et ${ }_{2} \mathrm{O}, 5: 1$; yield: $141 \mathrm{mg}, 453 \mu \mathrm{mol}, 45 \%$, colourless oil.

DC: $R_{f}=0.50$ (n-pentane/Et $2 \mathrm{O}, 5: 1$ ); IR (neat): $v=3033,1718,1659,1455,1371$, 1222, 1161, 1018, 977, 750, $695 \mathrm{~cm}^{-1} ;{ }^{1} \mathrm{H}-\mathrm{NMR}\left(400 \mathrm{MHz}, \mathrm{CDCl}_{3}\right): \delta(\mathrm{ppm})=2.11$ (s, $3 \mathrm{H}), 5.16(\mathrm{~d}, J=1.1 \mathrm{~Hz}, 2 \mathrm{H}), 6.07(\mathrm{dd}, J=15.7,1.8 \mathrm{~Hz}, 1 \mathrm{H}), 6.38(\mathrm{dd}, J=5.0$, $1.8 \mathrm{~Hz}, 1 \mathrm{H}), 7.04$ (dd, $J=15.7,5.0 \mathrm{~Hz}, 1 \mathrm{H}), 7.24-7.45(\mathrm{~m}, 10 \mathrm{H}) ;{ }^{13} \mathrm{C}-\mathrm{NMR}$ $\left(101 \mathrm{MHz}, \mathrm{CDCl}_{3}\right): \delta(\mathrm{ppm})=21.0,66.5,74.1,121.3,127.4,128.3,128.4,128.6$, 128.8, 128.8, 135.7, 137.0, 145.2, 165.7, 169.6; HR-MS (ESI): $\left[\mathrm{C}_{19} \mathrm{H}_{18} \mathrm{NaO}_{4}\right]^{+}$ $\left([\mathrm{M}+\mathrm{Na}]^{+}\right)$: obs.: 333.1098; calcd.: 333.1097.

\section{(E)-1-Cyanohex-1-en-3-yl acetate (3e)}

OAC Following the general procedure: $(E)$-hept-3-enenitrile (1e) $(109 \mathrm{mg}$, 3e ${ }^{\mathrm{CN}} 1.00 \mathrm{mmol}, 1.0$ equiv.), (PhSe) 2 (31.2 mg, $100 \mu \mathrm{mol}, 0.1$ equiv.), ( $p$ anisyl) $)_{3}$ Pyr (24.3 mg, $50.0 \mu \mathrm{mol}, 0.05$ equiv.), MeCN (3 mL), AcOH (2 mL), $24 \mathrm{~h}$; eluting with $n$-pentane/ $\mathrm{Et}_{2} \mathrm{O}, 20: 1$; yield: $139 \mathrm{mg}, 831 \mu \mathrm{mol}, 83 \%$, colourless liquid.

DC: $R_{f}=0.24$ (n-pentane/Et $2 \mathrm{O}, 20: 1$ ); IR (neat): $v=2963,2876,2227,1737,1373$, 1224, 1025, $966 \mathrm{~cm}^{-1}$; ${ }^{1} \mathrm{H}-\mathrm{NMR}\left(400 \mathrm{MHz} \mathrm{CDCl}_{3}\right): \delta(\mathrm{ppm})=0.90(\mathrm{t}, \mathrm{J}=7.3 \mathrm{~Hz}, 3 \mathrm{H})$, 1.20-1.46 (m, $2 \mathrm{H})$, 1.47-1.72 (m, $2 \mathrm{H}), 2.07(\mathrm{~s}, 3 \mathrm{H}), 5.34(\mathrm{~m}, 1 \mathrm{H}), 5.48$ (dd, 
$J=16.4,1.7 \mathrm{~Hz}, 1 \mathrm{H}), 6.60(\mathrm{dd}, J=16.4,5.3 \mathrm{~Hz}, 1 \mathrm{H}) ;{ }^{13} \mathrm{C}-\mathrm{NMR}\left(101 \mathrm{MHz}, \mathrm{CDCl}_{3}\right): \delta$ $(\mathrm{ppm})=13.6,18.1,20.8,35.5,72.0,100.1,116.5,151.9,169.8$; HR-MS (ESI): $\left[\mathrm{C}_{9} \mathrm{H}_{13} \mathrm{NNaO}_{2}\right]^{+}\left([\mathrm{M}+\mathrm{Na}]^{+}\right)$: obs.: 190.0841; calcd.: 190.0838 .

\section{(E)-4-(Diethoxyphosphoryl)but-3-en-2-yl acetate (3f)}

OAC Following the general procedure: diethyl (E)-but-2-en-1${ }_{3 \mathrm{fo}} \mathrm{POEt}_{2}$ ylphosphonate (1f) $(192 \mathrm{mg}, 1.00 \mathrm{mmol}, 1.0 \text { equiv.), (PhSe })_{2}$ (31.2 mg, $100 \mu \mathrm{mol}, 0.1$ equiv.), ( $p$-anisyl) $)_{3} P y r \quad(24.3 \mathrm{mg}, 50.0 \mu \mathrm{mol}, 0.05$ equiv.), $\mathrm{MeCN}(3 \mathrm{~mL}), \mathrm{AcOH}(2 \mathrm{~mL}), 16 \mathrm{~h}$; eluting with $\mathrm{Et}_{2} \mathrm{O}$; yield: $169 \mathrm{mg}, 675 \mu \mathrm{mol}, 68 \%$, colourless liquid.

DC: $R_{f}=0.17\left(\mathrm{Et}_{2} \mathrm{O}\right) ; \mathbf{R}$ (neat): $v=2983,1738,1372,1231,1018,955,810$, $522 \mathrm{~cm}^{-1} ;{ }^{1} \mathrm{H}-\mathrm{NMR}\left(300 \mathrm{MHz}, \mathrm{CDCl}_{3}\right): \delta(\mathrm{ppm})=1.11-1.41(\mathrm{~m}, 9 \mathrm{H}), 2.01(\mathrm{~s}, 3 \mathrm{H})$, 3.85-4.19 (m, $4 \mathrm{H}$ ), 5.38 (m, $1 \mathrm{H}$ ), 5.75 (dddd, $J=19.0,17.2,1.7,0.7 \mathrm{~Hz}, 1 \mathrm{H}$ ), 6.62 (dddd, $J=22.4,17.2,4.4,0.7 \mathrm{~Hz}, 1 \mathrm{H}) ;{ }^{13} \mathrm{C}-\mathrm{NMR}\left(126 \mathrm{MHz}, \mathrm{CDCl}_{3}\right): \delta(\mathrm{ppm})=16.3$ (d, $J=6.3 \mathrm{~Hz}$ ), 19.4 (d, $J=1.9 \mathrm{~Hz}), 21.0,61.8(\mathrm{~d}, J=5.6 \mathrm{~Hz}), 69.5(\mathrm{~d}, J=22.5 \mathrm{~Hz}$ ), 116.7 (d, $J=188.2 \mathrm{~Hz}$ ), 150.3 (d, $J=5.4 \mathrm{~Hz}$ ), 169.6; HR-MS (ESI): $\left[\mathrm{C}_{10} \mathrm{H}_{20} \mathrm{O}_{5} \mathrm{P}\right]^{+}$ $\left([\mathrm{M}+\mathrm{H}]^{+}\right)$: obs.: 251.1044 ; calcd.: 251.1043 .

\section{(E)-4-(Diphenoxyphosphoryl)but-3-en-2-yl acetate (3g)}

OAC Following the general procedure: diphenyl (E)-but-2-en-1${ }_{3 \mathrm{~g}}^{\mathrm{PO}(\mathrm{OPh})_{2}}$ ylphosphonate $(\mathbf{1 g}) \quad(288 \mathrm{mg}, 1.00 \mathrm{mmol}, 1.0$ equiv. $), \quad(\mathrm{PhSe})_{2}$ (31.2 mg, $100 \mu \mathrm{mol}, 0.1$ equiv.), ( $p$-anisyl) $)_{3} P y r(24.3 \mathrm{mg}, 50.0 \mu \mathrm{mol}, 0.05$ equiv.), $\mathrm{MeCN}(3 \mathrm{~mL}), \mathrm{AcOH}(2 \mathrm{~mL}), 16 \mathrm{~h}$; eluting with $n$-pentane/ $\mathrm{Et}_{2} \mathrm{O}, 1: 1$; yield: $231 \mathrm{mg}$, $668 \mu \mathrm{mol}, 67 \%$, colourless oil.

DC: $R_{f}=0.15\left(n\right.$-pentane/Et $\left.{ }_{2} \mathrm{O}, 1: 1\right) ;$ IR (neat): $v=2985,1738,1590,1487,1372$, 1271, 1210, 1185, 1161, 921, 761, 688, $511 \mathrm{~cm}^{-1} ;{ }^{1} \mathrm{H}-\mathrm{NMR}\left(300 \mathrm{MHz}, \mathrm{CDCl}_{3}\right): \delta$ $(\mathrm{ppm})=1.31(\mathrm{~d}, J=6.7 \mathrm{~Hz}, 3 \mathrm{H}), 2.06(\mathrm{~s}, 3 \mathrm{H}), 5.45(\mathrm{~m}, 1 \mathrm{H}), 6.05$ (ddd, $J=21.0$, 17.2, $1.7 \mathrm{~Hz}, 1 \mathrm{H}$ ), 6.87 (ddd, $J=23.8,17.2,4.4 \mathrm{~Hz}, 1 \mathrm{H}), 7.06-7.23(\mathrm{~m}, 6 \mathrm{H}), 7.23-$ $7.41(\mathrm{~m}, 4 \mathrm{H}) ;{ }^{13} \mathrm{C}-\mathrm{NMR}\left(75 \mathrm{MHz}, \mathrm{CDCl}_{3}\right): \delta(\mathrm{ppm})=19.3(\mathrm{~d}, J=2.0 \mathrm{~Hz}), 21.0,69.5$ (d, $J=23.5 \mathrm{~Hz}), 115.6(\mathrm{~d}, J=192.9 \mathrm{~Hz}), 120.6(\mathrm{~d}, J=4.5 \mathrm{~Hz}), 125.2(\mathrm{~d}, J=1.5 \mathrm{~Hz}$ ), 129.7, 150.1 (d, $J=7.8 \mathrm{~Hz}$ ), 153.3 (d, $J=5.8 \mathrm{~Hz}$ ), 169.7; HR-MS (ESI): $\left[\mathrm{C}_{18} \mathrm{H}_{20} \mathrm{O}_{5} \mathrm{P}\right]^{+}$ $\left([\mathrm{M}+\mathrm{H}]^{+}\right)$: obs.: 347.1050 ; calcd.: 347.1043 . 


\section{(E)-1-Phenyl-3-(phenylsulfonyl)allyl acetate (3h)}

Following the general procedure: (cinnamylsulfonyl)benzene (1h) ${ }_{3 \mathrm{~h}}^{\mathrm{SO}_{2} \mathrm{Ph}}(258 \mathrm{mg}, \quad 1.00 \mathrm{mmol}, 1.0$ equiv. $), \quad(\mathrm{PhSe})_{2} \quad(31.2 \mathrm{mg}, \quad 100 \mu \mathrm{mol}$, 0.1 equiv. $),\left(p\right.$-anisyl) ${ }_{3} P y r(24.3 \mathrm{mg}, 50.0 \mu \mathrm{mol}, 0.05$ equiv. $), \mathrm{MeCN}(3 \mathrm{~mL}), \mathrm{AcOH}$ $(2 \mathrm{~mL})$, oxygen atmosphere, $16 \mathrm{~h}$; eluting with $n$-pentane/Et ${ }_{2} \mathrm{O}, 5: 1$; yield: $192 \mathrm{mg}$, $607 \mu \mathrm{mol}, 61 \%$, colourless oil.

DC: $R_{f}=0.14$ (n-pentane/Et $\left.{ }_{2} \mathrm{O}, 5: 1\right)$; IR (neat): $v=3062,1738,1447,1371,1307$, 1222, 1144, 1084, 1021, 971, 751, 686, 592, 557, $529 \mathrm{~cm}^{-1} ;{ }^{1} \mathrm{H}-\mathrm{NMR}(300 \mathrm{MHz}$, $\left.\mathrm{CDCl}_{3}\right): \delta(\mathrm{ppm})=2.07(\mathrm{~s}, 3 \mathrm{H}), 6.41(\mathrm{dd}, J=4.3,1.8 \mathrm{~Hz}, 1 \mathrm{H}), 6.55(\mathrm{dd}, J=15.0$, $1.8 \mathrm{~Hz}, 1 \mathrm{H}), 7.03(\mathrm{dd}, J=15.0,4.3 \mathrm{~Hz}, 1 \mathrm{H}), 7.22-7.40(\mathrm{~m}, 5 \mathrm{H}), 7.46-7.69(\mathrm{~m}, 3 \mathrm{H})$, 7.78-7.91 (m, $2 \mathrm{H}) ;{ }^{13} \mathrm{C}-N M R\left(126 \mathrm{MHz} \mathrm{CDCl}_{3}\right): \delta(\mathrm{ppm})=21.0,73.2,127.4,127.7$, 128.9, 129.0, 129.3, 130.8, 133.5, 136.0, 139.8, 142.7, 169.1; HR-MS (ESI): $\left[\mathrm{C}_{17} \mathrm{H}_{16} \mathrm{NaO}_{4} \mathrm{~S}\right]^{+}\left([\mathrm{M}+\mathrm{Na}]^{+}\right):$obs.: 339.0663; calcd.: 339.0662.

\section{(E)-4-(Phenylsulfonyl)but-3-en-2-yl acetate (3i)}

OAc Following the general procedure: $(E)$-(but-2-en-1-ylsulfonyl)benzene $\widehat{\mathrm{Me}}_{\mathbf{3 i}}^{\mathrm{SO}_{2} \mathrm{Ph}}$ (1i) $(196 \mathrm{mg}, 1.00 \mathrm{mmol}, 1.0 \text { equiv.), (PhSe) })_{2}(31.2 \mathrm{mg}, 100 \mu \mathrm{mol}$, 0.1 equiv.), ( $p$-anisyl) ${ }_{3} P y r(24.3 \mathrm{mg}, 50.0 \mu \mathrm{mol}, 0.05$ equiv.), $\mathrm{MeCN}(3 \mathrm{~mL}), \mathrm{AcOH}$ $(2 \mathrm{~mL})$, oxygen atmosphere, $16 \mathrm{~h}$; eluting with $n$-pentane/ $\mathrm{Et}_{2} \mathrm{O}, 5: 1$; yield: $164 \mathrm{mg}$, $645 \mu \mathrm{mol}, 65 \%$, colourless oil.

DC: $R_{f}=0.19$ (n-pentane/Et ${ }_{2} \mathrm{O}, 5: 1$ ); IR (neat): $v=3061,1738,1307,1229,1143$, 1085, 1043, 957, 826, 753, 718, 687, 590, 574, 553, $522 \mathrm{~cm}^{-1} ;{ }^{1} \mathrm{H}-\mathrm{NMR}(300 \mathrm{MHz}$, $\left.\mathrm{CDCl}_{3}\right): \delta(\mathrm{ppm})=1.33(\mathrm{~d}, J=6.7 \mathrm{~Hz}, 3 \mathrm{H}), 2.01(\mathrm{~s}, 3 \mathrm{H}), 5.47(\mathrm{~m}, 1 \mathrm{H}), 6.45$ (dd, $J=15.1,1.7 \mathrm{~Hz}, 1 \mathrm{H}), 6.88(\mathrm{dd}, J=15.1,4.3 \mathrm{~Hz}, 1 \mathrm{H}), 7.42-7.65(\mathrm{~m}, 3 \mathrm{H}), 7.77-7.91$ $(\mathrm{m}, 2 \mathrm{H}) ;{ }^{13} \mathrm{C}-\mathbf{N M R}\left(75 \mathrm{MHz}, \mathrm{CDCl}_{3}\right): \delta(\mathrm{ppm})=19.3,20.9,67.8,127.6,129.3,130.2$, 133.5, 139.8, 144.2, 169.5; HR-MS (ESI): $\left[\mathrm{C}_{12} \mathrm{H}_{14} \mathrm{NaO}_{4} \mathrm{~S}\right]^{+}\left([\mathrm{M}+\mathrm{Na}]^{+}\right):$obs.: 277.0508; calcd.: 277.0505 .

\section{(E)-Dec-6-en-5-yl acetate (3j)}

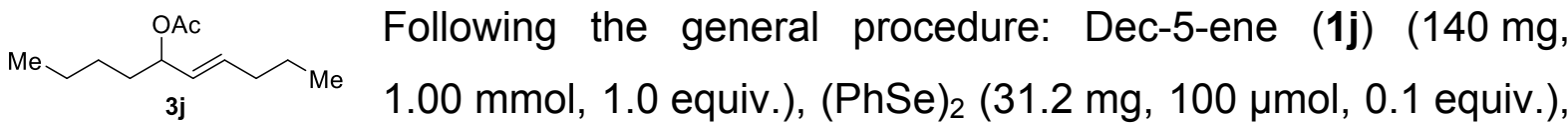
(p-anisyl) ${ }_{3}$ Pyr (24.3 mg, $50.0 \mu \mathrm{mol}, 0.05$ equiv.), MeCN (3 mL), AcOH (2 mL), $16 \mathrm{~h}$; eluting with $n$-pentane/Et ${ }_{2} \mathrm{O}, 20: 1$; yield: starting from $(E)$-dec-5-ene: $161 \mathrm{mg}$, 
$813 \mu \mathrm{mol}, 81 \%$, colourless liquid; starting from (Z)-dec-5-ene: $148 \mathrm{mg}, 748 \mu \mathrm{mol}$, $75 \%$, colourless liquid.

DC: $R_{f}=0.24$ (n-pentane/Et $2 \mathrm{O}, 20: 1$ ); IR (neat): $v=2958,2931,2863,1736,1465$, 1370, 1233, 1017, $967 \mathrm{~cm}^{-1}$; ${ }^{1} \mathrm{H}-\mathrm{NMR}\left(300 \mathrm{MHz}, \mathrm{CDCl}_{3}\right): \delta(\mathrm{ppm})=0.64-0.98(\mathrm{~m}$, $6 \mathrm{H}), 1.08-1.68(\mathrm{~m}, 9 \mathrm{H}), 1.87-2.06(\mathrm{~m}, 4 \mathrm{H}), 5.13(\mathrm{q}, J=6.9 \mathrm{~Hz}, 1 \mathrm{H}), 5.32$ (ddt, $J=15.3,7.4,1.4 \mathrm{~Hz}, 1 \mathrm{H}), 5.63$ (dtd, $J=15.3,6.7,0.8 \mathrm{~Hz}, 1 \mathrm{H}) ;{ }^{13} \mathrm{C}-\mathrm{NMR}(75 \mathrm{MHz}$, $\left.\mathrm{CDCl}_{3}\right): \delta(\mathrm{ppm})=13.5,13.9,21.3,22.0,22.4,27.3,34.2,75.0,128.5,134.0,170.3$; HR-MS (ESI): $\left[\mathrm{C}_{12} \mathrm{H}_{23} \mathrm{O}_{2}\right]^{+}\left([\mathrm{M}+\mathrm{H}]^{+}\right)$: obs.: 199.1695; calcd.: 199.1693.

\section{(Z)-Cyclooct-2-en-1-yl acetate (3k)}

OAc Following the general procedure: (Z)-cyclooctene (1k) (111 mg, $1.01 \mathrm{mmol}, 1.0$ equiv. $), \quad(\mathrm{PhSe})_{2} \quad(31.2 \mathrm{mg}, 100 \mu \mathrm{mol}, 0.1$ equiv. $), \quad(p-$ anisyl) ${ }_{3} \operatorname{Pyr}(24.3 \mathrm{mg}, 50.0 \mu \mathrm{mol}, 0.05$ equiv.), MeCN ( $3 \mathrm{~mL}), \mathrm{AcOH}(2 \mathrm{~mL}), 16 \mathrm{~h}$; eluting with $n$-pentane/Et ${ }_{2} \mathrm{O}, 20: 1$; yield: $151 \mathrm{mg}, 897 \mu \mathrm{mol}, 89 \%$, colourless liquid.

DC: $R_{f}=0.46$ (n-pentane/Et $\left.2 \mathrm{O}, 20: 1\right)$; IR (neat): $v=2928,2858,1732,1450,1370$, 1237, 1028, 960, 904, 756, $712 \mathrm{~cm}^{-1} ;{ }^{1} \mathrm{H}-\mathrm{NMR}\left(300 \mathrm{MHz}, \mathrm{CDCl}_{3}\right): \delta(\mathrm{ppm})=1.25-$ $1.75(\mathrm{~m}, 7 \mathrm{H}), 1.87(\mathrm{tdd}, J=8.0,3.8,1.7 \mathrm{~Hz}, 1 \mathrm{H}), 2.01(\mathrm{~s}, 3 \mathrm{H}), 2.01-2.35(\mathrm{~m}, 2 \mathrm{H})$, 5.44 (ddd, $J=10.8,7.0,1.3 \mathrm{~Hz}, 1 \mathrm{H}), 5.55-5.74(\mathrm{~m}, 2 \mathrm{H}) ;{ }^{13} \mathrm{C}-\mathrm{NMR}\left(75 \mathrm{MHz}, \mathrm{CDCl}_{3}\right)$ : $\delta(\mathrm{ppm})=21.4,23.3,25.8,26.3,28.7,35.1,72.3,129.7,130.6,170.4$; HR-MS (ESI): $\left[\mathrm{C}_{10} \mathrm{H}_{16} \mathrm{NaO}_{2}\right]^{+}\left([\mathrm{M}+\mathrm{Na}]^{+}\right):$obs.: 191.1042; calcd.: 191.1043.

(E)-3-(4-(Trifluoromethyl)phenyl)allyl acetate (3I)

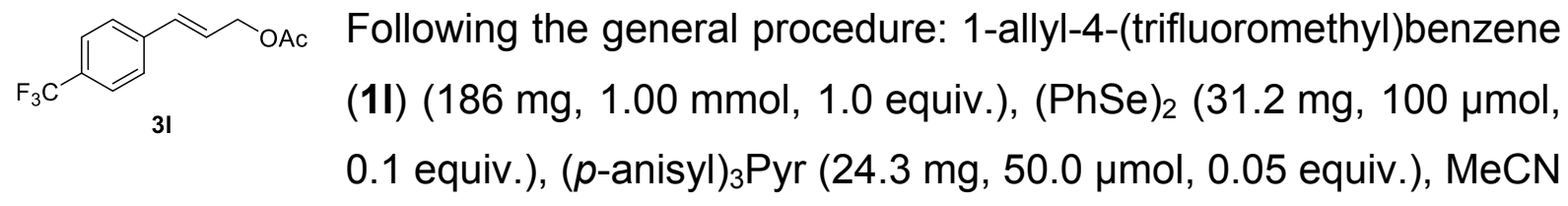
$(3 \mathrm{~mL}), \mathrm{AcOH}(2 \mathrm{~mL}), 16 \mathrm{~h}$; eluting with $n$-pentane/Et ${ }_{2} \mathrm{O}, 10: 1$; yield: $76.0 \mathrm{mg}$, $311 \mu \mathrm{mol}, 31 \%$, colourless liquid; contains $9 \%$ of cyclized indane byproduct.

DC: $R_{f}=0.21$ (n-pentane/Et $2 \mathrm{O}, 10: 1$ ); IR (neat): $v=2937,1737,1322,1225,1162$, 1115, 1065, 1016, 967, 854, 738, $596 \mathrm{~cm}^{-1} ;{ }^{1} \mathrm{H}-\mathrm{NMR} \quad\left(500 \mathrm{MHz}, \mathrm{CDCl}_{3}\right): \delta$ $(\mathrm{ppm})=2.10(\mathrm{~s}, 3 \mathrm{H}), 4.73(\mathrm{dd}, J=6.3,1.5 \mathrm{~Hz}, 2 \mathrm{H}), 6.36(\mathrm{dt}, J=15.9,6.2 \mathrm{~Hz}, 1 \mathrm{H})$, $6.66(\mathrm{dt}, J=16.0,1.5 \mathrm{~Hz}, 1 \mathrm{H}), 7.46(\mathrm{~d}, J=8.3 \mathrm{~Hz}, 2 \mathrm{H}), 7.56(\mathrm{~d}, J=8.3 \mathrm{~Hz}, 2 \mathrm{H})$; ${ }^{13} \mathrm{C}-N M R\left(126 \mathrm{MHz}, \mathrm{CDCl}_{3}\right): \delta(\mathrm{ppm})=20.9,64.6,124.1$ (q, $\left.J=271.9 \mathrm{~Hz}\right), 125.6$ (q, 
$J=3.8 \mathrm{~Hz}$ ), 126.0, 126.7, 129.2, 129.8, 129.8 (q, $J=32.5 \mathrm{~Hz}), 132.3,132.9,139.6$, 170.7; HR-MS (ESI): $\left[\mathrm{C}_{12} \mathrm{H}_{11} \mathrm{~F}_{3} \mathrm{NaO}_{2}\right]^{+}\left([\mathrm{M}+\mathrm{Na}]^{+}\right)$: obs.: 267.0598; calcd.: 267.0603.

\section{Benzyl (E)-4-(formyloxy)hex-2-enoate (3m)}

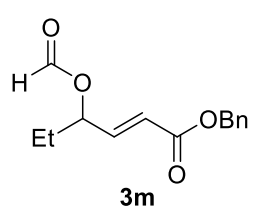

Following the general procedure: benzyl (E)-hex-3-enoate (1a) (204 mg, $1.00 \mathrm{mmol}, 1.0$ equiv.), (PhSe) $2 \quad(31.2 \mathrm{mg}, 100 \mu \mathrm{mol}$, 0.1 equiv.), ( $p$-anisyl $)_{3}$ Pyr ( $24.3 \mathrm{mg}, 50.0 \mu \mathrm{mol}, 0.05$ equiv.), $\mathrm{MeCN}$ $(3 \mathrm{~mL})$, formic acid $(2 \mathrm{~mL}), 24 \mathrm{~h}$; eluting with $n$-pentane/Et $\mathrm{t}_{2} \mathrm{O}, 5: 1$; yield: $201 \mathrm{mg}$, $810 \mu \mathrm{mol}, 81 \%$, colourless liquid.

DC: $R_{f}=0.22\left(n\right.$-pentane/Et $\left.{ }_{2} \mathrm{O}, 5: 1\right)$; IR (neat): $v=2972,1716,1662,1456,1378$, 1270, 1154, 977, 739, $696 \mathrm{~cm}^{-1} ;{ }^{1} \mathrm{H}-\mathrm{NMR}\left(400 \mathrm{MHz}, \mathrm{CDCl}_{3}\right): \delta(\mathrm{ppm})=0.93(\mathrm{t}$, $J=7.4 \mathrm{~Hz}, 3 \mathrm{H}), 1.62-1.79(\mathrm{~m}, 2 \mathrm{H}), 5.17(\mathrm{~s}, 2 \mathrm{H}), 5.46$ (tdd, $J=6.9,5.3,1.3 \mathrm{~Hz}$, $1 \mathrm{H}), 6.02(\mathrm{dd}, J=15.8,1.6 \mathrm{~Hz}, 1 \mathrm{H}$ ), 6.87 (dd, $J=15.8,5.3 \mathrm{~Hz}, 1 \mathrm{H}), 7.28-7.45(\mathrm{~m}$, $5 \mathrm{H}), 8.08(\mathrm{~s}, 1 \mathrm{H}) ;{ }^{13} \mathrm{C}-\mathrm{NMR}\left(101 \mathrm{MHz}, \mathrm{CDCl}_{3}\right): \delta(\mathrm{ppm})=9.2,26.8,66.5,73.3$, 121.9, 128.3, 128.3, 128.6, 135.7, 144.8, 160.0, 165.6; HR-MS (ESI): $\left[\mathrm{C}_{14} \mathrm{H}_{16} \mathrm{NaO}_{4}\right]^{+}$ $\left([\mathrm{M}+\mathrm{Na}]^{+}\right)$: obs.: 271.0943 ; calcd.: 271.0941 .

\section{Benzyl (E)-4-(propionyloxy)hex-2-enoate (3n)}

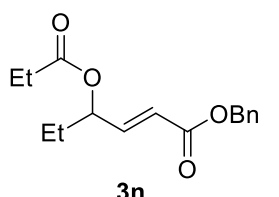

Following the general procedure: benzyl (E)-hex-3-enoate (1a) (204 mg, $1.00 \mathrm{mmol}, 1.0$ equiv.), (PhSe) ${ }_{2} \quad(31.2 \mathrm{mg}, 100 \mu \mathrm{mol}$, 0.1 equiv.), ( $p$-anisyl) $)_{3} P y r(24.3 \mathrm{mg}, 50.0 \mu \mathrm{mol}, 0.05$ equiv.), $\mathrm{MeCN}$ $(3 \mathrm{~mL})$, propionic acid $(2 \mathrm{~mL}), 24 \mathrm{~h}$; eluting with $n$-pentane/ $\mathrm{Et}_{2} \mathrm{O}, 5: 1$; yield: $173 \mathrm{mg}$, $626 \mu \mathrm{mol}, 63 \%$, colourless liquid.

DC: $R_{f}=0.24$ (n-pentane/Et $\left.{ }_{2} \mathrm{O}, 5: 1\right)$; IR (neat): $v=2974,1720,1662,1457,1378$, 1269, 1160, 1080, 978, 739, $697 \mathrm{~cm}^{-1} ;{ }^{1} \mathrm{H}-\mathrm{NMR}\left(400 \mathrm{MHz}, \mathrm{CDCl}_{3}\right): \delta(\mathrm{ppm})=0.91(\mathrm{t}$, $J=7.5 \mathrm{~Hz}, 3 \mathrm{H}$ ), $1.14(\mathrm{t}, J=7.6 \mathrm{~Hz}, 3 \mathrm{H}), 1.60-1.76(\mathrm{~m}, 2 \mathrm{H}), 2.36(\mathrm{q}, J=7.5 \mathrm{~Hz}$, $2 \mathrm{H}$ ), 5.17 (s, $2 \mathrm{H}$ ), 5.35 (dddd, $J=6.8,6.0,5.2,1.6 \mathrm{~Hz}, 1 \mathrm{H}$ ), 5.97 (dd, $J=15.8$, $1.6 \mathrm{~Hz}, \quad 1 \mathrm{H}), \quad 6.88(\mathrm{dd}, J=15.8, \quad 5.2 \mathrm{~Hz}, 1 \mathrm{H}), 7.27-7.41(\mathrm{~m}, 5 \mathrm{H}) ;{ }^{13} \mathrm{C}-\mathrm{NMR}$ $\left(101 \mathrm{MHz}, \mathrm{CDCl}_{3}\right): \delta(\mathrm{ppm})=9.1,9.2,26.9,27.6,66.4,73.2,121.2,128.3,128.3$, 128.5, 135.8, 146.0, 165.8, 173.5; HR-MS (ESI): $\left[\mathrm{C}_{16} \mathrm{H}_{20} \mathrm{NaO}_{4}\right]^{+}\left([\mathrm{M}+\mathrm{Na}]^{+}\right)$: obs.: 299.1258; calcd.: 299.1254 . 


\section{Benzyl (E)-4-(butyryloxy)hex-2-enoate (3o)}

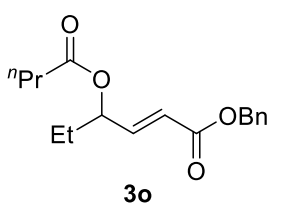

Following the general procedure: benzyl (E)-hex-3-enoate (1a) (204 mg, $1.00 \mathrm{mmol}, 1.0$ equiv.), (PhSe) ${ }_{2} \quad(31.2 \mathrm{mg}, 100 \mu \mathrm{mol}$, 0.1 equiv.), ( $p$-anisyl) ${ }_{3} P y r(24.3 \mathrm{mg}, 50.0 \mu \mathrm{mol}, 0.05$ equiv.), $\mathrm{MeCN}$ $(3 \mathrm{~mL})$, butyric acid $(2 \mathrm{~mL}), 24 \mathrm{~h}$; eluting with $n$-pentane/ $\mathrm{Et}_{2} \mathrm{O}, 5: 1$; yield: $158 \mathrm{mg}$, $545 \mu \mathrm{mol}, 55 \%$, colourless liquid.

DC: $R_{f}=0.22$ (n-pentane/Et $\left.{ }_{2} \mathrm{O}, 5: 1\right)$; IR (neat): $v=2968,2878,1721,1661,1456$, 1380, 1269, 1160, 977, 741, $696 \mathrm{~cm}^{-1}$; ${ }^{1} \mathrm{H}-\mathrm{NMR}\left(400 \mathrm{MHz}, \mathrm{CDCl}_{3}\right): \delta(\mathrm{ppm})=0.90(\mathrm{t}$, $J=7.4 \mathrm{~Hz}, 3 \mathrm{H}), 0.94(\mathrm{t}, J=7.4 \mathrm{~Hz}, 3 \mathrm{H}), 1.59-1.75(\mathrm{~m}, 4 \mathrm{H}), 2.27-2.35(\mathrm{~m}, 2 \mathrm{H}), 5.16$ $(\mathrm{d}, J=12.4 \mathrm{~Hz}, 1 \mathrm{H}$ ), 5.17 (d, $J=12.4 \mathrm{~Hz}, 1 \mathrm{H}$ ), 5.35 (dddd, $J=6.8,6.0,5.1,1.6 \mathrm{~Hz}$, $1 \mathrm{H}$ ), 5.97 (dd, $J=15.7,1.6 \mathrm{~Hz}, 1 \mathrm{H}$ ), $6.88(\mathrm{dd}, J=15.7,5.2 \mathrm{~Hz}, 1 \mathrm{H}), 7.26-7.43(\mathrm{~m}$, $5 \mathrm{H}) ;{ }^{13} \mathrm{C}-\mathrm{NMR}\left(101 \mathrm{MHz}, \mathrm{CDCl}_{3}\right): \delta(\mathrm{ppm})=9.2,13.6,18.4,26.9,36.2,66.4,73.2$, 121.2, 128.3, 128.3, 128.6, 135.8, 146.0, 165.8, 172.7; HR-MS (ESI): $\left[\mathrm{C}_{17} \mathrm{H}_{22} \mathrm{NaO}_{4}\right]^{+}$ $\left([\mathrm{M}+\mathrm{Na}]^{+}\right):$obs.: 313.1411 ; calcd.: 313.1410 .

\section{Benzyl (E)-4-(isobutyryloxy)hex-2-enoate (3p)}

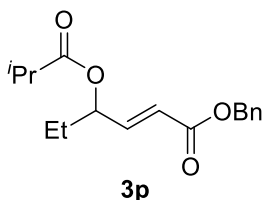

$3 p$

Following the general procedure: benzyl (E)-hex-3-enoate (1a) (204 mg, $1.00 \mathrm{mmol}, 1.0$ equiv.), (PhSe) $2 \quad(31.2 \mathrm{mg}, 100 \mu \mathrm{mol}$, 0.1 equiv.), ( $p$-anisyl) ${ }_{3} P y r(24.3 \mathrm{mg}, 50.0 \mu \mathrm{mol}, 0.05$ equiv.), $\mathrm{MeCN}$ $(3 \mathrm{~mL})$, isobutyric acid $(2 \mathrm{~mL}), 48 \mathrm{~h}$; eluting with $n$-pentane/ $\mathrm{Et}_{2} \mathrm{O}, 10: 1$; yield: $157 \mathrm{mg}$, $540 \mu \mathrm{mol}, 54 \%$, colourless liquid; together with $9 \%$ of the $\gamma$-oxygenated substrate.

DC: $R_{f}=0.28\left(n\right.$-pentane/Et $\left.{ }_{2} \mathrm{O}, 10: 1\right)$; IR (neat): $v=2972,1720,1661,1456,1268$, 1150, 1069, 977, 744, $696 \mathrm{~cm}^{-1} ;{ }^{1} \mathrm{H}-\mathrm{NMR}\left(300 \mathrm{MHz}, \mathrm{CDCl}_{3}\right): \delta(\mathrm{ppm})=0.91(\mathrm{t}$, $J=7.4 \mathrm{~Hz}, 3 \mathrm{H}), 1.17(\mathrm{~d}, J=7.0 \mathrm{~Hz}, 3 \mathrm{H}), 1.18(\mathrm{~d}, J=7.1 \mathrm{~Hz}, 3 \mathrm{H}), 1.57-1.79(\mathrm{~m}$, $2 \mathrm{H}), 2.58(\mathrm{~m}, 1 \mathrm{H}), 5.14(\mathrm{~d}, J=12.4 \mathrm{~Hz}, 1 \mathrm{H}), 5.19(\mathrm{~d}, J=12.4 \mathrm{~Hz}, 1 \mathrm{H}), 5.35(\mathrm{~m}$, $1 \mathrm{H}), 5.97(\mathrm{dd}, J=15.8,1.6 \mathrm{~Hz}, 1 \mathrm{H}), 6.89(\mathrm{dd}, J=15.8,5.1 \mathrm{~Hz}, 1 \mathrm{H}), 7.27-7.38(\mathrm{~m}$, $5 \mathrm{H}) ;{ }^{13} \mathrm{C}-\mathrm{NMR}\left(75 \mathrm{MHz}, \mathrm{CDCl}_{3}\right): \delta(\mathrm{ppm})=9.1,18.8,19.0,26.8,34.1,66.3,73.0$, 121.0, 128.2, 128.3, 128.5, 135.7, 146.1, 165.8, 176.0; HR-MS (ESI): $\left[\mathrm{C}_{17} \mathrm{H}_{22} \mathrm{NaO}_{4}\right]^{+}$ $\left([\mathrm{M}+\mathrm{Na}]^{+}\right)$: obs.: 313.1414 ; calcd.: 313.1410 . 


\section{(E)-6-(Benzyloxy)-6-oxohex-4-en-3-yl cyclopropanecarboxylate (3q)}

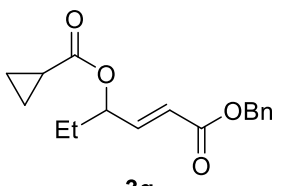

$3 \mathbf{q}$

Following the general procedure: benzyl (E)-hex-3-enoate (1a)

(204 mg, $1.00 \mathrm{mmol}, 1.0$ equiv.), (PhSe) 2 (31.2 mg, $100 \mu \mathrm{mol}$, 0.1 equiv.), ( $p$-anisyl) ${ }_{3}$ Pyr (24.3 mg, $50.0 \mu \mathrm{mol}, 0.05$ equiv.), MeCN (3 mL), cyclopropane-carboxylic acid (2 mL), $24 \mathrm{~h}$; eluting with $n$-pentane/ $/ \mathrm{t}_{2} \mathrm{O}, 10: 1$; yield: $164 \mathrm{mg}, 569 \mu \mathrm{mol}, 57 \%$, colourless liquid.

DC: $R_{f}=0.20$ (n-pentane/Et $\left.{ }_{2} \mathrm{O}, 10: 1\right)$; IR (neat): $v=2971,1719,1661,1393,1263$, 1158, 1064, 1027, 977, 912, 740, $696 \mathrm{~cm}^{-1} ;{ }^{1} \mathrm{H}-\mathrm{NMR} \quad\left(300 \mathrm{MHz}, \mathrm{CDCl}_{3}\right): \delta$ $($ ppm $)=0.78-1.08(\mathrm{~m}, 7 \mathrm{H}), 1.54-1.81(\mathrm{~m}, 3 \mathrm{H}), 5.14(\mathrm{~d}, J=12.7 \mathrm{~Hz}, 1 \mathrm{H}), 5.19(\mathrm{~d}$, $J=12.7 \mathrm{~Hz}, 1 \mathrm{H}$ ), 5.35 (tdd, $J=6.5,5.1,1.6 \mathrm{~Hz}, 1 \mathrm{H}), 5.99(\mathrm{dd}, J=15.7,1.6 \mathrm{~Hz}$, $1 \mathrm{H}), 6.88(\mathrm{dd}, J=15.7,5.1 \mathrm{~Hz}, 1 \mathrm{H}), 7.28-7.38(\mathrm{~m}, 5 \mathrm{H}) ;{ }^{13} \mathrm{C}-\mathrm{NMR}\left(75 \mathrm{MHz}, \mathrm{CDCl}_{3}\right)$ : $\delta(\mathrm{ppm})=8.5,8.6,9.2,12.8,26.8,66.3,73.2,121.1,128.2,128.3,128.5,135.7$, 146.0, 165.8, 173.9; HR-MS (ESI): $\left[\mathrm{C}_{17} \mathrm{H}_{20} \mathrm{NaO}_{4}\right]^{+}\left([\mathrm{M}+\mathrm{Na}]^{+}\right)$: obs.: 311.1255; calcd.: 311.1254.

\section{Benzyl (E)-4-((3-methylbutanoyl)oxy)hex-2-enoate (3r)}

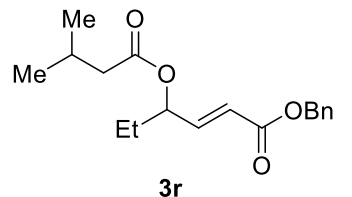

Following the general procedure: benzyl (E)-hex-3-enoate (1a) (204 mg, $1.00 \mathrm{mmol}, 1.0$ equiv.), (PhSe) 2 (31.2 mg, $100 \mu \mathrm{mol}$, 0.1 equiv. $), \quad(p \text {-anisyl })_{3}$ Pyr $(24.3 \mathrm{mg}, \quad 50.0 \mu \mathrm{mol}, 0.05$ equiv. $)$, $\operatorname{MeCN}(3 \mathrm{~mL})$, isovaleric acid $(2 \mathrm{~mL}), 24 \mathrm{~h}$; eluting with $n$-pentane/Et ${ }_{2} \mathrm{O}, 5: 1$; yield: $162 \mathrm{mg}, 529 \mu \mathrm{mol}, 53 \%$, colourless liquid.

DC: $R_{f}=0.28$ (n-pentane/Et $\left.{ }_{2} \mathrm{O}, 5: 1\right)$; IR (neat): $v=2962,1721,1661,1455,1371$, 1249, 1161, 1116, 976, 739, $696 \mathrm{~cm}^{-1}$; ${ }^{1} \mathrm{H}-\mathrm{NMR}\left(400 \mathrm{MHz}, \mathrm{CDCl}_{3}\right): \delta(\mathrm{ppm})=0.91(\mathrm{t}$, $J=7.4 \mathrm{~Hz}, 3 \mathrm{H}), 0.95(\mathrm{~d}, J=6.6 \mathrm{~Hz}, 6 \mathrm{H}), 1.62-1.76(\mathrm{~m}, 2 \mathrm{H}), 2.10(\mathrm{~m}, 1 \mathrm{H})$, 2.18-2.24 (m, 2 H), 5.16 (d, J = 12.3 Hz, 1 H), 5.17 (d, J = 12.3 Hz, 1 H), 5.36 (dddd, $J=6.7,6.0,5.2,1.6 \mathrm{~Hz}, 1 \mathrm{H}), 5.98(\mathrm{dd}, J=15.8,1.6 \mathrm{~Hz}, 1 \mathrm{H}), 6.88(\mathrm{dd}, J=15.8$, $5.2 \mathrm{~Hz}, 1 \mathrm{H}), 7.27-7.40(\mathrm{~m}, 5 \mathrm{H}) ;{ }^{13} \mathrm{C}-\mathbf{N M R}\left(101 \mathrm{MHz}, \mathrm{CDCl}_{3}\right): \delta(\mathrm{ppm})=9.3,22.4$, 22.4, 25.7, 26.9, 43.4, 66.4, 73.2, 121.3, 128.3, 128.6, 135.8, 146.0, 165.8, 172.1; HR-MS (ESI): $\left[\mathrm{C}_{18} \mathrm{H}_{24} \mathrm{NaO}_{4}\right]^{+}\left([\mathrm{M}+\mathrm{Na}]^{+}\right)$: obs.: 327.1569 ; calcd.: 327.1567 . 


\section{Benzyl (E)-4-(pivaloyloxy)hex-2-enoate (3s)}

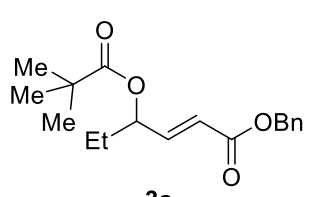

$3 s$

Following the general procedure: benzyl $(E)$-hex-3-enoate (1a) (204 mg, $1.00 \mathrm{mmol}, 1.0$ equiv.), (PhSe) 2 (31.2 mg, $100 \mu \mathrm{mol}$, 0.1 equiv.), ( $p$-anisyl) ${ }_{3} \operatorname{Pyr}(24.3 \mathrm{mg}, 50.0 \mu \mathrm{mol}, 0.05$ equiv.), $\mathrm{MeCN}$ $(3 \mathrm{~mL})$, pivalic acid $(2 \mathrm{~mL}), 48 \mathrm{~h}$; eluting with $n$-pentane/ $\mathrm{Et}_{2} \mathrm{O}, 10: 1$; yield: $78 \mathrm{mg}$, $256 \mu \mathrm{mol}, 26 \%$, yellow oil.

DC: $R_{f}=0.21\left(n\right.$-pentane/Et $\left.{ }_{2} \mathrm{O}, 10: 1\right)$; IR (neat): $v=2971,1721,1661,1456,1273$, 1146, 977, 739, $696 \mathrm{~cm}^{-1} ;{ }^{1} \mathrm{H}-\mathrm{NMR}\left(400 \mathrm{MHz}, \mathrm{CDCl}_{3}\right): \delta(\mathrm{ppm})=0.90(\mathrm{t}, J=7.4 \mathrm{~Hz}$, $3 \mathrm{H}), 1.21(\mathrm{~s}, 9 \mathrm{H}), \quad 1.56-1.80(\mathrm{~m}, 2 \mathrm{H}), 5.15(\mathrm{~d}, J=12.3 \mathrm{~Hz}, 1 \mathrm{H}), 5.19(\mathrm{~d}$, $J=12.3 \mathrm{~Hz}, 1 \mathrm{H}), 5.33(\mathrm{~m}, 1 \mathrm{H}), 5.96(\mathrm{dd}, J=15.7,1.7 \mathrm{~Hz}, 1 \mathrm{H}), 6.89(\mathrm{dd}, J=15.7$, $4.9 \mathrm{~Hz}, 1 \mathrm{H}), 7.26-7.44(\mathrm{~m}, 5 \mathrm{H}) ;{ }^{13} \mathrm{C}-\mathrm{NMR}\left(101 \mathrm{MHz}, \mathrm{CDCl}_{3}\right): \delta(\mathrm{ppm})=9.2,26.9$, 27.2, 39.0, 66.4, 73.0, 120.9, 128.3, 128.3, 128.6, 135.8, 146.3, 165.9, 177.5; HR-MS (ESI): $\left[\mathrm{C}_{18} \mathrm{H}_{24} \mathrm{NaO}_{4}\right]^{+}\left([\mathrm{M}+\mathrm{Na}]^{+}\right)$: obs.: 327.1569 ; calcd.: 327.1567 .

(E)-6-(Benzyloxy)-6-oxohex-4-en-3-yl cyclobutanecarboxylate (3t)

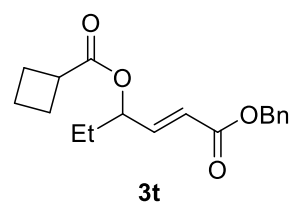

Following the general procedure: benzyl $(E)$-hex-3-enoate (1a) (204 mg, $1.00 \mathrm{mmol}, 1.0$ equiv.), (PhSe) 2 (31.2 mg, $100 \mu \mathrm{mol}$, 0.1 equiv.), ( $p$-anisyl) $)_{3}$ Pyr ( $24.3 \mathrm{mg}, 50.0 \mu \mathrm{mol}, 0.05$ equiv.), $\mathrm{MeCN}$ $(3 \mathrm{~mL})$, cyclobutanecarboxylic acid $(2 \mathrm{~mL}), 96 \mathrm{~h}$; eluting with $n$-pentane/ $\mathrm{Et}_{2} \mathrm{O}, 5: 1$; yield: $84 \mathrm{mg}, 278 \mu \mathrm{mol}, 28 \%$, colourless liquid.

DC: $R_{f}=0.25$ (n-pentane/Et $\left.{ }_{2} \mathrm{O}, 5: 1\right)$; IR (neat): $v=2970,1720,1661,1455,1376$, 1248, 1157, 1053, 977, 738, $696 \mathrm{~cm}^{-1} ;{ }^{1} \mathrm{H}-\mathrm{NMR}\left(300 \mathrm{MHz}, \mathrm{CDCl}_{3}\right): \delta(\mathrm{ppm})=0.90(\mathrm{t}$, $J=7.4 \mathrm{~Hz}, 3 \mathrm{H}), 1.57-1.79(\mathrm{~m}, 2 \mathrm{H}), 1.82-2.08(\mathrm{~m}, 2 \mathrm{H}), 2.12-2.40(\mathrm{~m}, 2 \mathrm{H}), 3.16(\mathrm{~m}$, $1 \mathrm{H}), 5.14(\mathrm{~d}, J=12.3 \mathrm{~Hz}, 1 \mathrm{H}), 5.18(\mathrm{~d}, J=12.3 \mathrm{~Hz}, 1 \mathrm{H}), 5.34(\mathrm{~m}, 1 \mathrm{H}), 5.96$ (dd, $J=15.7,1.6 \mathrm{~Hz}, 1 \mathrm{H}), 6.88(\mathrm{dd}, J=15.7,5.1 \mathrm{~Hz}, 1 \mathrm{H}), 7.25-7.46(\mathrm{~m}, 5 \mathrm{H}) ;{ }^{13} \mathrm{C}$-NMR $\left(75 \mathrm{MHz}, \mathrm{CDCl}_{3}\right): \delta(\mathrm{ppm})=9.2,18.4,25.2,25.3,26.9,38.1,66.4,73.0,121.1$, 128.3, 128.3, 128.6, 135.8, 146.1, 165.9, 174.5; HR-MS (ESI): $\left[\mathrm{C}_{18} \mathrm{H}_{22} \mathrm{NaO}_{4}\right]^{+}$ $\left([\mathrm{M}+\mathrm{Na}]^{+}\right):$obs.: 325.1413 ; calcd.: 325.1410 .

\section{Benzyl (E)-4-(2-methoxyacetoxy)hex-2-enoate (3u)}

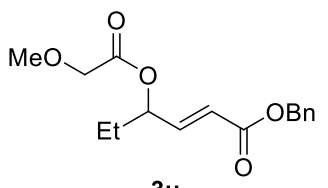

$3 \mathbf{u}$

Following the general procedure: benzyl (E)-hex-3-enoate (1a) (204 mg, $1.00 \mathrm{mmol}, 1.0$ equiv.), (PhSe) 2 (31.2 mg, $100 \mu \mathrm{mol}$, 
0.1 equiv.), ( $p$-anisyl $)_{3} \mathrm{Pyr} \quad(24.3 \mathrm{mg}, \quad 50.0 \mu \mathrm{mol}, 0.05$ equiv. $), \operatorname{MeCN} \quad(3 \mathrm{~mL})$, methoxyacetic acid $(2 \mathrm{~mL}), 24 \mathrm{~h}$; eluting with $n$-pentane/Et $\mathrm{O}_{2} \mathrm{O}, 1: 1$; yield: $179 \mathrm{mg}$, $613 \mu \mathrm{mol}, 61 \%$, colourless liquid.

DC: $R_{f}=0.31$ (n-pentane/Et ${ }_{2} \mathrm{O}, 1: 1$ ); IR (neat): $v=2937,1755,1719,1661,1455$, 1379, 1269, 1166, 1125, 978, 741, $697 \mathrm{~cm}^{-1} ;{ }^{1} \mathrm{H}-\mathrm{NMR}\left(400 \mathrm{MHz}, \mathrm{CDCl}_{3}\right): \delta$ $(\mathrm{ppm})=0.91(\mathrm{t}, \quad J=7.4 \mathrm{~Hz}, 3 \mathrm{H}), 1.66-1.79(\mathrm{~m}, 2 \mathrm{H}), 3.43(\mathrm{~s}, 3 \mathrm{H}), 4.01$ (d, $J=15.8 \mathrm{~Hz}, 1 \mathrm{H}), 4.08(\mathrm{~d}, J=15.8 \mathrm{~Hz}, 1 \mathrm{H}), 5.13(\mathrm{~d}, J=12.5 \mathrm{~Hz}, 1 \mathrm{H}), 5.18(\mathrm{~d}$, $J=12.5 \mathrm{~Hz}, 1 \mathrm{H}), 5.44(\mathrm{~m}, 1 \mathrm{H}), 5.99(\mathrm{dd}, J=15.8,1.5 \mathrm{~Hz}, 1 \mathrm{H}), 6.87(\mathrm{dd}, J=15.8$, $5.4 \mathrm{~Hz}, 1 \mathrm{H}), 7.28-7.37(\mathrm{~m}, 5 \mathrm{H}) ;{ }^{13} \mathrm{C}-N M R\left(101 \mathrm{MHz}, \mathrm{CDCl}_{3}\right): \delta(\mathrm{ppm})=9.2,26.8$, 59.4, 66.5, 69.7, 74.0, 121.8, 128.3, 128.5, 135.7, 145.1, 165.6, 169.4; HR-MS (ESI): $\left[\mathrm{C}_{16} \mathrm{H}_{20} \mathrm{NaO}_{5}\right]^{+}\left([\mathrm{M}+\mathrm{Na}]^{+}\right)$: obs.: 315.1204; calcd.: 315.1203 .

\section{Benzyl (E)-4-(2,2,2-trifluoroacetoxy)hex-2-enoate (3v)}

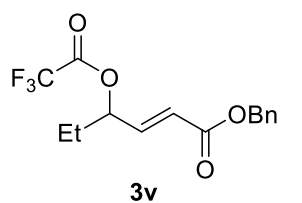

Following the general procedure: benzyl $(E)$-hex-3-enoate (1a) (204 mg, $1.00 \mathrm{mmol}, 1.0$ equiv.), (PhSe) 2 (31.2 mg, $100 \mu \mathrm{mol}$, 0.1 equiv.), ( $p$-anisyl) $)_{3} P y r(24.3 \mathrm{mg}, 50.0 \mu \mathrm{mol}, 0.05$ equiv.), MeCN $(3 \mathrm{~mL})$, trifluoroacetic acid $(2 \mathrm{~mL}), 24 \mathrm{~h}$; eluting with $n$-pentane/ $\mathrm{Et}_{2} \mathrm{O}, 3: 1$; yield: $218 \mathrm{mg}, 689$ umol, $69 \%$, colourless liquid.

DC: $R_{f}=0.26$ (n-pentane/Et ${ }_{2} \mathrm{O}, 3: 1$ ); IR (neat): $v=2976,1784,1722,1661,1309$, 1272, 1220, 1148, 976, 730, $696 \mathrm{~cm}^{-1}$; ${ }^{1} \mathrm{H}-\mathrm{NMR}\left(300 \mathrm{MHz}, \mathrm{CDCl}_{3}\right): \delta(\mathrm{ppm})=0.96(\mathrm{t}$, $J=7.4 \mathrm{~Hz}, 3 \mathrm{H}), 1.75-1.91(\mathrm{~m}, 2 \mathrm{H}), 5.18(\mathrm{~d}, J=12.4 \mathrm{~Hz}, 1 \mathrm{H}), 5.19(\mathrm{~d}, J=12.4 \mathrm{~Hz}$, $1 \mathrm{H}), 5.49(\mathrm{qd}, J=6.3,1.5 \mathrm{~Hz}, 1 \mathrm{H}), 6.05(\mathrm{dd}, J=15.8,1.5 \mathrm{~Hz}, 1 \mathrm{H}), 6.86$ (dd, $J=15.8,5.6 \mathrm{~Hz}, 1 \mathrm{H}), 7.26-7.44(\mathrm{~m}, 5 \mathrm{H}) ;{ }^{13} \mathrm{C}-\mathbf{N M R}\left(75 \mathrm{MHz} \mathrm{CDCl}_{3}\right): \delta(\mathrm{ppm})=8.9$, 26.7, 66.7, 77.9, 114.3 (q, $J=285.9 \mathrm{~Hz}$ ), 123.1, 128.4, 128.4, 128.6, 135.5, 142.5, $156.6(q, J=42.8 \mathrm{~Hz}), 165.2 ;{ }^{19} \mathrm{~F}-\mathrm{NMR}\left(283 \mathrm{MHz}, \mathrm{CDCl}_{3}\right): \delta(\mathrm{ppm})=-75.0 ;$ HR-MS (ESI): $\left[\mathrm{C}_{15} \mathrm{H}_{15} \mathrm{~F}_{3} \mathrm{NaO}_{4}\right]^{+}\left([\mathrm{M}+\mathrm{Na}]^{+}\right)$: obs.: 339.0822; calcd.: 339.0815 .

\section{Benzyl (E)-4-(2-bromoacetoxy)hex-2-enoate (3w)}

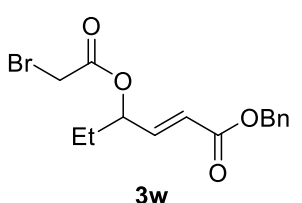

Following the general procedure: benzyl (E)-hex-3-enoate (1a) (204 mg, $1.00 \mathrm{mmol}, 1.0$ equiv.), (PhSe) 2 (31.2 mg, $100 \mu \mathrm{mol}$, 0.1 equiv.), ( $p$-anisyl) ${ }_{3}$ Pyr (24.3 mg, $50.0 \mu \mathrm{mol}, 0.05$ equiv.), $\mathrm{MeCN}$ $(3 \mathrm{~mL})$, bromoacetic acid $(2 \mathrm{~mL}), 24 \mathrm{~h}$; eluting with $n$-pentane/ $\mathrm{Et}_{2} \mathrm{O}, 3: 1$; yield: $180 \mathrm{mg}, 528 \mu \mathrm{mol}, 53 \%$, colourless liquid. 
DC: $R_{f}=0.31$ (n-pentane/Et $\left.{ }_{2} \mathrm{O}, 3: 1\right)$; IR (neat): $v=2971,1718,1661,1267,1163$, 1106, 975, 739, $696 \mathrm{~cm}^{-1} ;{ }^{1} \mathrm{H}-\mathrm{NMR}\left(300 \mathrm{MHz}, \mathrm{CDCl}_{3}\right): \delta(\mathrm{ppm})=0.94$ (t, $J=7.4 \mathrm{~Hz}$, $3 \mathrm{H}$ ), 1.60-1.85 (m, $2 \mathrm{H}$ ), 3.84 (s, $2 \mathrm{H}$ ), 5.17 (s, $2 \mathrm{H}), 5.38(\mathrm{~m}, 1 \mathrm{H}), 6.04(\mathrm{dd}, J=15.8$, $1.5 \mathrm{~Hz}, 1 \mathrm{H}), 6.87$ (dd, $J=15.8,5.3 \mathrm{~Hz}, 1 \mathrm{H}), 7.27-7.41(\mathrm{~m}, 5 \mathrm{H}) ;{ }^{13} \mathrm{C}-\mathrm{NMR}(75 \mathrm{MHz}$, $\left.\mathrm{CDCl}_{3}\right): \delta(\mathrm{ppm})=9.1,25.5,26.7,66.4,75.3,121.9,128.3,128.5,135.6,144.5$, 165.5, 166.3; HR-MS (ESI): $\left[\mathrm{C}_{15} \mathrm{H}_{17} \mathrm{BrNaO}_{4}\right]^{+}\left([\mathrm{M}+\mathrm{Na}]^{+}\right)$: obs.: 363.0205 ; calcd.: 363.0202 .

\section{Benzyl (E)-4-(2-bromoacetoxy)hex-2-enoate (3x)}

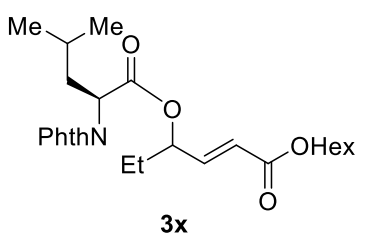

Following the general procedure: hexyl $(E)$-hex-3-enoate (1c) (200 mg, $1.01 \mathrm{mmol}, 1.0$ equiv.), (PhSe) 2 (31.2 mg, $100 \mu \mathrm{mol}$, 0.1 equiv.), ( $p$-anisyl) $)_{3}$ Pyr (24.3 mg, $50.0 \mu \mathrm{mol}, 0.05$ equiv.), MeCN $(5 \mathrm{~mL}), \quad L-N$-phthalyl leucine $(1.31 \mathrm{~g}, 5.00 \mathrm{mmol}$, 5.0 equiv.), molecular sieves (powdered, $4 \AA, 30 \mathrm{mg}$ ), $\mathrm{O}_{2}$ atmosphere, $16 \mathrm{~h}$; eluting with $n$-pentane/Et ${ }_{2} \mathrm{O}, 3: 1$; yield: $315 \mathrm{mg}, 688 \mu \mathrm{mol}, 68 \%$, $d r 1: 1$ yellow oil.

DC: $R_{f}=0.32\left(n\right.$-pentane/Et $\left.{ }_{2} \mathrm{O}, 3: 1\right) ;$ IR (neat): $v=2957,1712,1384,1247,1173$, 1056, 978, 718, $529 \mathrm{~cm}^{-1} ;{ }^{1} \mathrm{H}-\mathrm{NMR}\left(500 \mathrm{MHz}, \mathrm{CDCl}_{3}\right): \delta(\mathrm{ppm})=0.72-1.03(\mathrm{~m}, 12 \mathrm{H})$, 1.15-1.40 (m, $6 \mathrm{H}), 1.41-1.80(\mathrm{~m}, 5 \mathrm{H}), 1.94$ (dddd, $J=14.4,10.3,8.4,4.4 \mathrm{~Hz}, 1 \mathrm{H}$ ), $2.35(\mathrm{~m}, 1 \mathrm{H}), 4.00-4.18(\mathrm{~m}, 2 \mathrm{H}), 4.97$ (ddd, $J=11.4,7.1,4.4 \mathrm{~Hz}, 1 \mathrm{H}), 5.37(\mathrm{~m}$, $1 \mathrm{H}$ ), 5.87 (ddd, $J=15.8,10.3,1.6 \mathrm{~Hz}, 1 \mathrm{H}), 6.75$ (ddd, $J=15.8,5.5,3.1 \mathrm{~Hz}, 1 \mathrm{H}$ ), 7.64-7.77 (m, $2 \mathrm{H}), 7.80-7.91(\mathrm{~m}, 2 \mathrm{H}) ;{ }^{13} \mathrm{C}-\mathrm{NMR}\left(126 \mathrm{MHz}, \mathrm{CDCl}_{3}\right): \delta(\mathrm{ppm})=9.0$, 9.1, 14.0, 14.0, 21.0, 21.0, 22.5, 23.2, 23.2, 25.0, 25.1, 25.6, 26.8, 26.9, 28.5, 31.4, $31.4,37.2,37.2,50.7,50.9,64.8,64.8,75.1,75.2,122.2,122.2,123.5,123.5,123.6$, 131.7, 131.7, 134.2, 134.2, 134.3, 144.1, 144.1, 166.0, 166.0, 167.7, 167.7, 169.0, 169.0; HR-MS (ESI): $\left[\mathrm{C}_{26} \mathrm{H}_{36} \mathrm{NO}_{6}\right]^{+}\left([\mathrm{M}+\mathrm{H}]^{+}\right)$: obs.: 458.2540; calcd.: 458.2537. 


\section{Selenofunctionalization and elimination experiments}

In order to obtain preliminary insights into the mechanism of the title procedure we performed a series of control experiments (Table S4). From crude ${ }^{1} \mathrm{H}$ NMR spectra we identified selenofunctionalization intermediates 7 as a constituent of the reaction mixture. We therefore wanted to determine whether or not this compound is a component of the catalytic cycle. Thus, we synthesized selenide 7a through the reaction of ester 1a with $\mathrm{PhSeBr}$ in a mixture of $\mathrm{AcOH}$ and acetic anhydride (10:1, $\mathrm{v} / \mathrm{v}$ ) using KOAc as a base (see below). Subsequently, selenide 7a was exposed to the standard conditions, which furnished allylic ester 3a only in 18\% (Entry 1). Conversion of selenide $7 \mathrm{a}$ in the absence of photocatalyst 4 but under otherwise unaltered conditions led again to product formation in low yields (16\%, Entry 2). A different outcome was observed when the reaction was performed in the absence of $(\mathrm{PhSe})_{2}$ (Entry 3). In the course of $40 \mathrm{~h}$ we recorded the formation of product 3a in $62 \%$. Finally, we wanted to find out whether selenide $7 \mathrm{a}$ can be used as a precatalyst. Consequently, $20 \mathrm{~mol} \%$ of selenide 7 a were combined with alkene 1a in the presence of $5 \mathrm{~mol} \%$ of photocatalyst 4 in acetonitrile under irradiation (465 nm) and an atmosphere of air. These conditions led to a combined yield of $70 \%$ of ester 3a, thus, indicating that the active catalyst can be directly generated from selenoesterification intermediate 7a (Entry 4). Furthermore, we treated compound 7a with 1 equiv of $\mathrm{PhSeBr}$ in order to simulate the potential attack of selenide $7 \mathrm{a}$ by another selenium electrophile. However, under these conditions no product formation was observed (Entry 5). From these findings we conclude, that the elimination step does not proceed via a diselenonium intermediate, but rather via direct extrusion of the PhSe group. 
Table S4: Product formation from the selenofunctionalization product

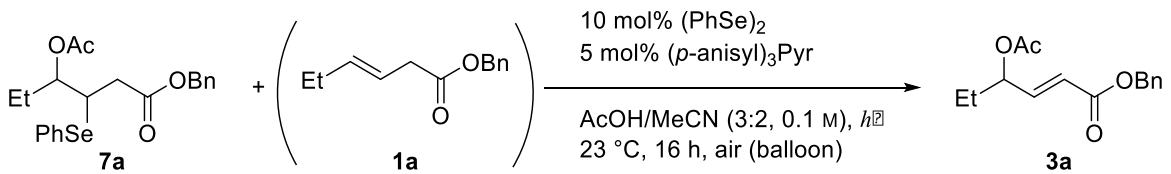

\begin{tabular}{cccccc}
\hline entry & $(\mathrm{PhSe})_{2}[\mathrm{~mol} \%]$ & $(p \text {-anisyl })_{3}$ Pyr [mol\%] & 7a [mol\%] & 1a [mol\%] & yield $(\%)$ \\
\hline 1 & 10 & 5 & 100 & - & 18 \\
2 & 10 & - & 100 & - & 16 \\
3 & - & 5 & 100 & - & 62 \\
4 & - & 5 & 20 & 100 & 70 \\
$5^{[a]}$ & - & - & 100 & - & 0 \\
\hline
\end{tabular}

[a] $\mathrm{PhSeBr}$ (1 equiv) was used.

\section{Benzyl 4-acetoxy-3-(phenylselanyl)hexanoate (7a)}

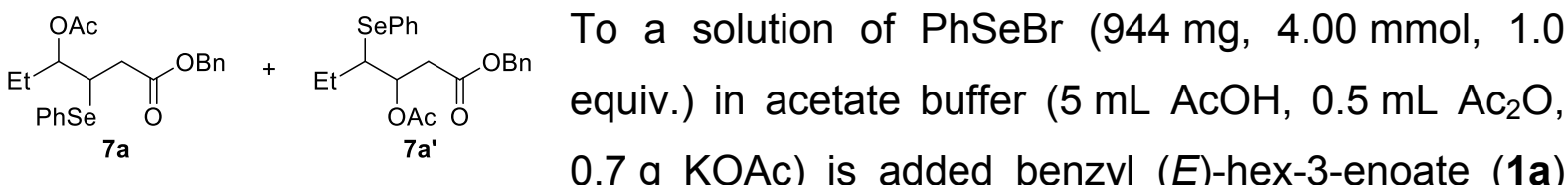

( $817 \mathrm{mg}, 4.00 \mathrm{mmol}, 1.0$ equiv.) and the resulting mixture is stirred at ambient temperature for $1 \mathrm{~h}$. DCM $(50 \mathrm{~mL})$ is added and the solution is washed with sat. aqueous $\mathrm{NaHCO}_{3}$-solution $(2 \times 50 \mathrm{~mL}$ ). The aqueous layer is back extracted with DCM $(50 \mathrm{~mL})$. The combined organic layers are dried over $\mathrm{Na}_{2} \mathrm{SO}_{4}$, the solid is filtered off and the solvents are removed under reduced pressure. The residue is purified by column chromatography on silica gel using EtOAc/n-pentane (1:10) as the eluent. The title compound is obtained as a colourless oil $(1.38 \mathrm{~g}, 3.29 \mathrm{mmol}, 82 \%)$ together with $20 \%$ of the regioisomeric selenofunctionalization product $7 a^{\prime}$.

DC: $R_{f}=0.19$ (n-pentane/EtOAc, 10:1); IR (neat): $v=2969,1732,1476,1455,1437$, 1371, 1227, 1186, 737, $692 \mathrm{~cm}^{-1}$; ${ }^{1} \mathrm{H}-\mathrm{NMR}\left(400 \mathrm{MHz}, \mathrm{CDCl}_{3}\right): \delta(\mathrm{ppm})=7 \mathrm{a}: 0.82$ (t, $J=7.4 \mathrm{~Hz}, 3 \mathrm{H}), 1.64-1.78(\mathrm{~m}, 2 \mathrm{H}), 1.90(\mathrm{~s}, 3 \mathrm{H}), 2.65(\mathrm{dd}, J=16.2,9.2 \mathrm{~Hz}, 1 \mathrm{H})$, 2.82 (dd, $J=16.2,5.2 \mathrm{~Hz}, 1 \mathrm{H}$ ), 3.66 (ddd, $J=9.2,6.0,5.2 \mathrm{~Hz}, 1 \mathrm{H}$ ), 4.99 (ddd, $J=7.7,6.0,4.8 \mathrm{~Hz}, 1 \mathrm{H}), 5.08-5.19(\mathrm{~m}, 2 \mathrm{H}), 7.19-7.37(\mathrm{~m}, 8 \mathrm{H}), 7.57$ (dd, J = 7.9, $1.6 \mathrm{~Hz}, 2 \mathrm{H}$ ); 7a': 1.12 (t, J=7.3 Hz, $3 \mathrm{H}$ ), 1.41-1.57 (m, $2 \mathrm{H}), 1.67$ (s, $3 \mathrm{H}$ ), 2.78 (dd, $J=15.9,4.5 \mathrm{~Hz}, 1 \mathrm{H}), 2.89$ (dd, $J=15.9,8.8 \mathrm{~Hz}, 1 \mathrm{H}$ ), 3.32 (ddd, $J=9.7,5.4,4.5 \mathrm{~Hz}$, $1 \mathrm{H}), 5.03-5.12(\mathrm{~m}, 2 \mathrm{H}), 5.39$ (ddd, $J=9.0,5.4,3.9 \mathrm{~Hz}, 1 \mathrm{H}), 7.19-7.38(\mathrm{~m}, 8 \mathrm{H})$, 7.52-7.56 (m, $2 \mathrm{H}) ;{ }^{13} \mathrm{C}-\mathrm{NMR}\left(101 \mathrm{MHz}, \mathrm{CDCl}_{3}\right): \delta(\mathrm{ppm})=9.6,12.8,20.4,20.7,24.6$, 
25.2, 37.0, 37.1, 43.1, 51.6, 66.5, 66.7, 72.9, 76.9, 127.6, 128.1, 128.2, 128.3, 128.4, $128.5,129.0,129.1,134.8,135.3,135.7,170.0,170.2,170.3,171.2 ;{ }^{77}$ Se-NMR $\left(76 \mathrm{MHz}, \mathrm{CDCl}_{3}\right): \delta(\mathrm{ppm})=7 \mathrm{a}: 370.6 ; 7 a^{\prime}: 317.4$; HR-MS (ESI): $\left[\mathrm{C}_{21} \mathrm{H}_{24} \mathrm{NaO}_{4} \mathrm{Se}\right]^{+}$ $\left([\mathrm{M}+\mathrm{Na}]^{+}\right):$obs.: 443.0737 ; calcd.: 443.0733 .

\section{Elimination experiments}

To a solution of compound 7a and the alkene 1a in $\mathrm{MeCN} / \mathrm{AcOH}(5 \mathrm{~mL}, 3: 2)$ are added $(\mathrm{PhSe})_{2} \quad(15.6 \mathrm{mg}, \quad 50.0 \mu \mathrm{mol}, 0.1 \text { equiv.) and ( } p \text {-anisyl) })_{3} \mathrm{Pyr} \quad(12.1 \mathrm{mg}$, $25.0 \mu \mathrm{mol}, 0.05$ equiv.) and the resulting mixture is stirred under irradiation at $\lambda=465 \mathrm{~nm}$ for $16 \mathrm{~h}$ at ambient temperature under air. The solvents are removed under reduced pressure and the residue is purified by column chromatography on silica gel using $n$-pentane/ $\mathrm{Et}_{2} \mathrm{O}(5: 1)$ as the eluent. 


\section{Interrupted irradiation experiment}

A solution of hexyl (E)-hex-3-enoate (1d) (99.2 mg, $500 \mu \mathrm{mol}, 1.0$ equiv.), (PhSe) 2 (15.6 mg, $50.0 \mu \mathrm{mol}, 0.1$ equiv.), ( $p$-anisyl) ${ }_{3} P y r(12.1 \mathrm{mg}, 25.0 \mu \mathrm{mol}, 0.05$ equiv.), a few droplets 1,1,2,2-tetrachloroethane as internal standard and acetic acid (1 mL) in $\mathrm{CD}_{3} \mathrm{CN}(4 \mathrm{~mL})$ is stirred vigorously under ambient air. The mixture is irradiated at $\lambda=465 \mathrm{~nm}$ and the yield of the acetoxylation product is determined every $30 \mathrm{~min}$ by ${ }^{1} \mathrm{H}-\mathrm{NMR}$. After irradiating for 120 min the reaction flask is wrapped in aluminum foil and irradiation is stopped. Determination of the ${ }^{1} \mathrm{H}-\mathrm{NMR}$ yield is continued. After $120 \mathrm{~min}$, irradiation of the flask is resumed for another $120 \mathrm{~min}$ followed by light exclusion for the final $120 \mathrm{~min}$.

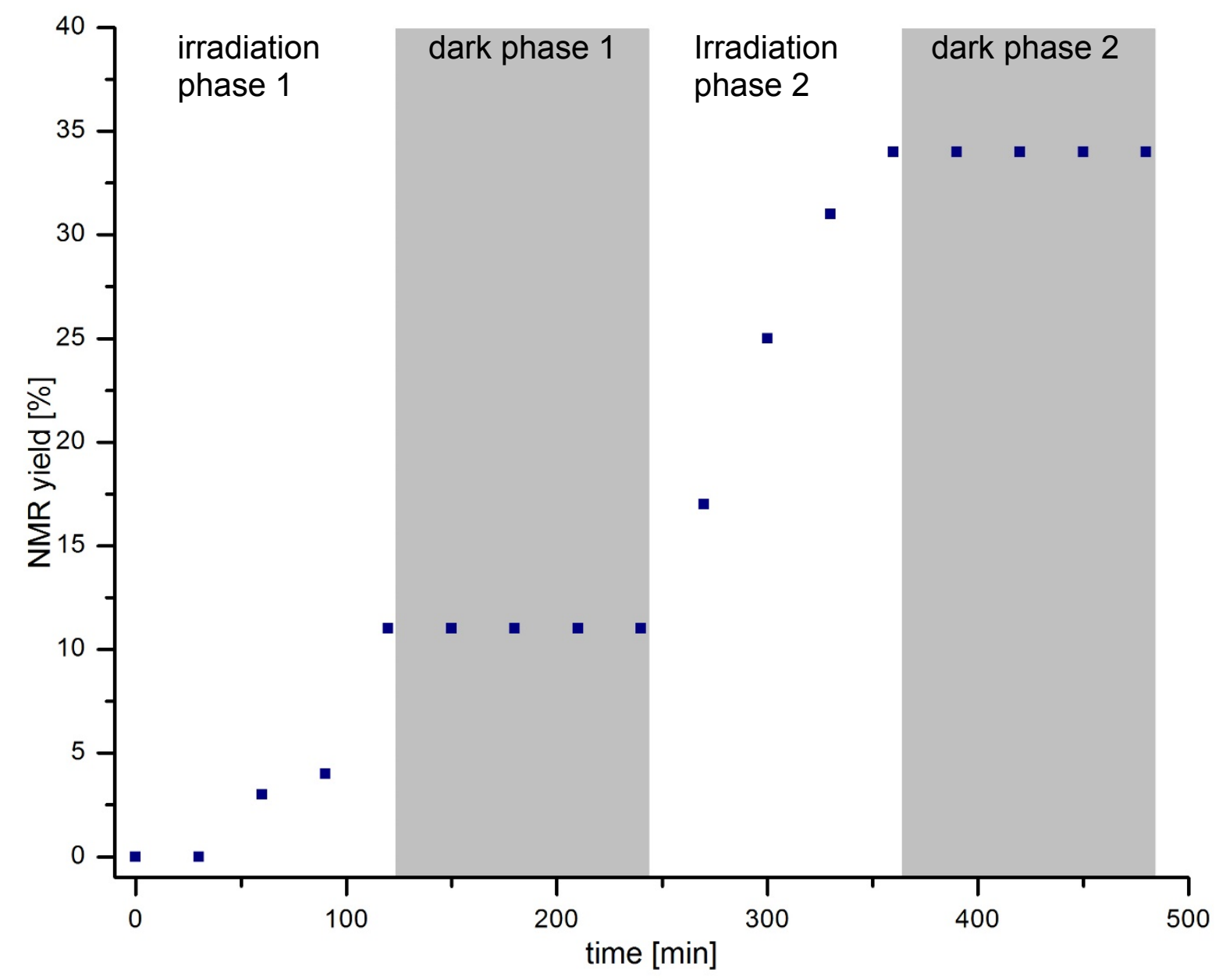

Figure S4: Interrupted irradiation experiment. 


\section{Fluorescence quenching experiments ${ }^{1}$}

A degassed solution of ( $p$-anisyl) $)_{3} \mathrm{Pyr}$ in $\mathrm{MeCN}(100 \mu \mathrm{M})$ containing the appropriate amount of quencher is excited at $\lambda=465 \mathrm{~nm}$ and the emission intensities are recorded at $\lambda=539 \mathrm{~nm}$. $(\mathrm{PhSe})_{2}$ and hexyl $(E)$-hex-3-enoate (1d) are used as fluorescence quenchers. Measurements are conducted in $10 \mathrm{~mm}$ quartz cuvettes under a nitrogen atmosphere. Plots are created according to the Stern-Vollmer equation

$$
\frac{\mathrm{I}_{0}}{\mathrm{I}}=1+\mathrm{k}_{\mathrm{q}} \cdot \tau_{0}[\mathrm{Q}]
$$

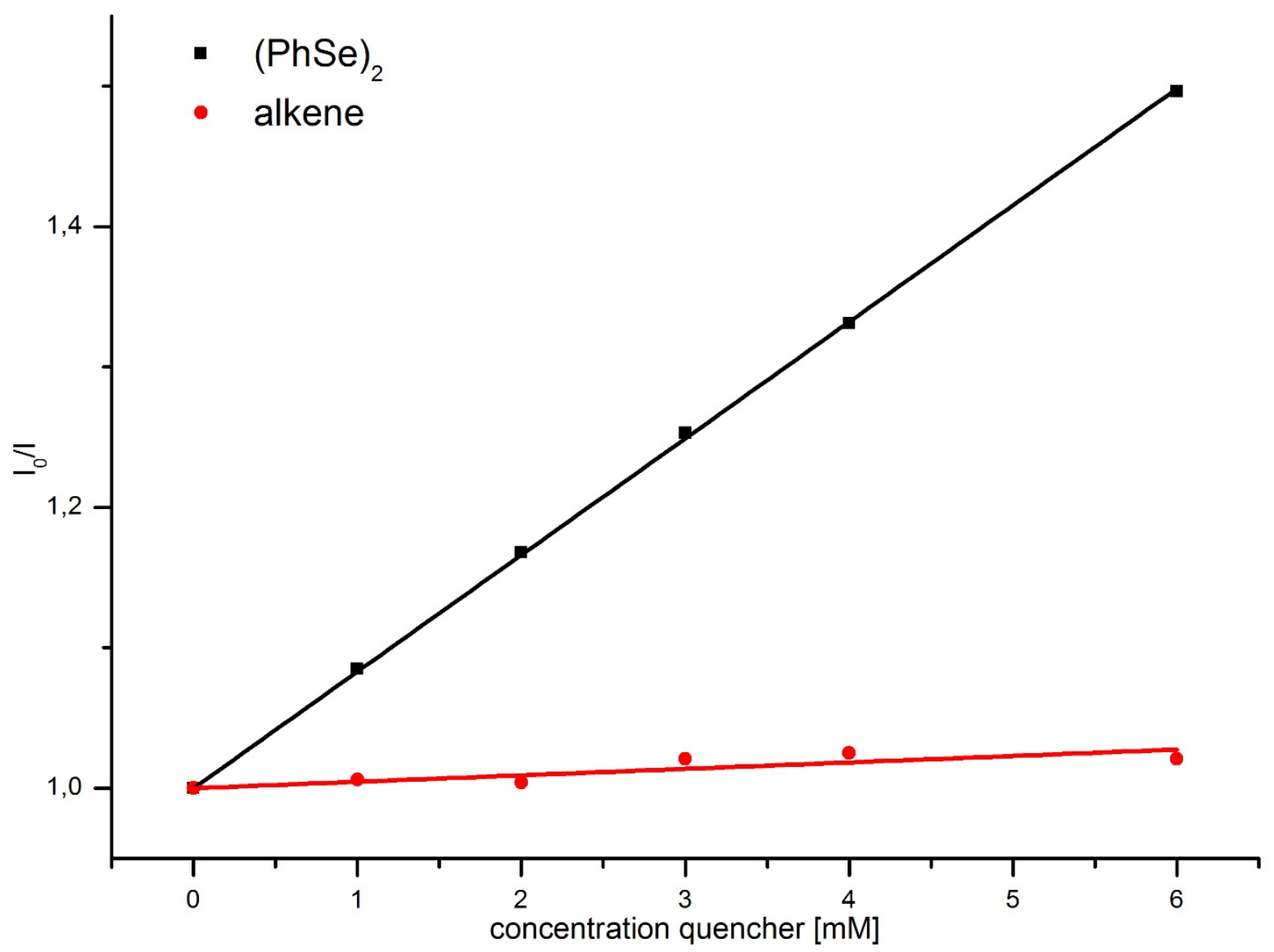

Figure S5: Plot of fluorescence quenching experiments.

\footnotetext{
${ }^{1}$ D. Hager, D. W. C. MacMillan, J. Am. Chem. Soc. 2014, 136, 16986-16989.
} 


\section{H-/13C-/IR-spectra}

${ }^{1}$ H-NMR (300 MHz), ${ }^{13}$ C-NMR (126 MHz): DMSO-D 6
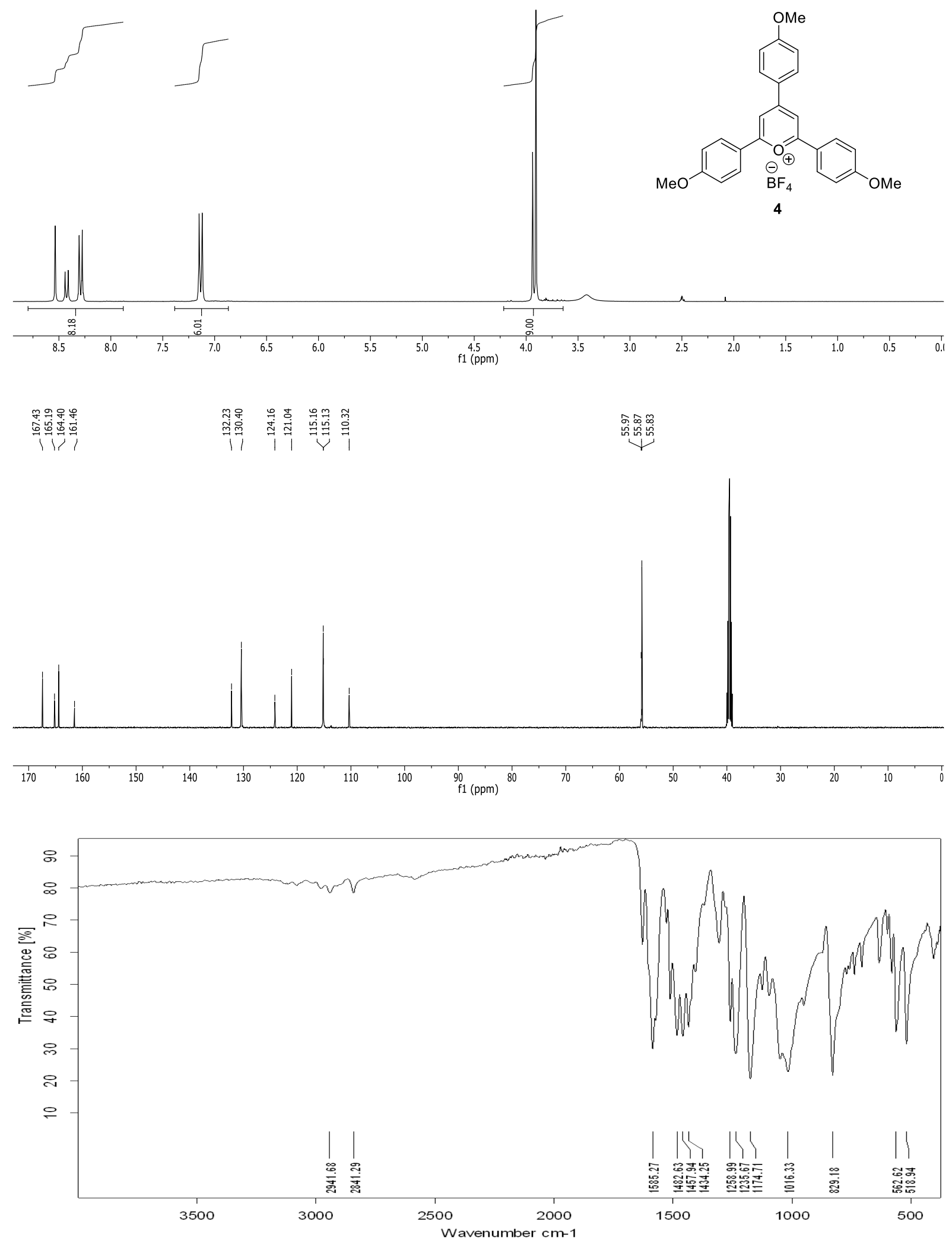
${ }^{1} \mathrm{H}-\mathrm{NMR}(300 \mathrm{MHz}),{ }^{13} \mathrm{C}-\mathrm{NMR}(75 \mathrm{MHz}): \mathrm{CDCl}_{3}$
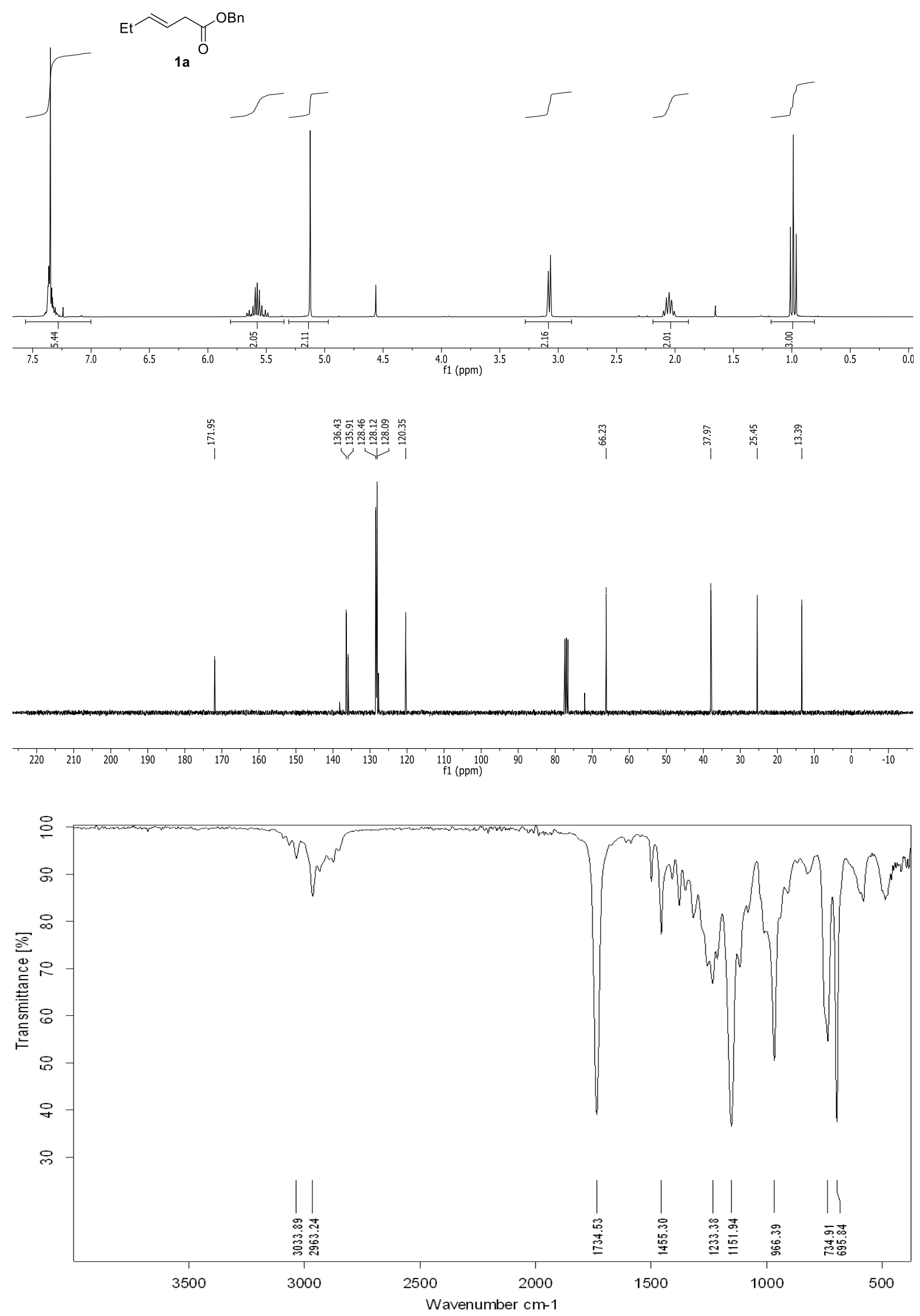
${ }^{1} \mathrm{H}-\mathrm{NMR}(300 \mathrm{MHz}),{ }^{13} \mathrm{C}$-NMR (126 MHz): $\mathrm{CDCl}_{3}$
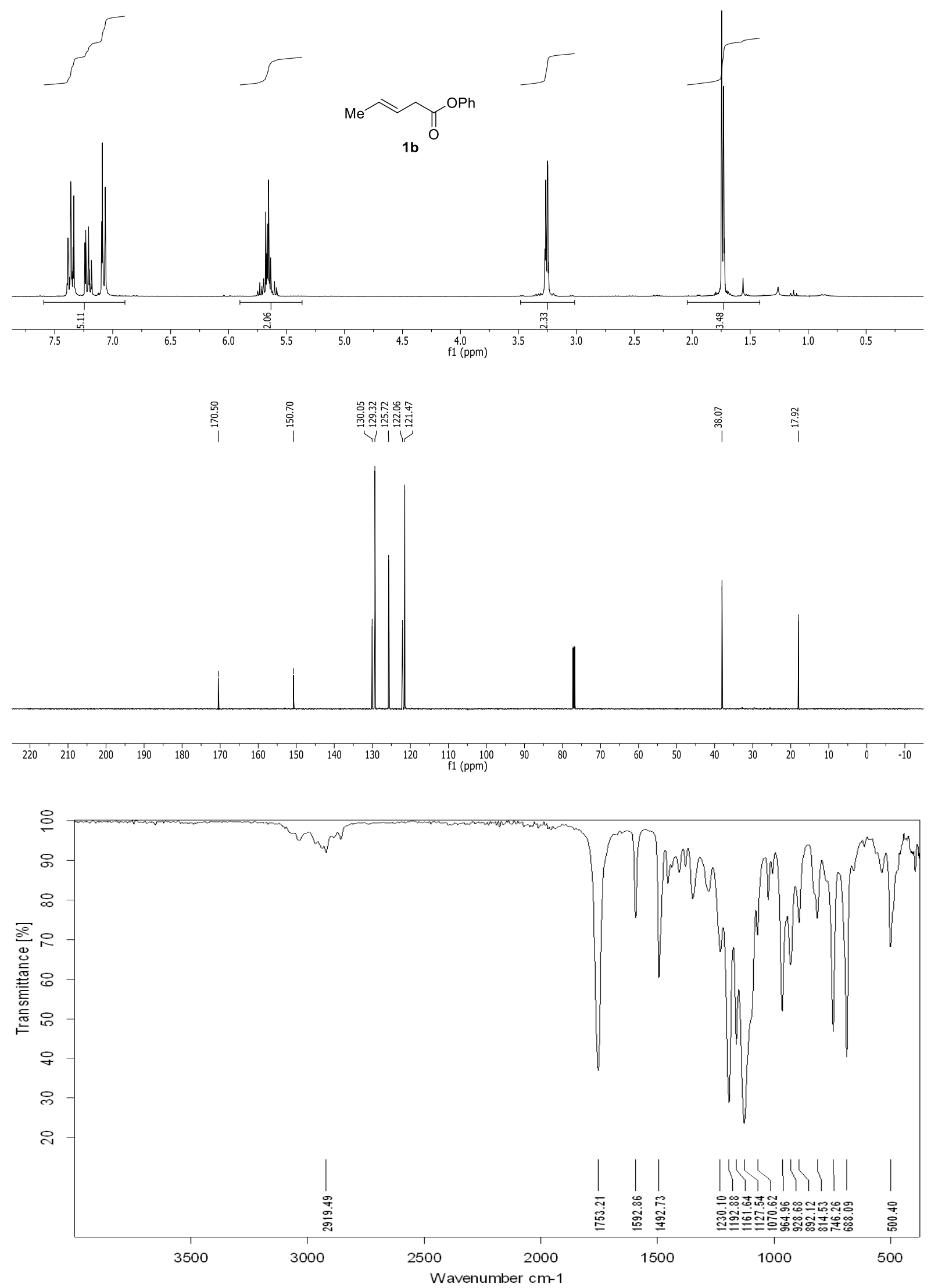
${ }^{1} \mathrm{H}-\mathrm{NMR}(300 \mathrm{MHz}),{ }^{13} \mathrm{C}$-NMR (126 MHz): $\mathrm{CDCl}_{3}$
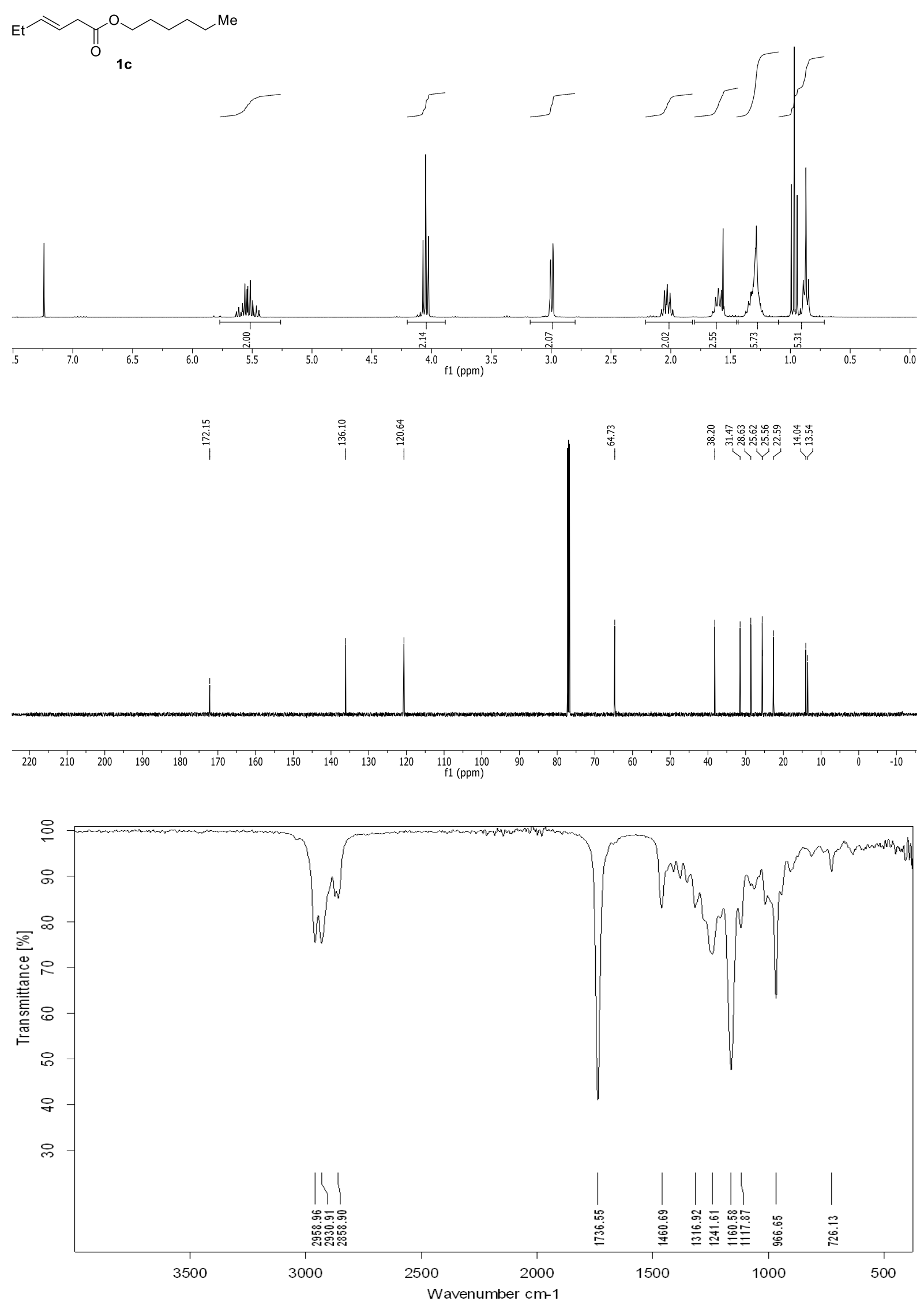
${ }^{1} \mathrm{H}-\mathrm{NMR}(400 \mathrm{MHz}),{ }^{13} \mathrm{C}$-NMR (101 MHz): $\mathrm{CDCl}_{3}$
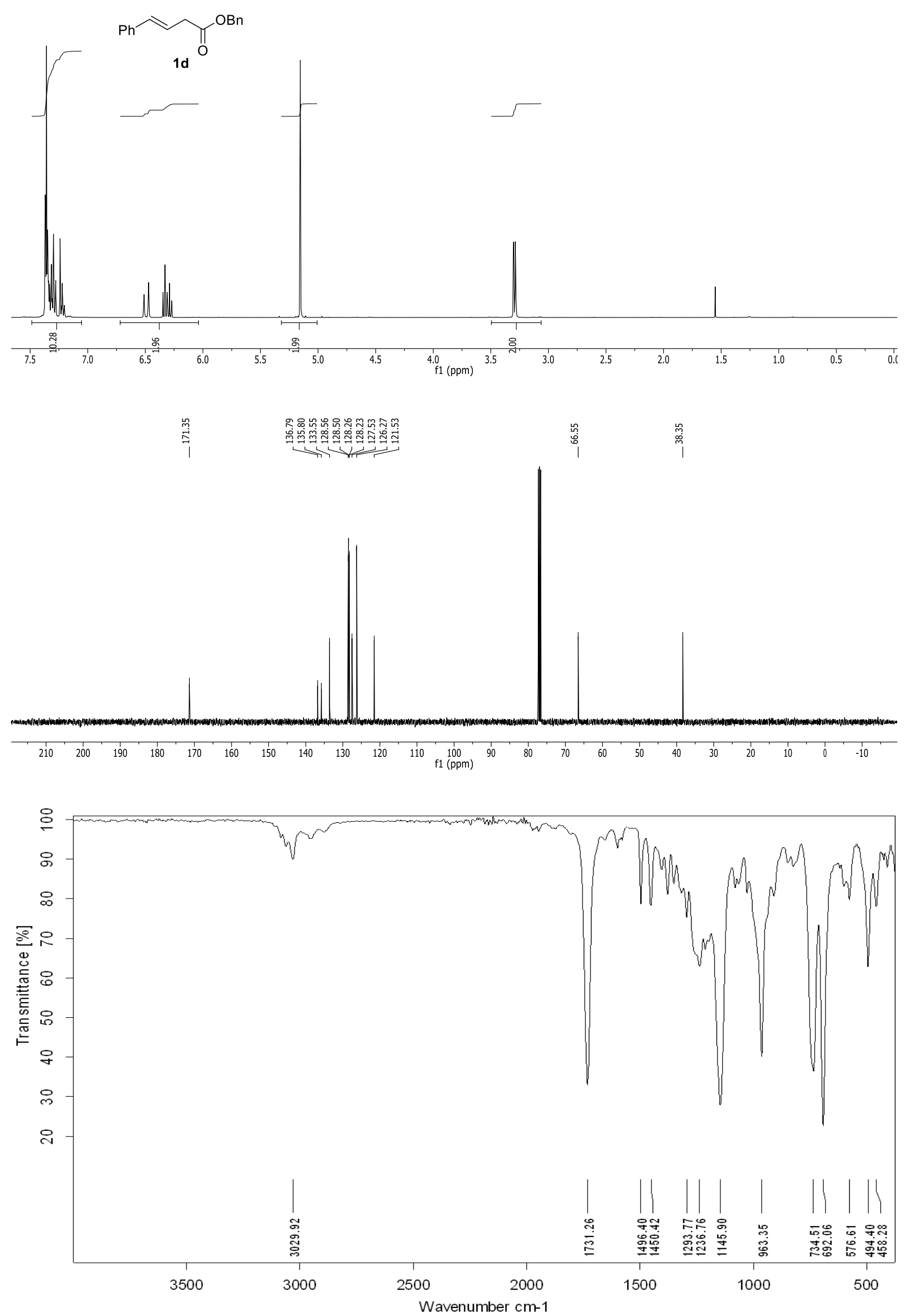
${ }^{1} \mathrm{H}-\mathrm{NMR}(300 \mathrm{MHz}),{ }^{13} \mathrm{C}$-NMR (126 MHz): $\mathrm{CDCl}_{3}$
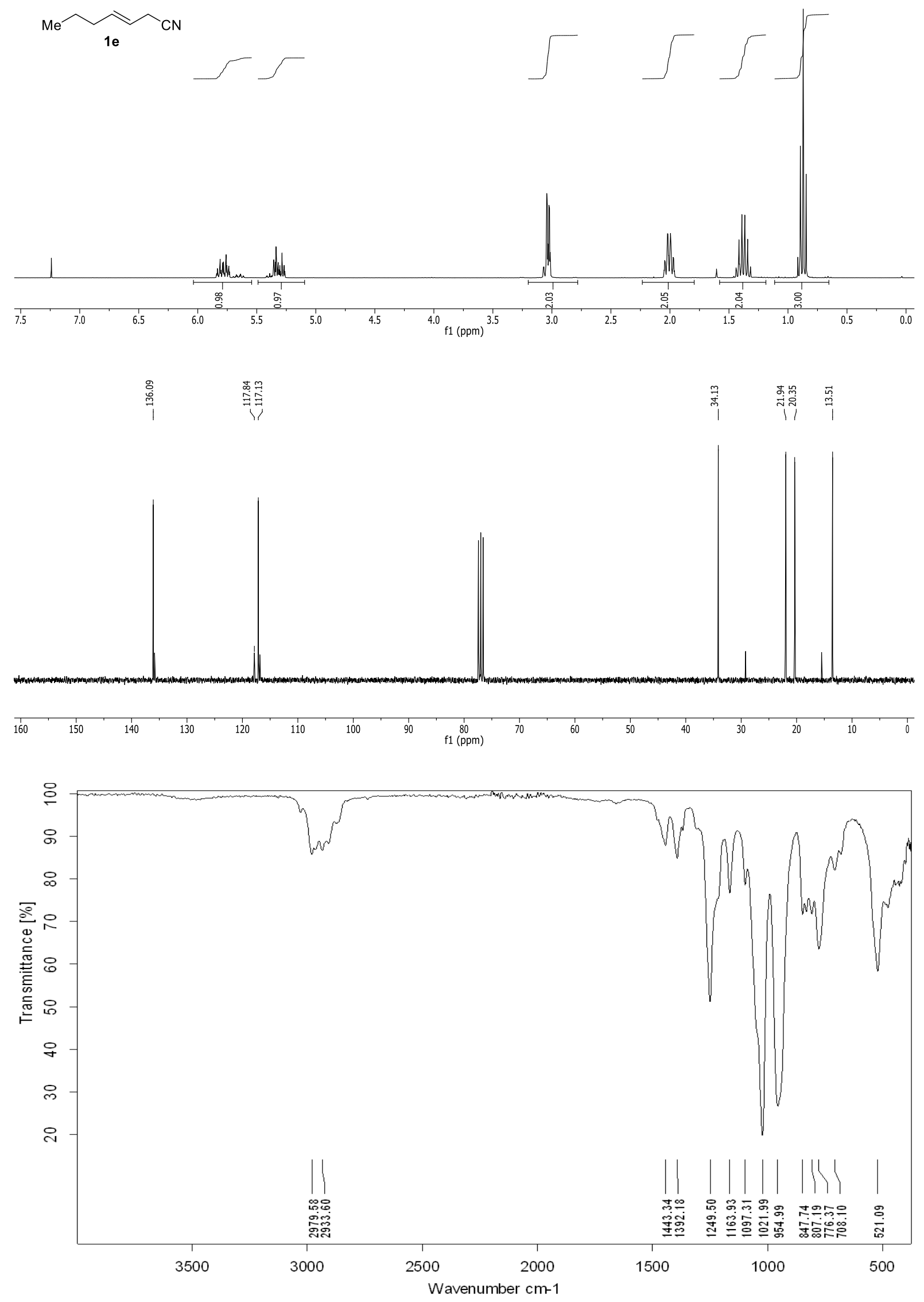
${ }^{1} \mathrm{H}-\mathrm{NMR}(500 \mathrm{MHz}),{ }^{13} \mathrm{C}$-NMR (126 MHz): $\mathrm{CDCl}_{3}$

$\mathrm{Me} \overbrace{\text { 1f }}^{\mathrm{PO}(\mathrm{OEt})_{2}}$

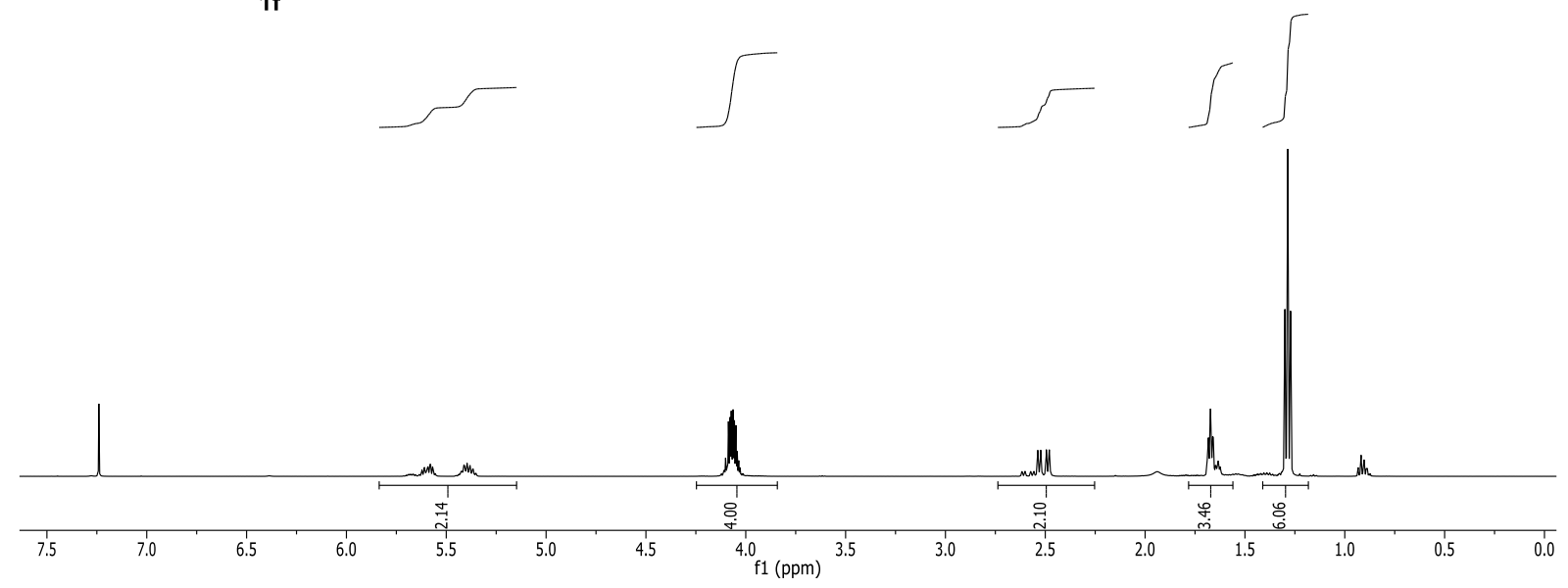

商䓞

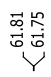

1)

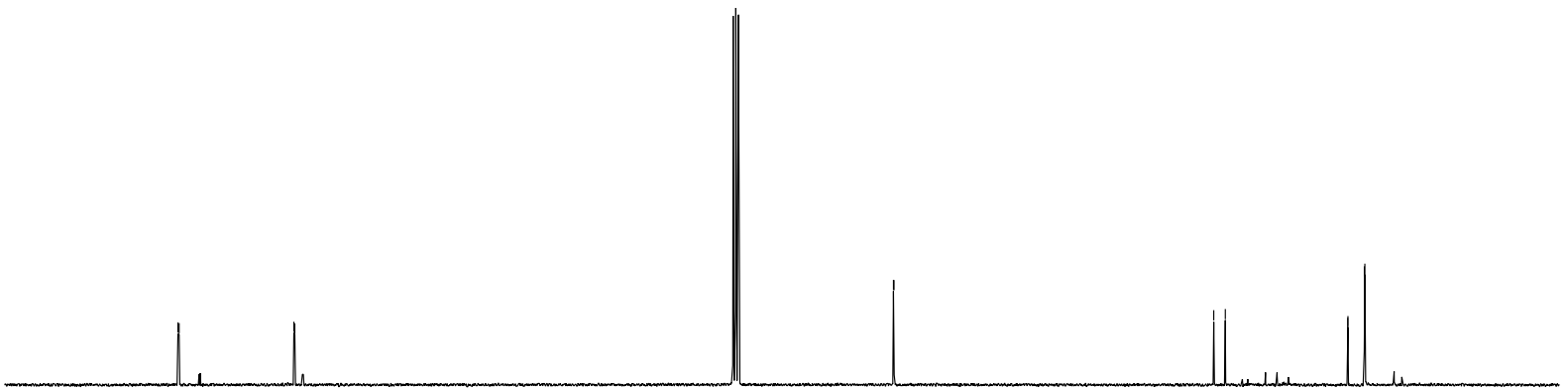

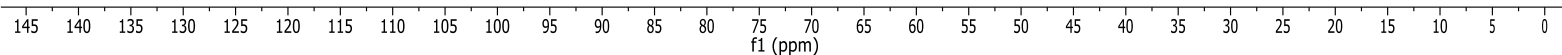

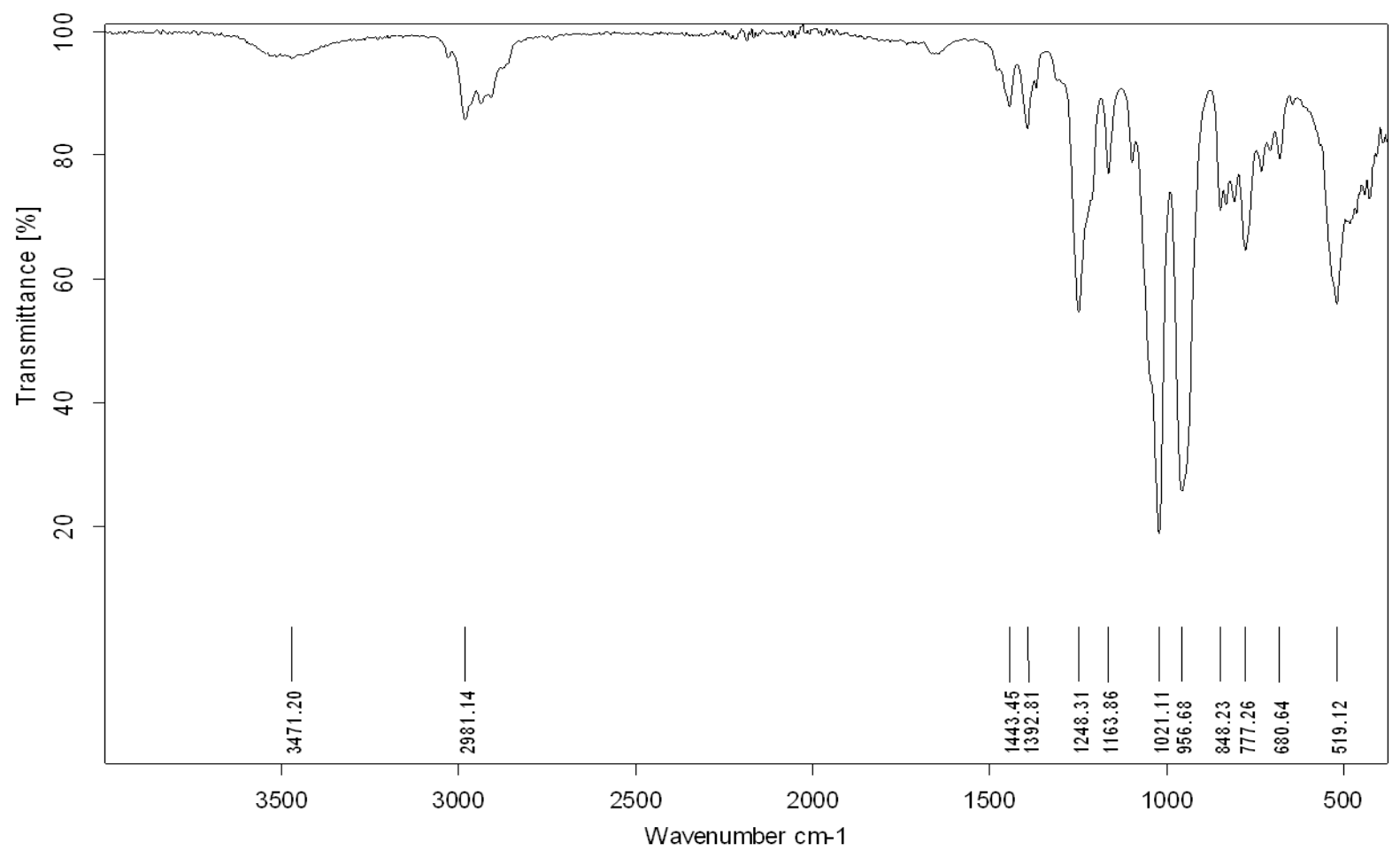


${ }^{1} \mathrm{H}$-NMR (300 MHz), ${ }^{13} \mathrm{C}$-NMR (75 MHz): $\mathrm{CDCl}_{3}$
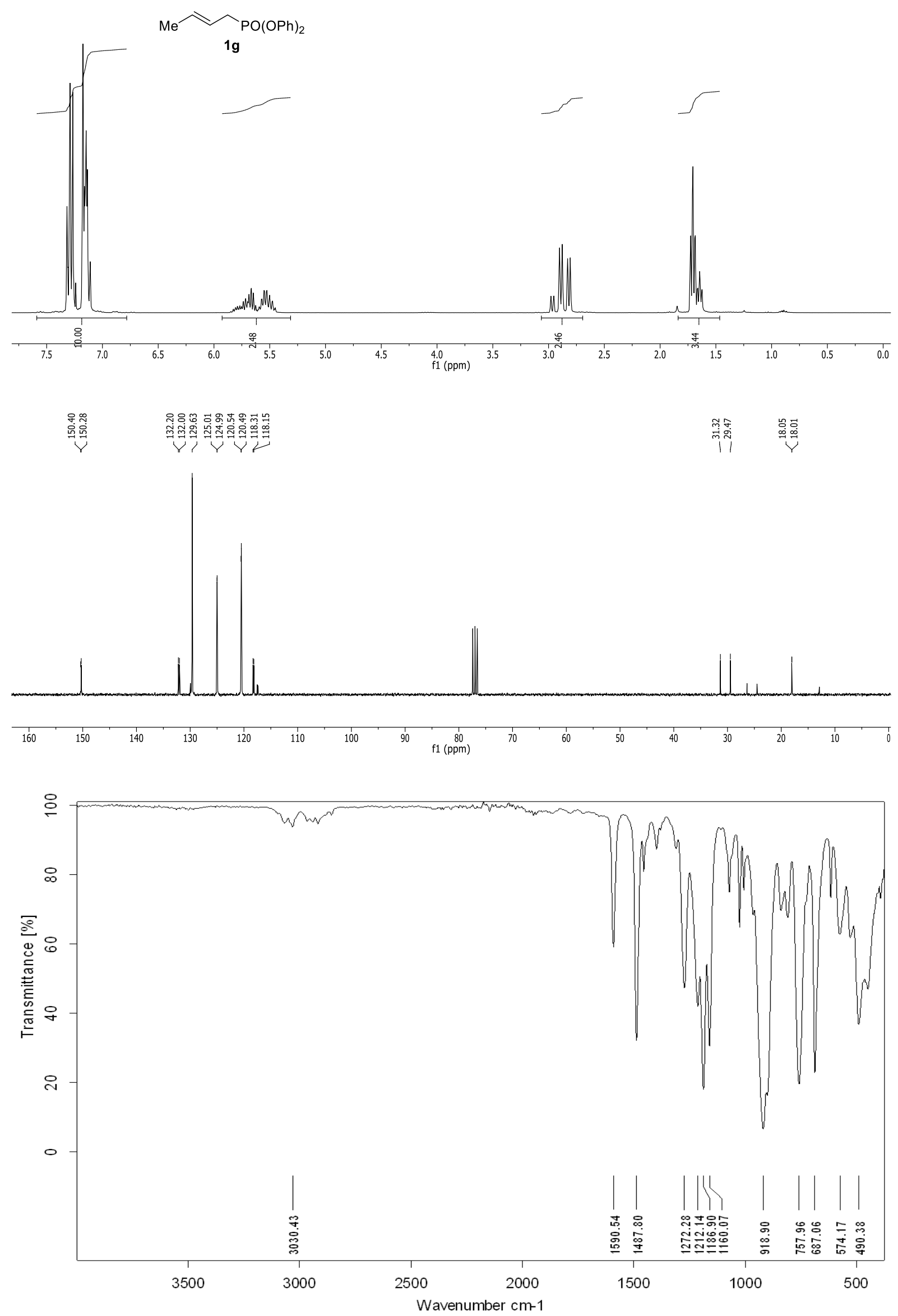
${ }^{1} \mathrm{H}-\mathrm{NMR}(500 \mathrm{MHz}),{ }^{13} \mathrm{C}$-NMR (126 MHz): $\mathrm{CDCl}_{3}$
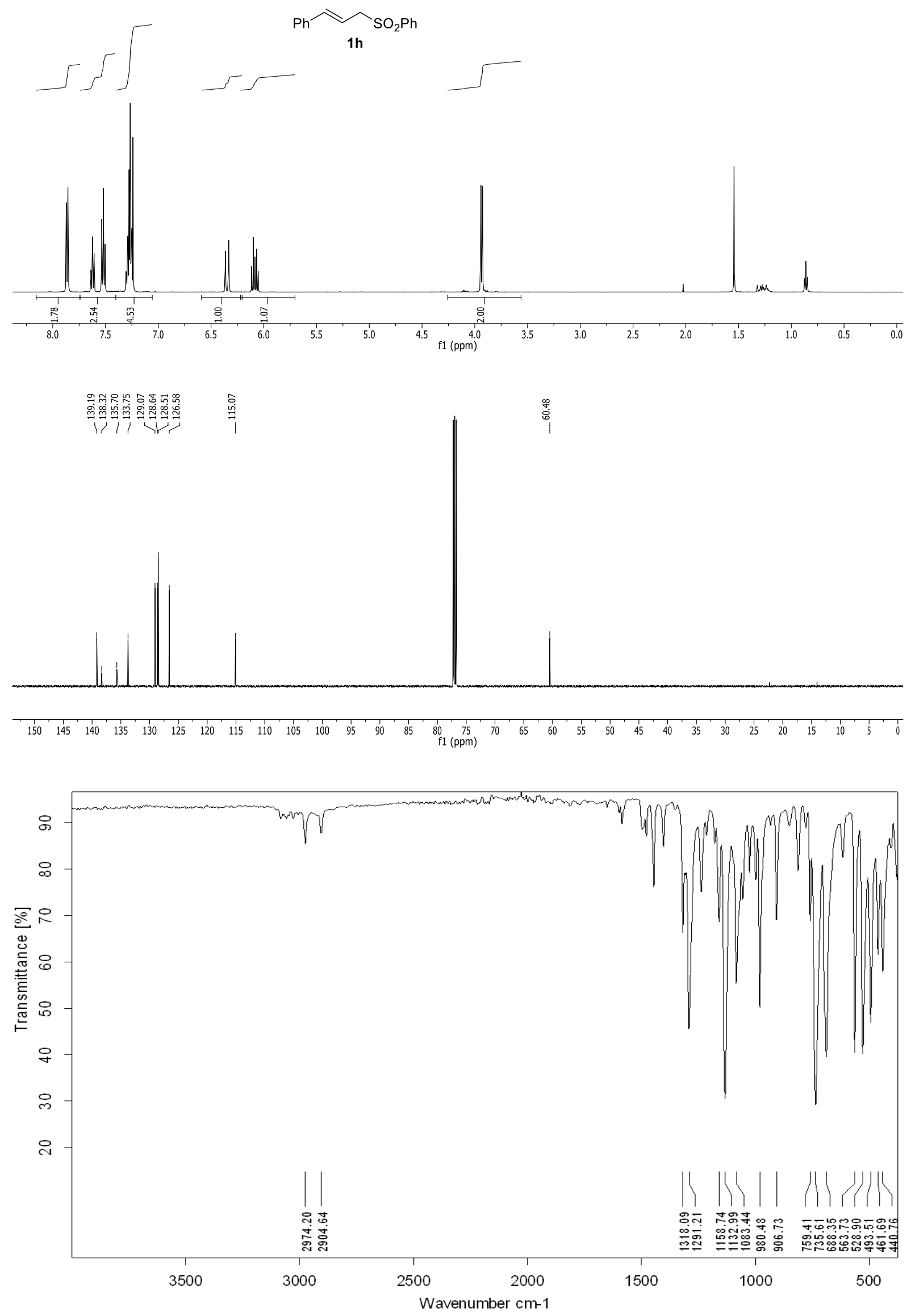
${ }^{1} \mathrm{H}-\mathrm{NMR}(500 \mathrm{MHz}),{ }^{13} \mathrm{C}$-NMR (126 MHz): $\mathrm{CDCl}_{3}$

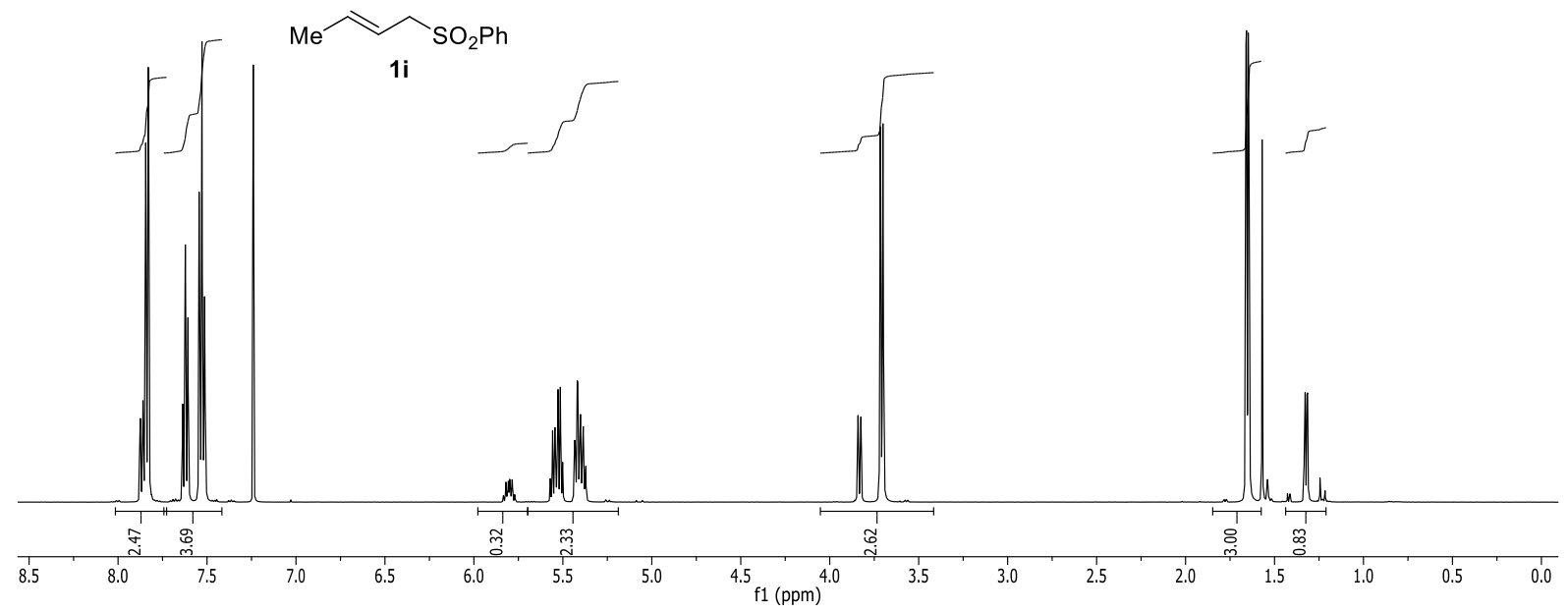

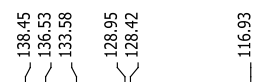
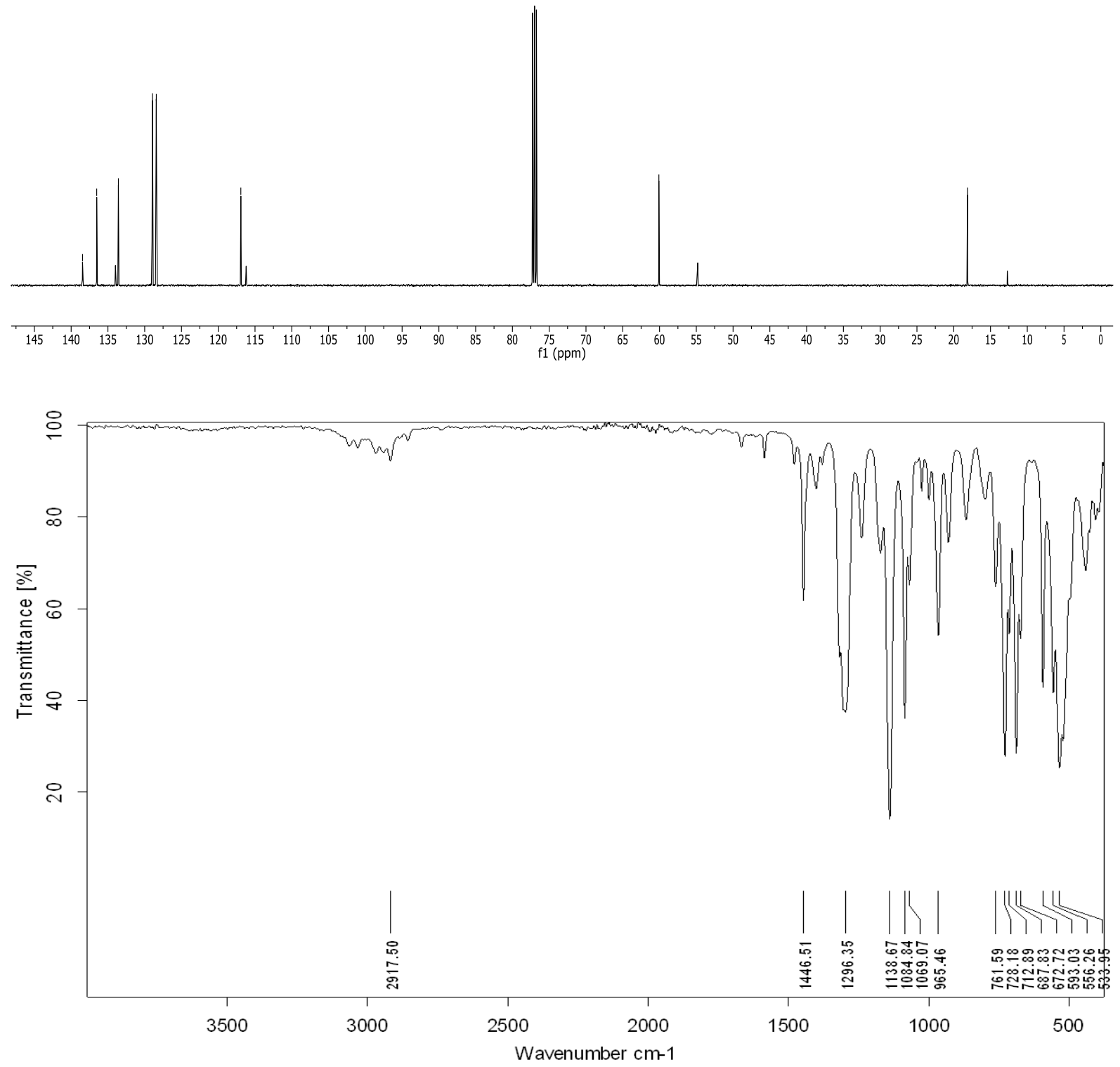
${ }^{1} \mathrm{H}-\mathrm{NMR}(300 \mathrm{MHz}),{ }^{13} \mathrm{C}$-NMR (126 MHz): $\mathrm{CDCl}_{3}$

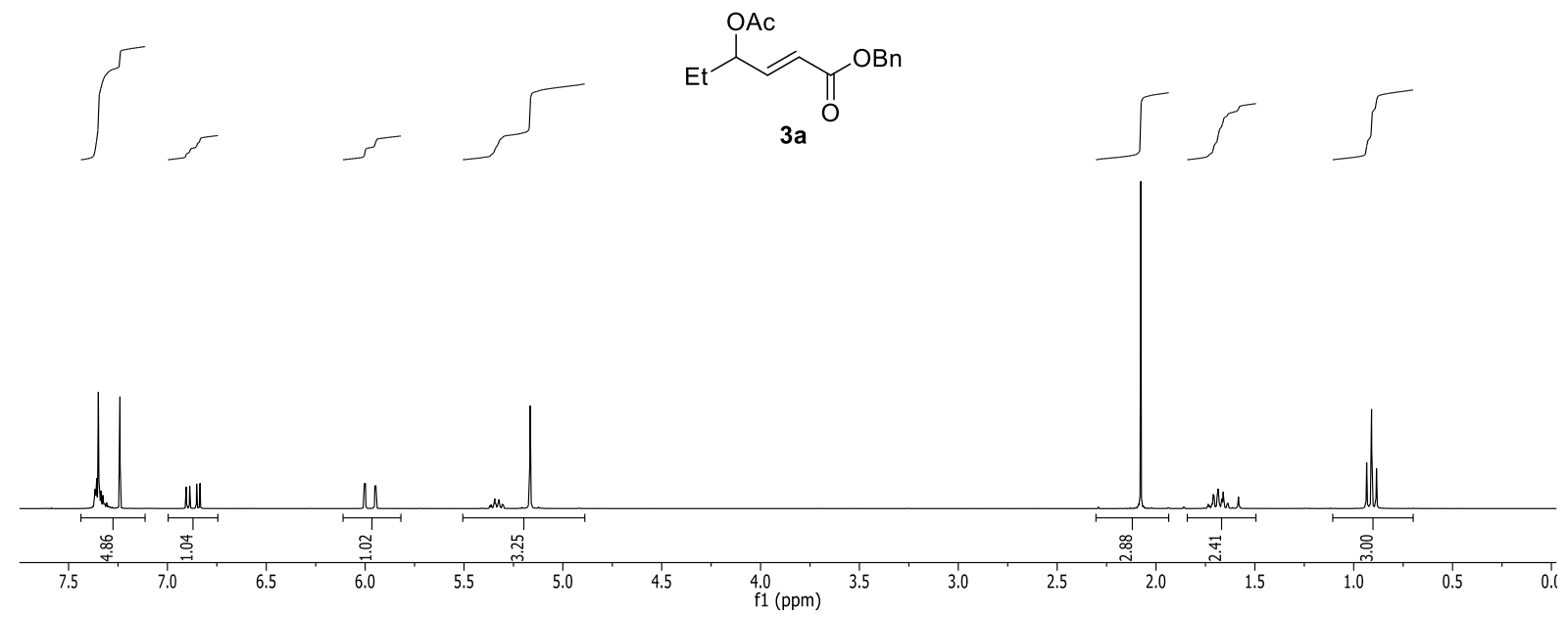

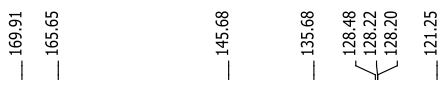

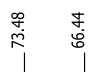

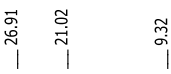
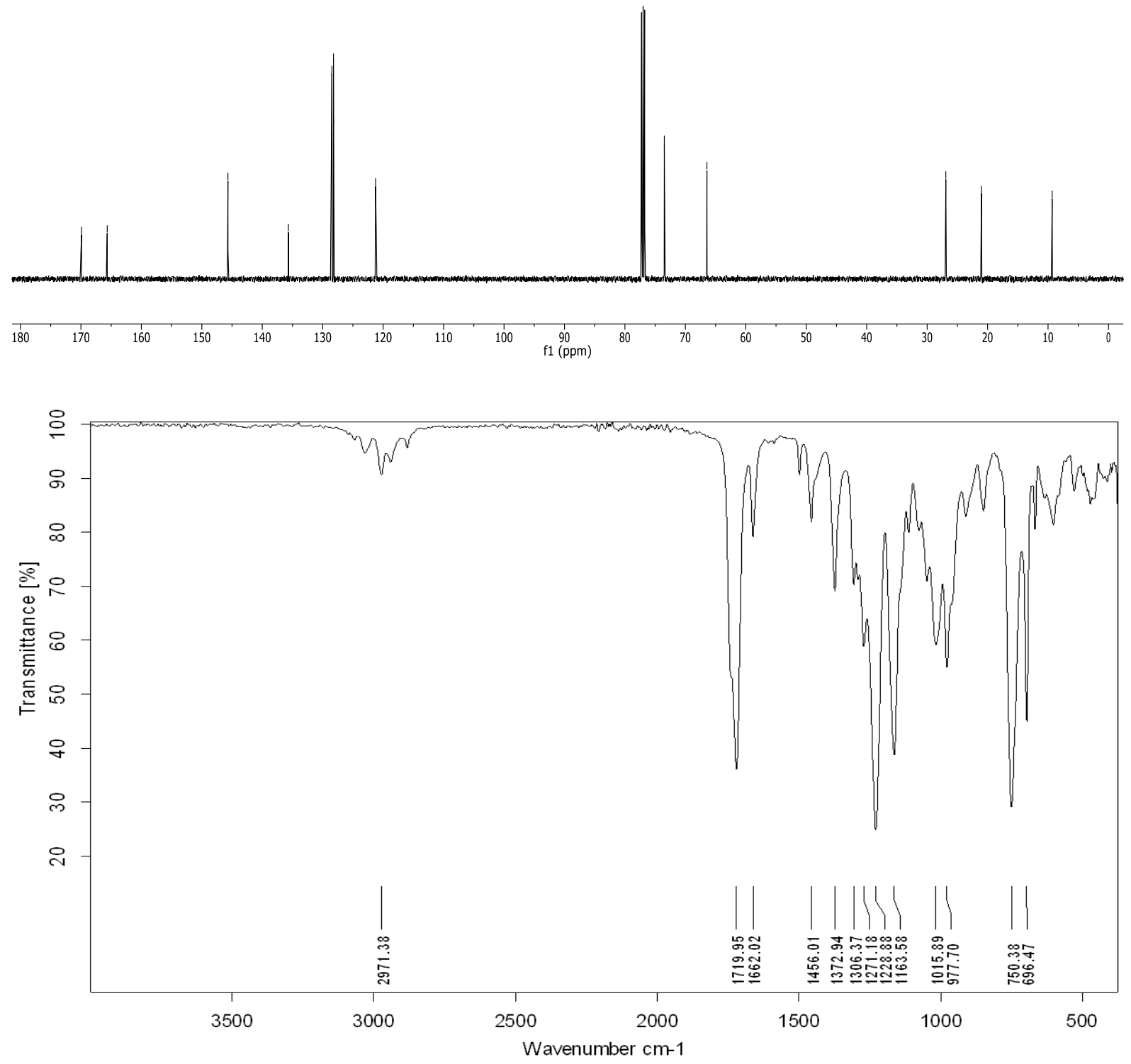
${ }^{1} \mathrm{H}-\mathrm{NMR}(400 \mathrm{MHz}),{ }^{13} \mathrm{C}$-NMR (101 MHz): $\mathrm{CDCl}_{3}$
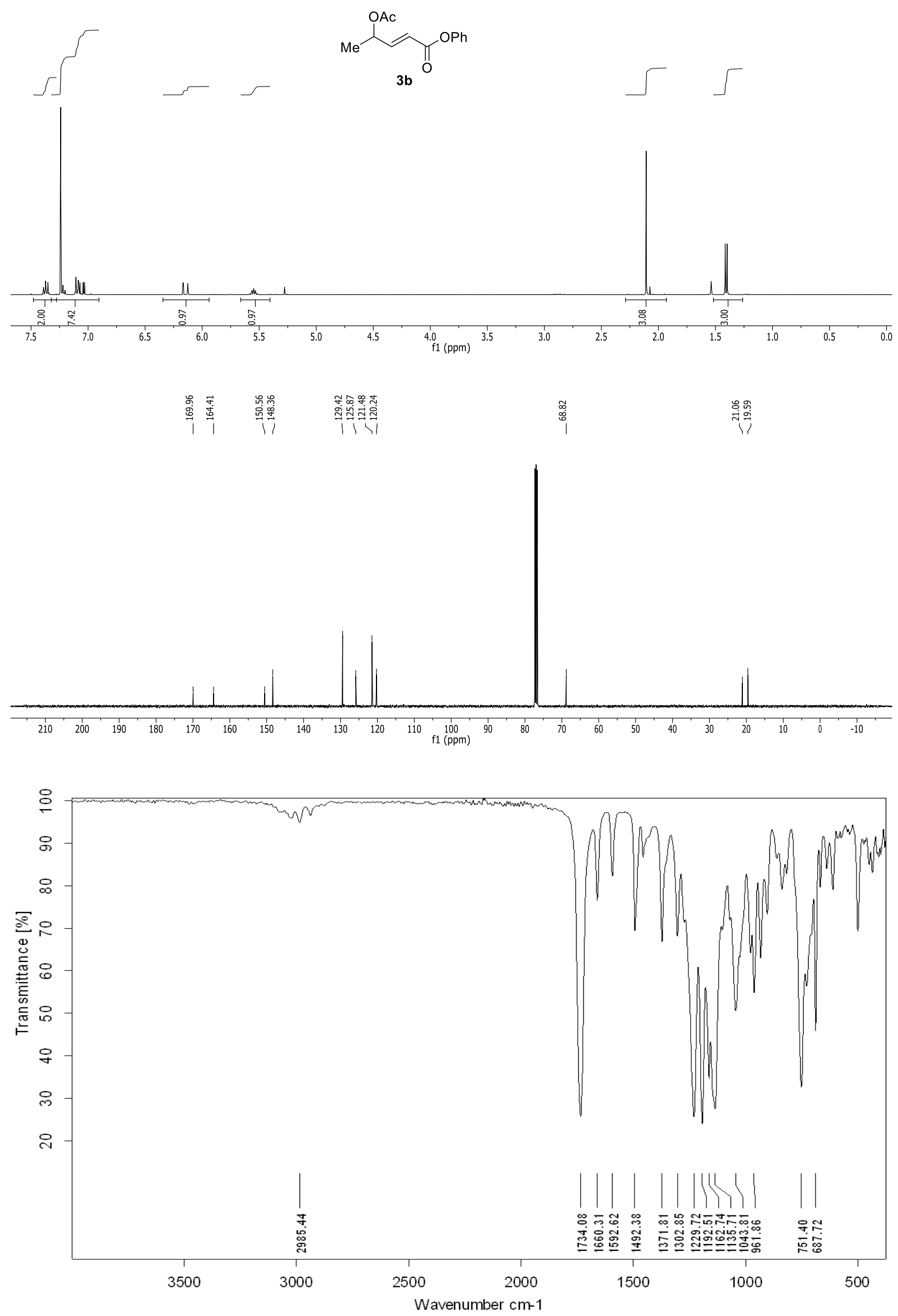
${ }^{1} \mathrm{H}-\mathrm{NMR}(300 \mathrm{MHz}),{ }^{13} \mathrm{C}-\mathrm{NMR}(126 \mathrm{MHz}): \mathrm{CDCl}_{3}$<smiles>CCCCCCOC(=O)/C=C/C(CC)OC(C)=O</smiles>

$3 c$

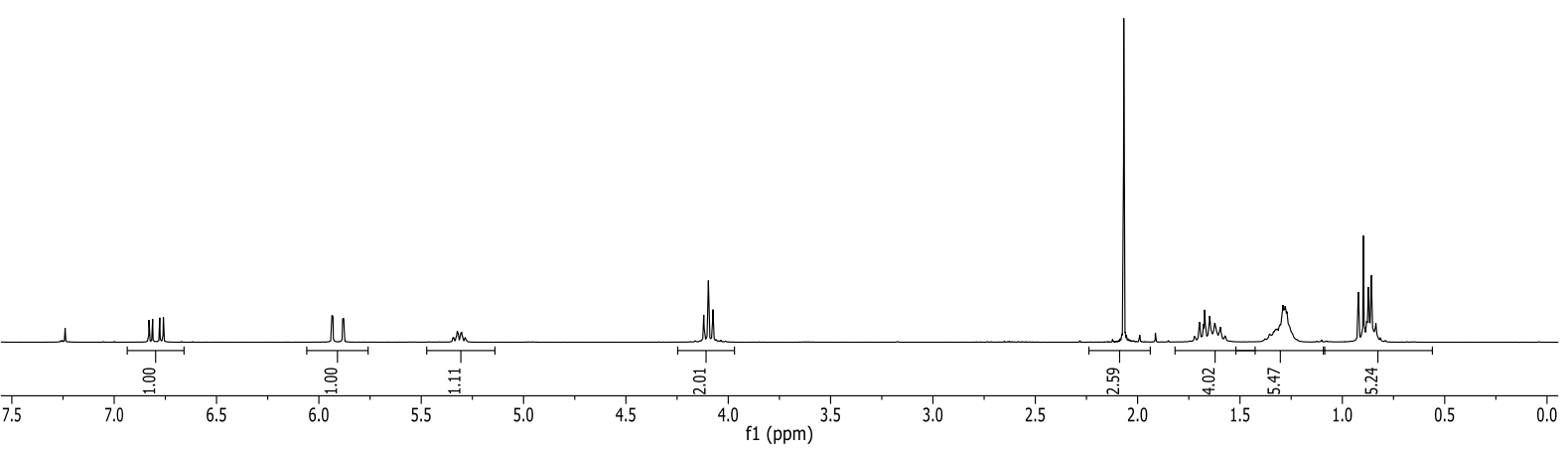

量

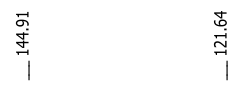

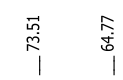

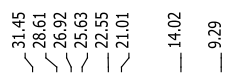

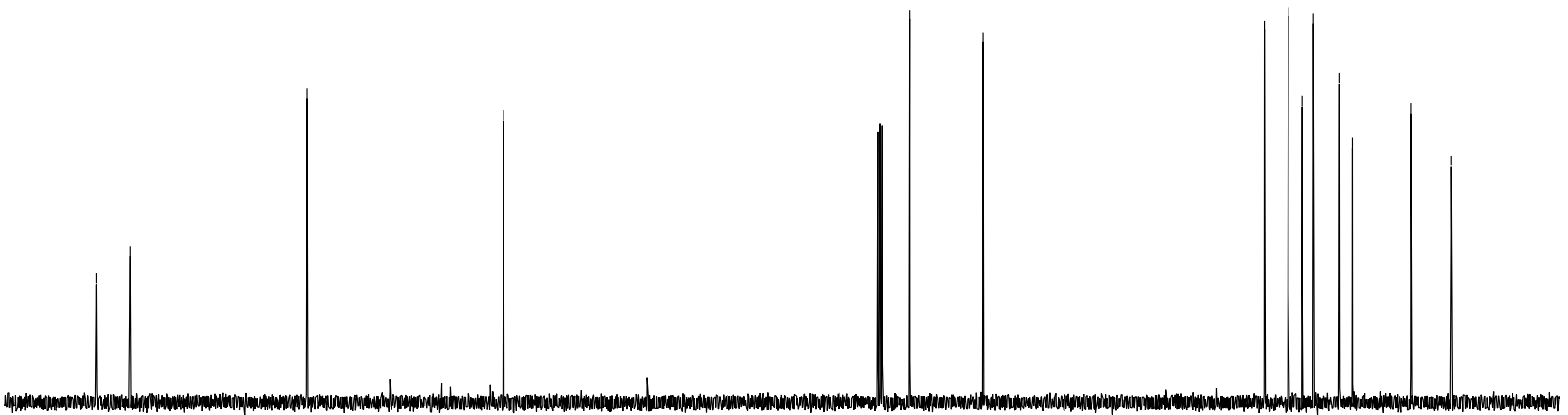

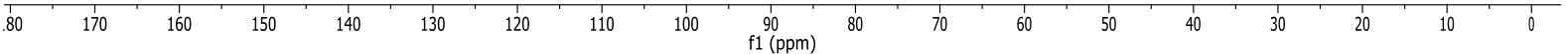

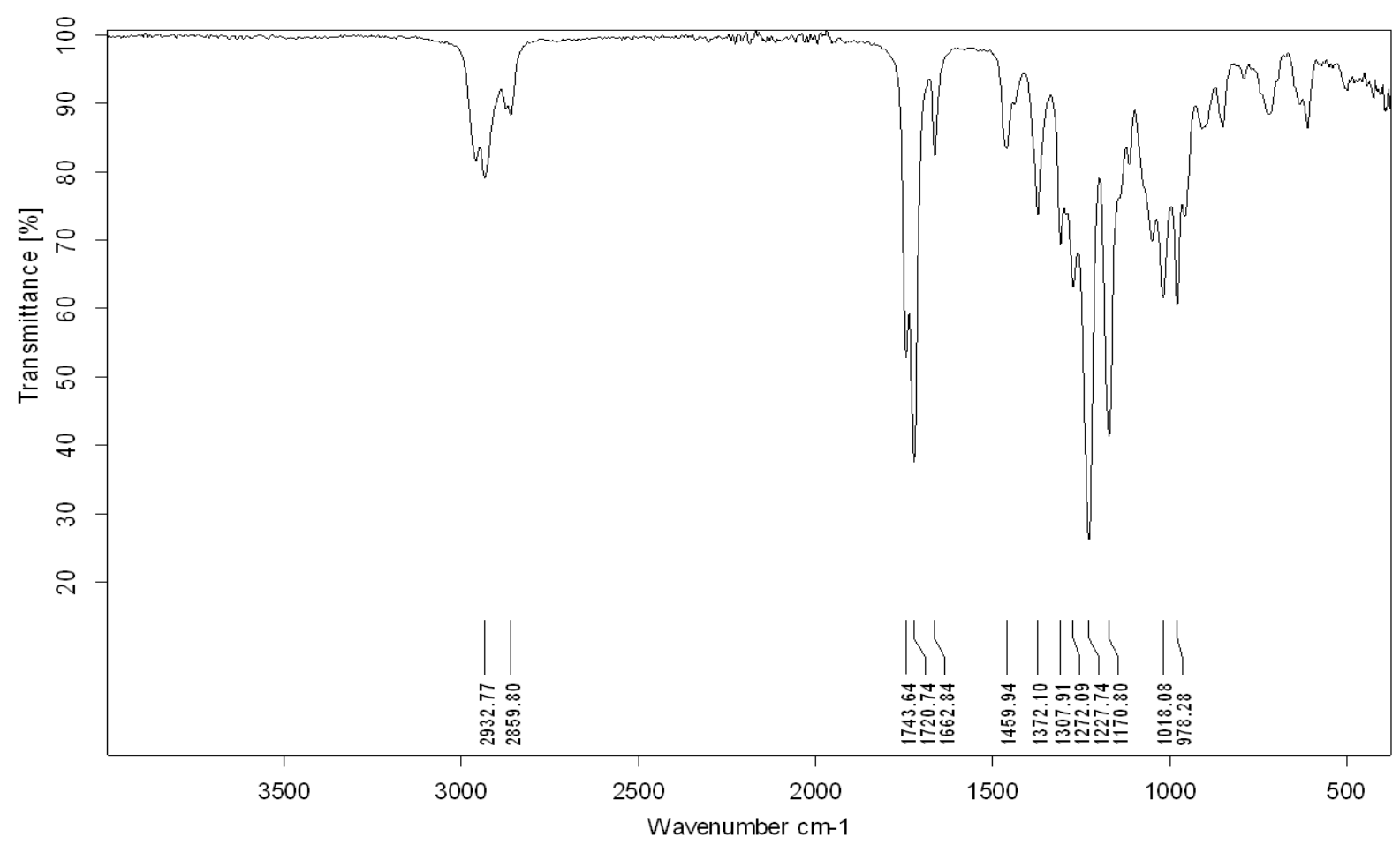


${ }^{1} \mathrm{H}-\mathrm{NMR}(400 \mathrm{MHz}),{ }^{13} \mathrm{C}$-NMR (101 MHz): $\mathrm{CDCl}_{3}$
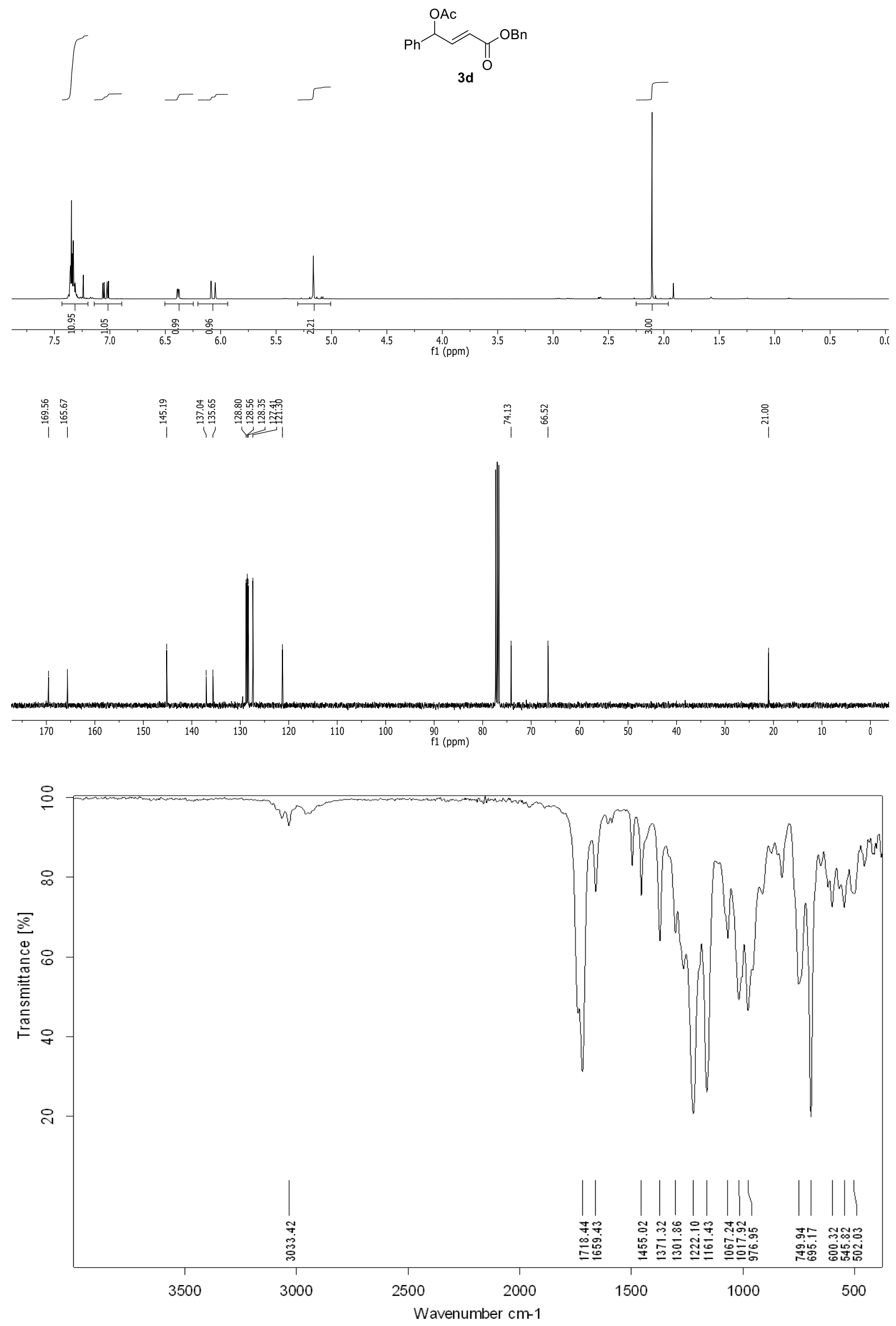
${ }^{1} \mathrm{H}-\mathrm{NMR}(400 \mathrm{MHz}),{ }^{13} \mathrm{C}$-NMR (101 MHz): $\mathrm{CDCl}_{3}$

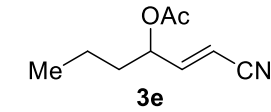

$\sqrt{5}$
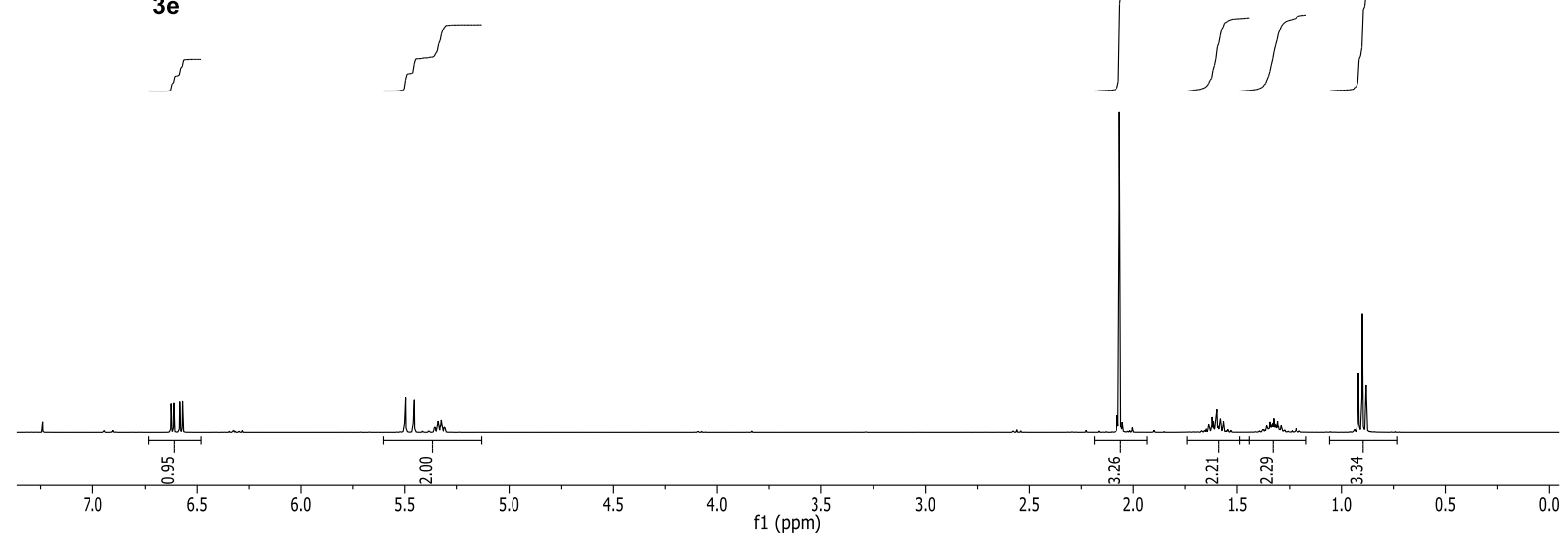

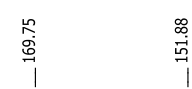

|

$\stackrel{8}{\stackrel{8}{1}}$

帛
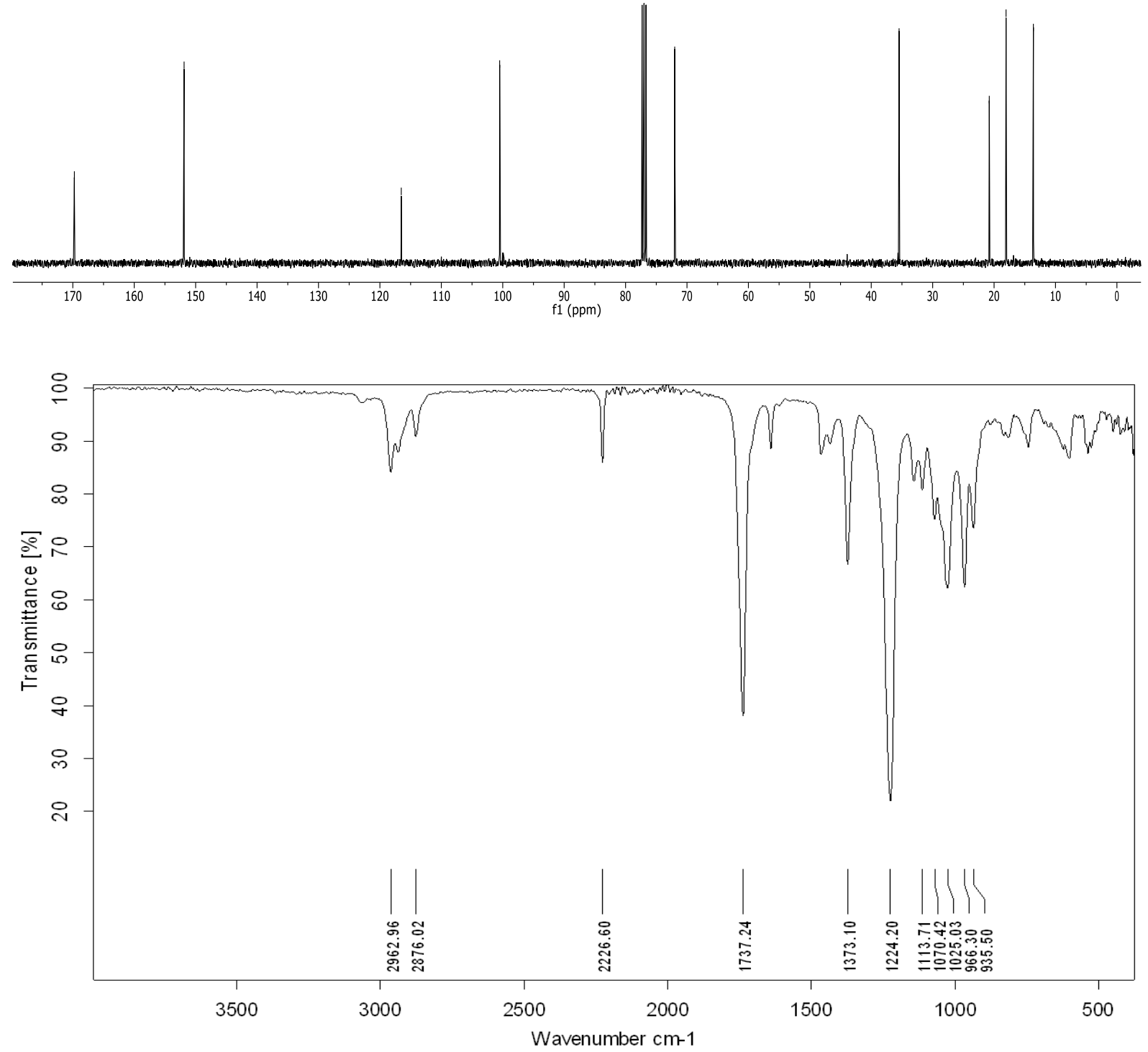
${ }^{1} \mathrm{H}-\mathrm{NMR}(300 \mathrm{MHz}),{ }^{13} \mathrm{C}-\mathrm{NMR}(126 \mathrm{MHz}): \mathrm{CDCl}_{3}$<smiles>CCOC(C)C(C)C=[Pb]([Hg])OCC</smiles>
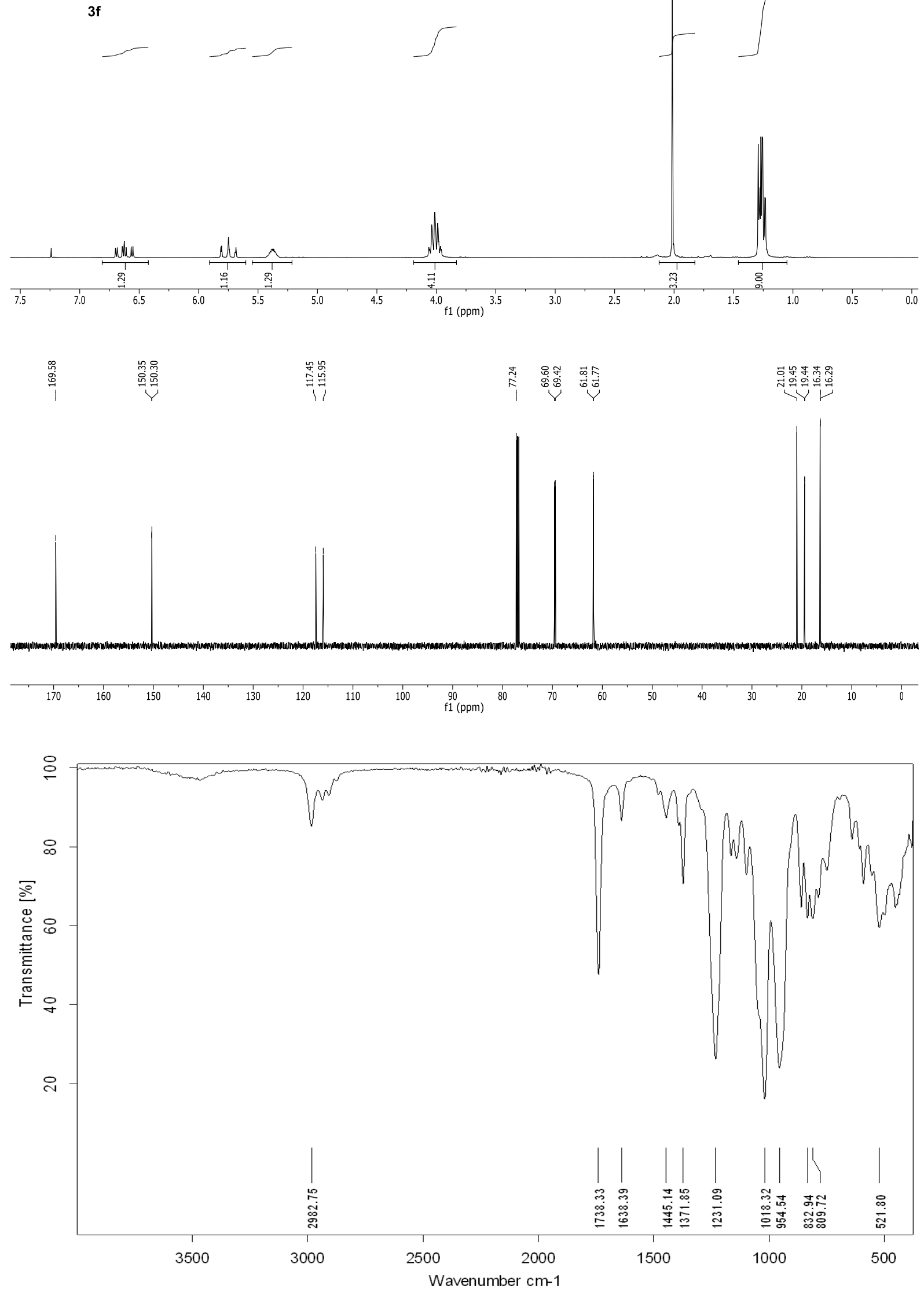
${ }^{1} \mathrm{H}-\mathrm{NMR}(300 \mathrm{MHz}),{ }^{13} \mathrm{C}-\mathrm{NMR}(75 \mathrm{MHz}): \mathrm{CDCl}_{3}$
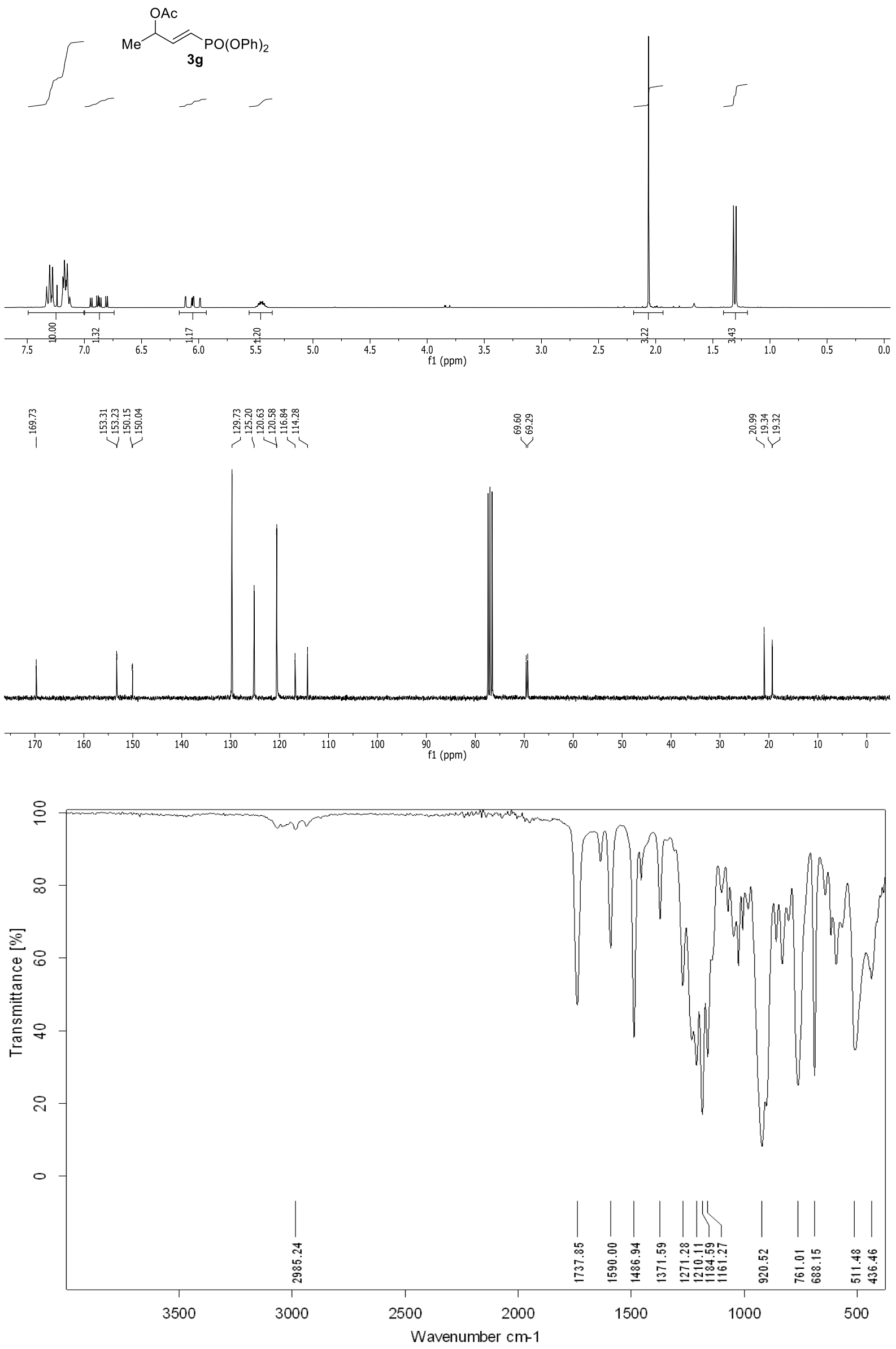
${ }^{1} \mathrm{H}$-NMR (300 MHz), ${ }^{13}$ C-NMR (126 MHz): $\mathrm{CDCl}_{3}$
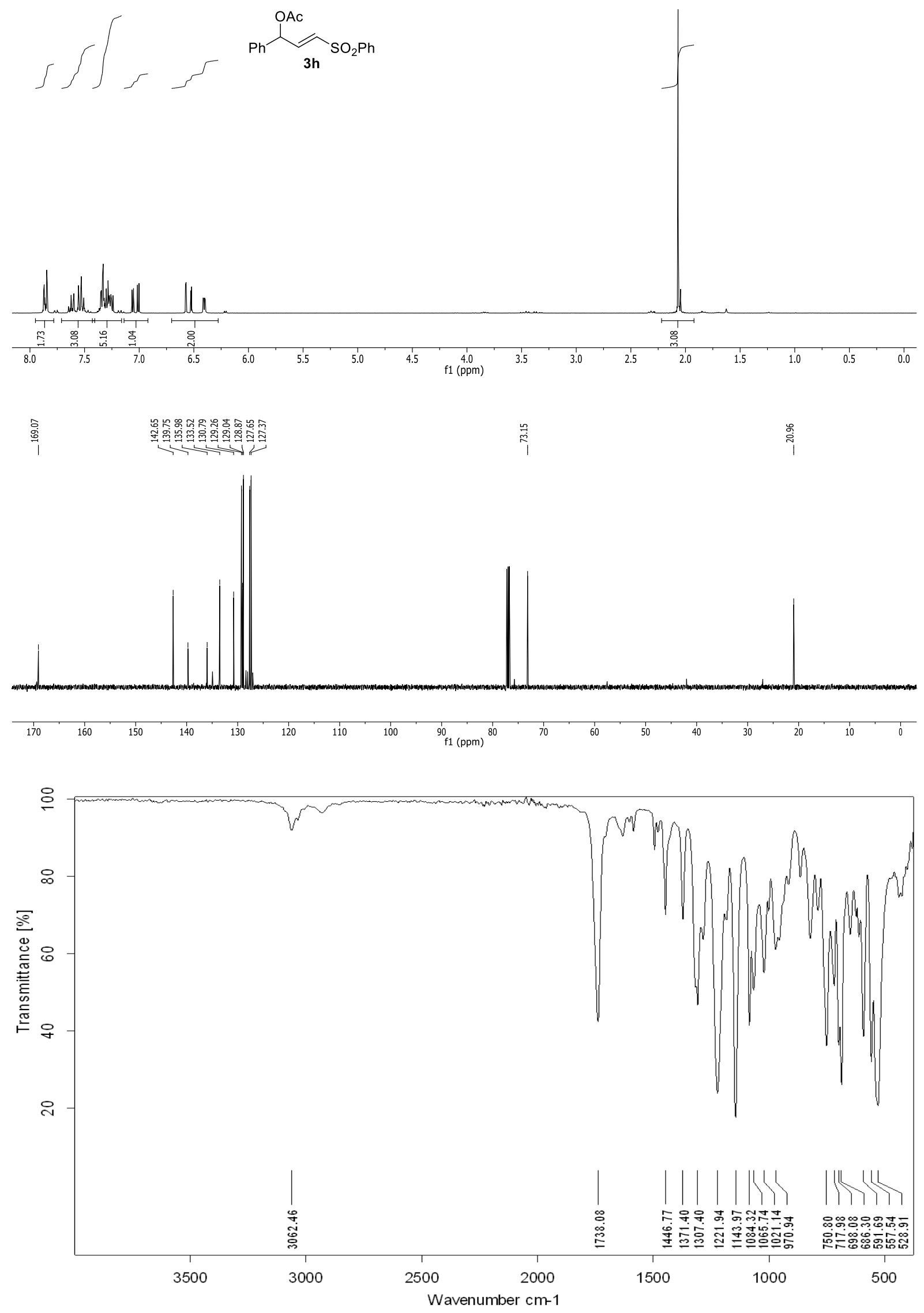
${ }^{1} \mathrm{H}-\mathrm{NMR}(300 \mathrm{MHz}),{ }^{13} \mathrm{C}-\mathrm{NMR}(75 \mathrm{MHz}): \mathrm{CDCl}_{3}$

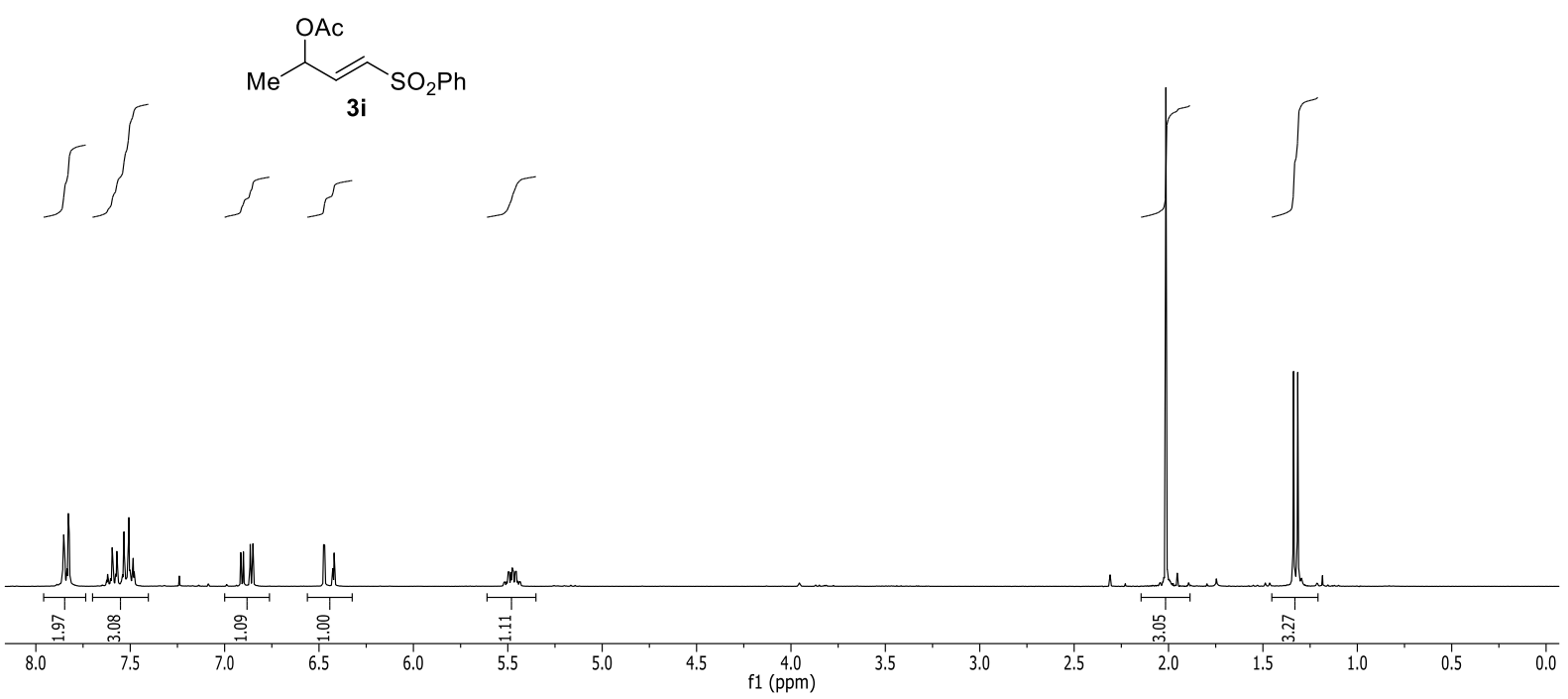

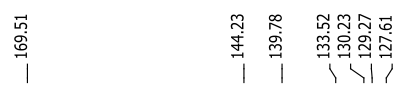

$\stackrel{\infty}{\stackrel{\infty}{\infty}}$

i̊m
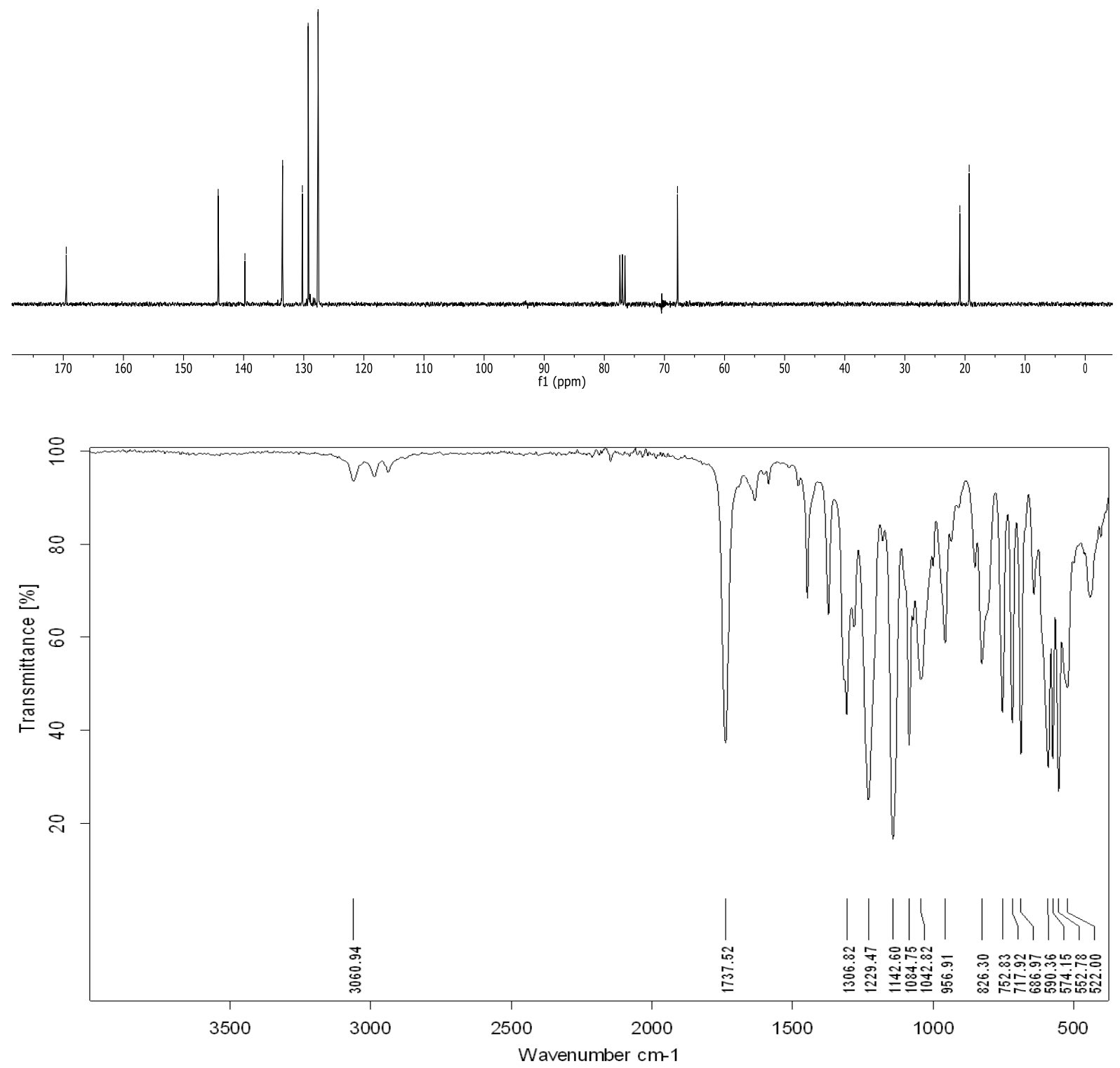

S46 
${ }^{1} \mathrm{H}-\mathrm{NMR}(300 \mathrm{MHz}),{ }^{13} \mathrm{C}-\mathrm{NMR}(75 \mathrm{MHz}): \mathrm{CDCl}_{3}$

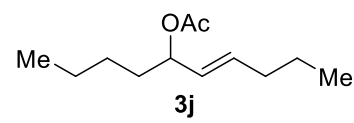

3]
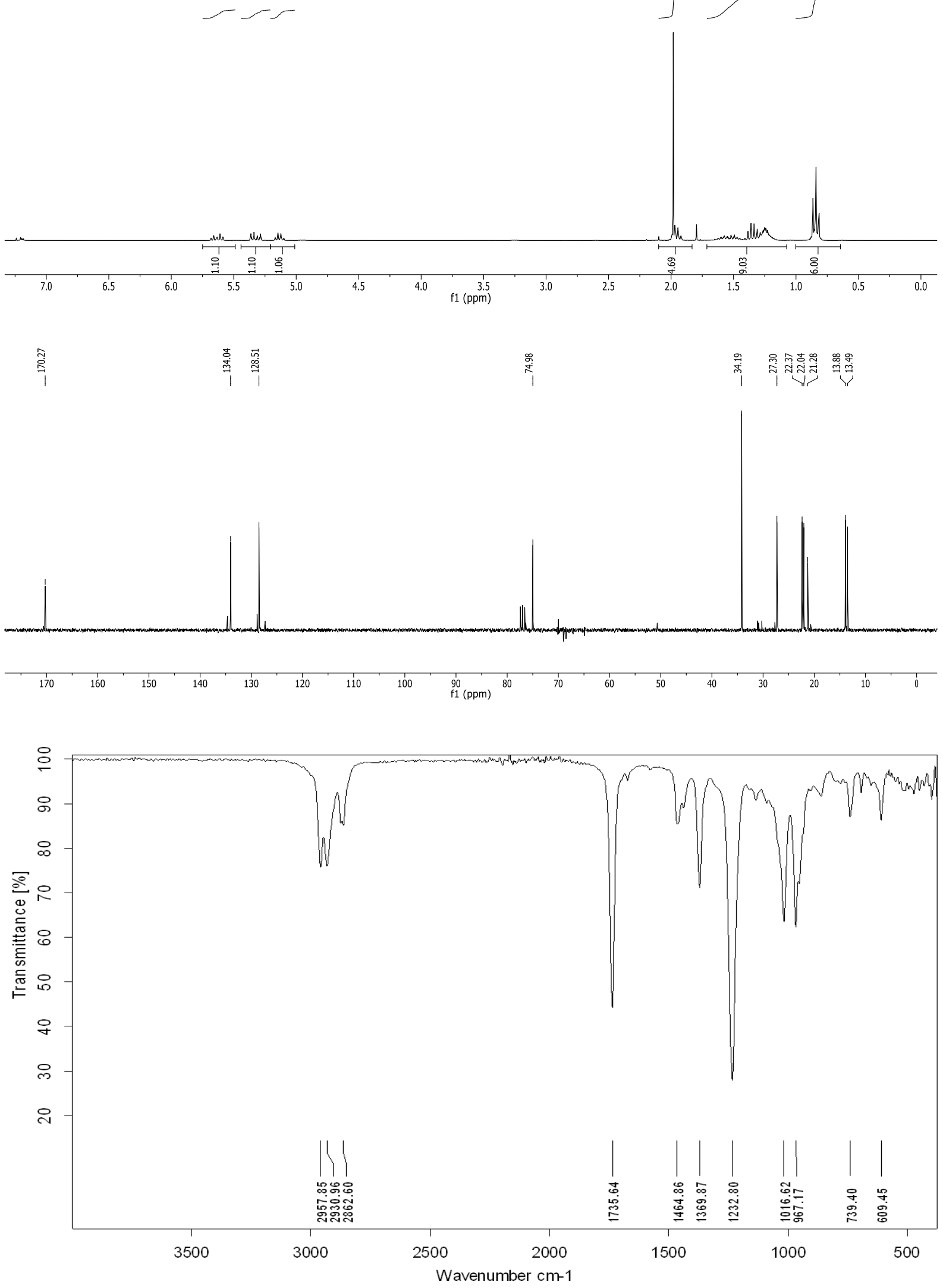
${ }^{1} \mathrm{H}-\mathrm{NMR}(300 \mathrm{MHz}),{ }^{13} \mathrm{C}-\mathrm{NMR}(75 \mathrm{MHz}): \mathrm{CDCl}_{3}$<smiles>CC(=O)OC1C=CCCCCC1</smiles>

3k
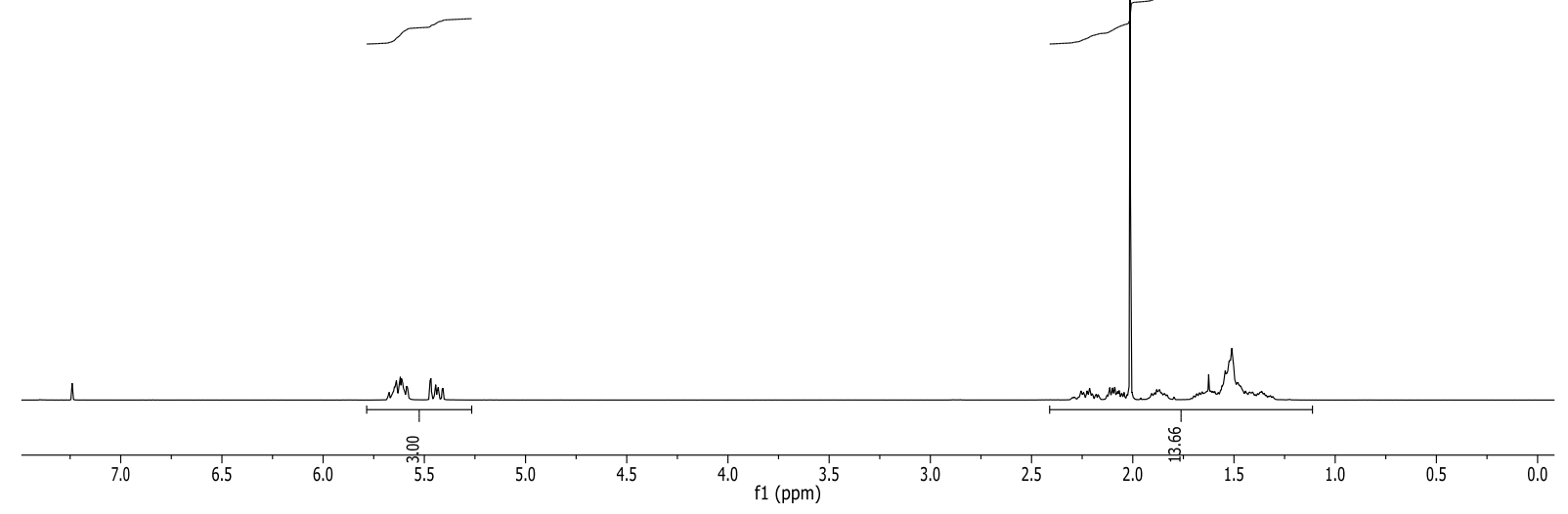

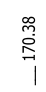

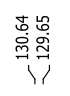

萬

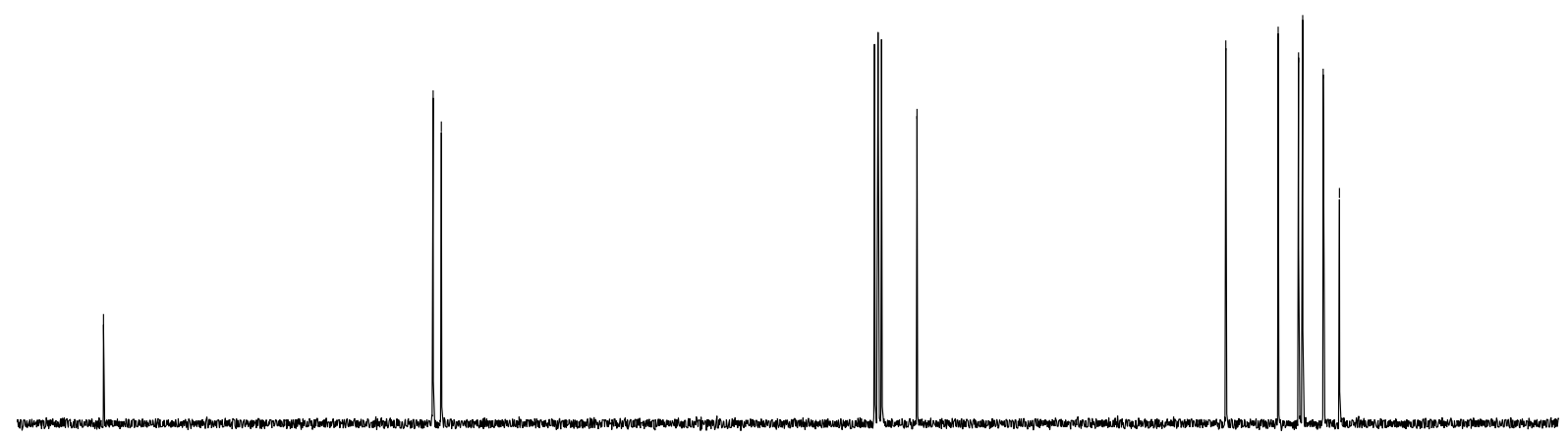

$$
80
$$

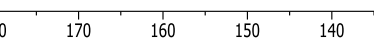

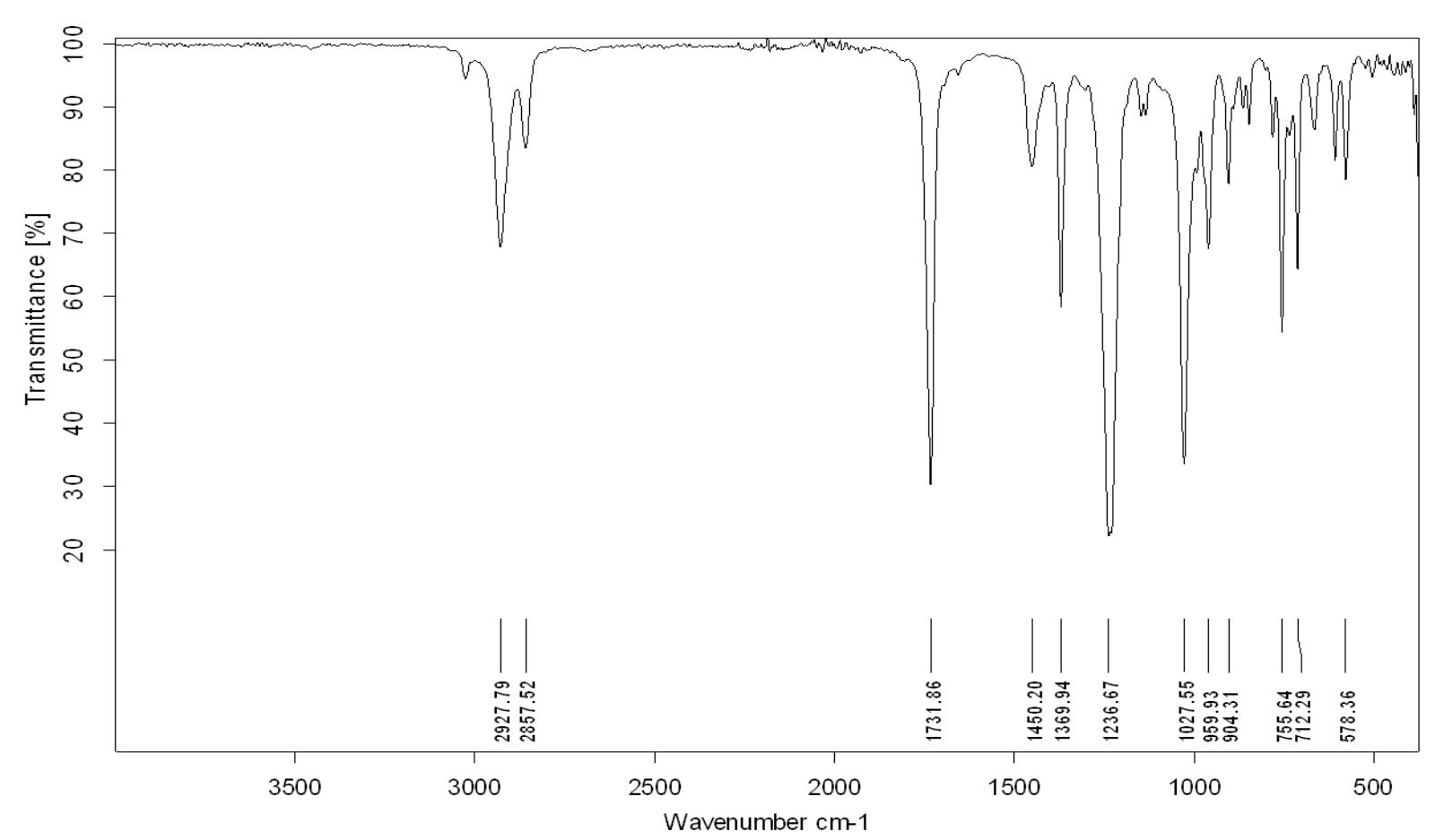


${ }^{1} \mathrm{H}$-NMR (500 MHz), ${ }^{13} \mathrm{C}$-NMR (126 MHz): $\mathrm{CDCl}_{3}$
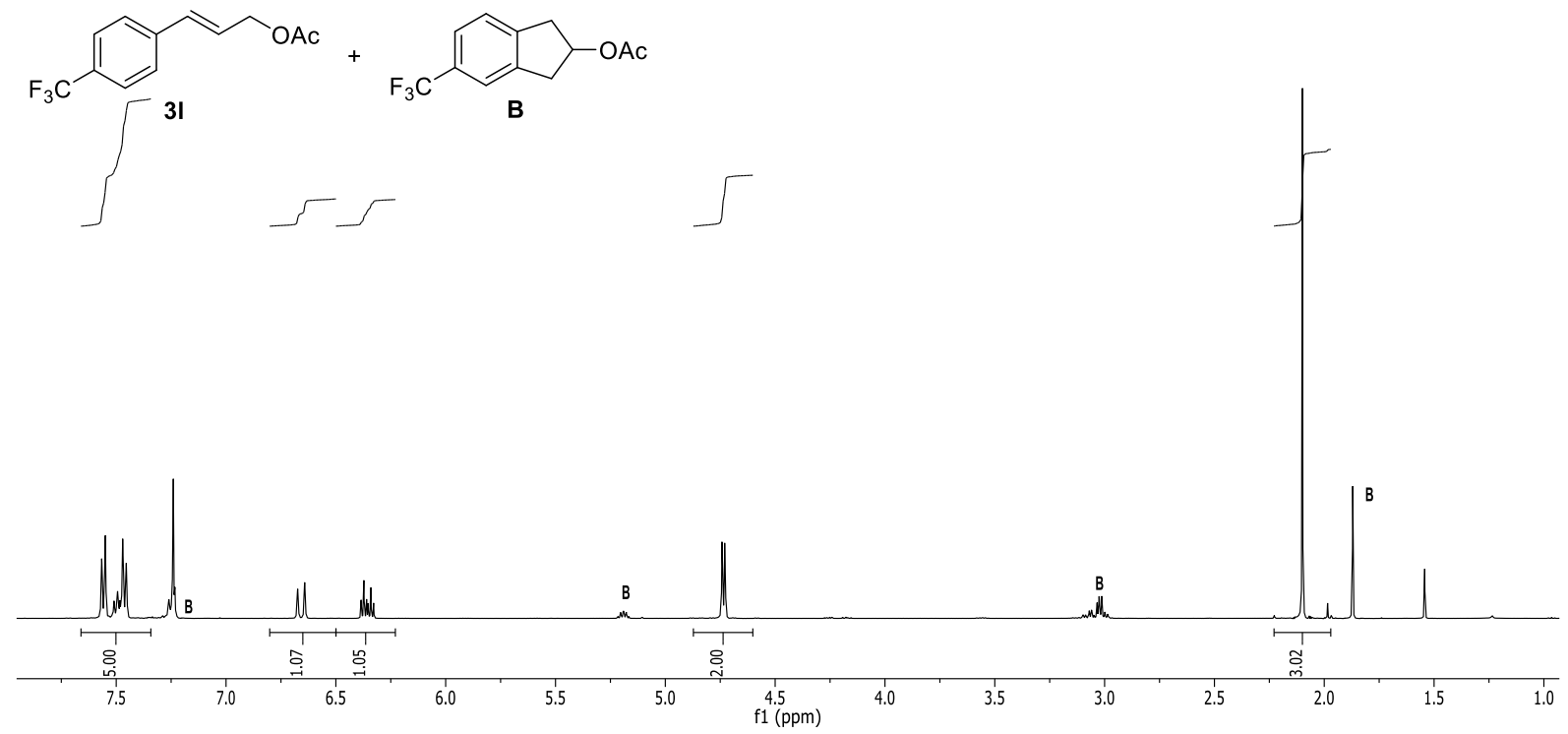

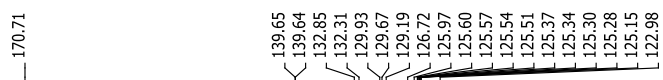
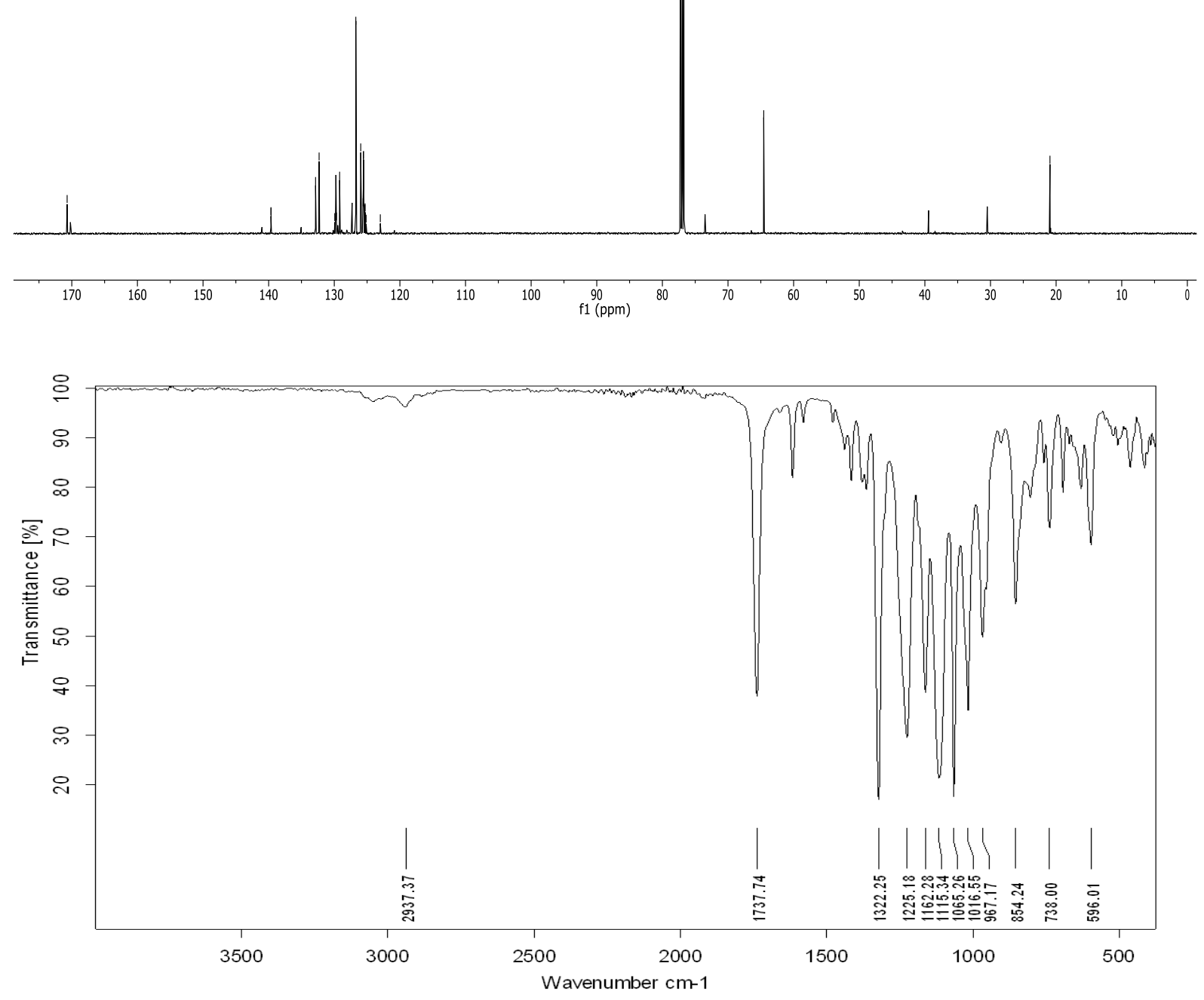
${ }^{1} \mathrm{H}-\mathrm{NMR}(400 \mathrm{MHz}),{ }^{13} \mathrm{C}-\mathrm{NMR}(101 \mathrm{MHz}): \mathrm{CDCl}_{3}$

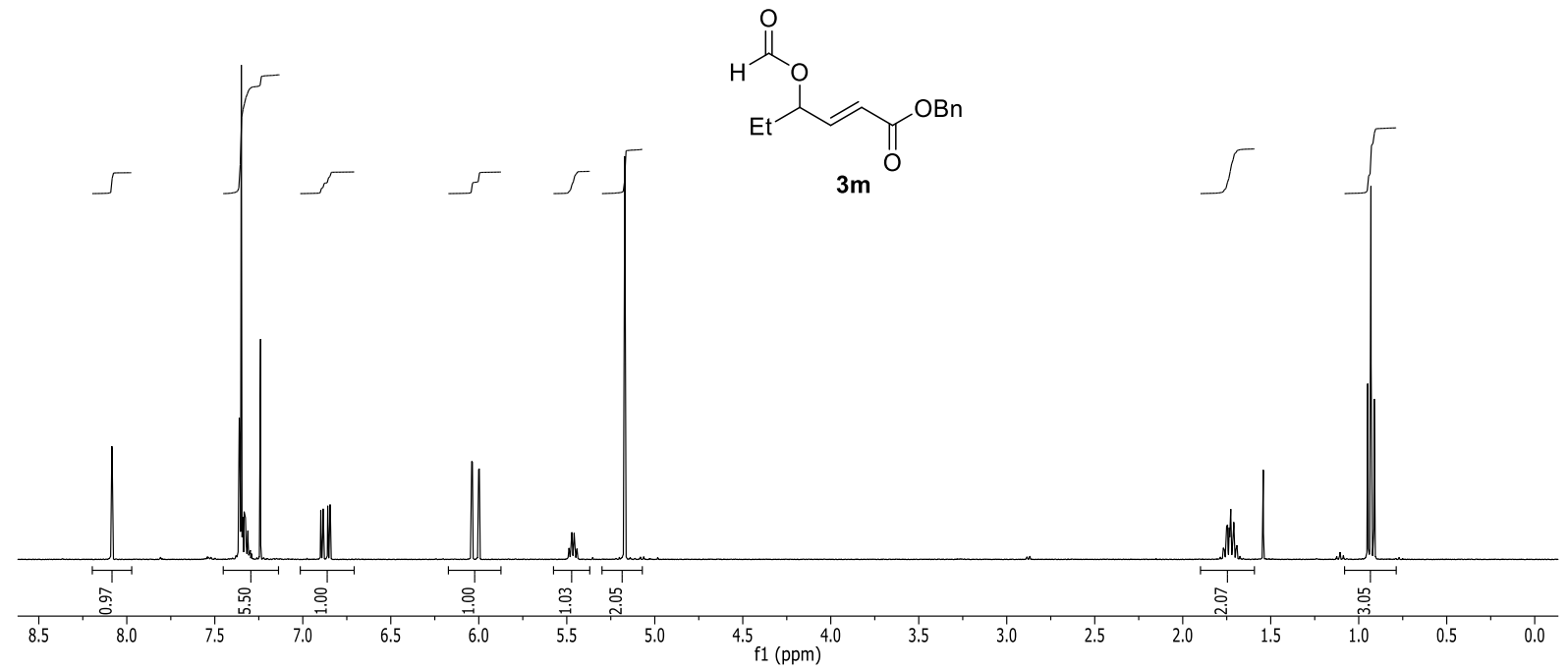

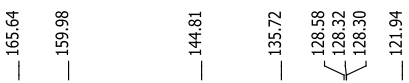

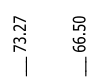

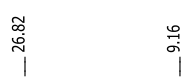
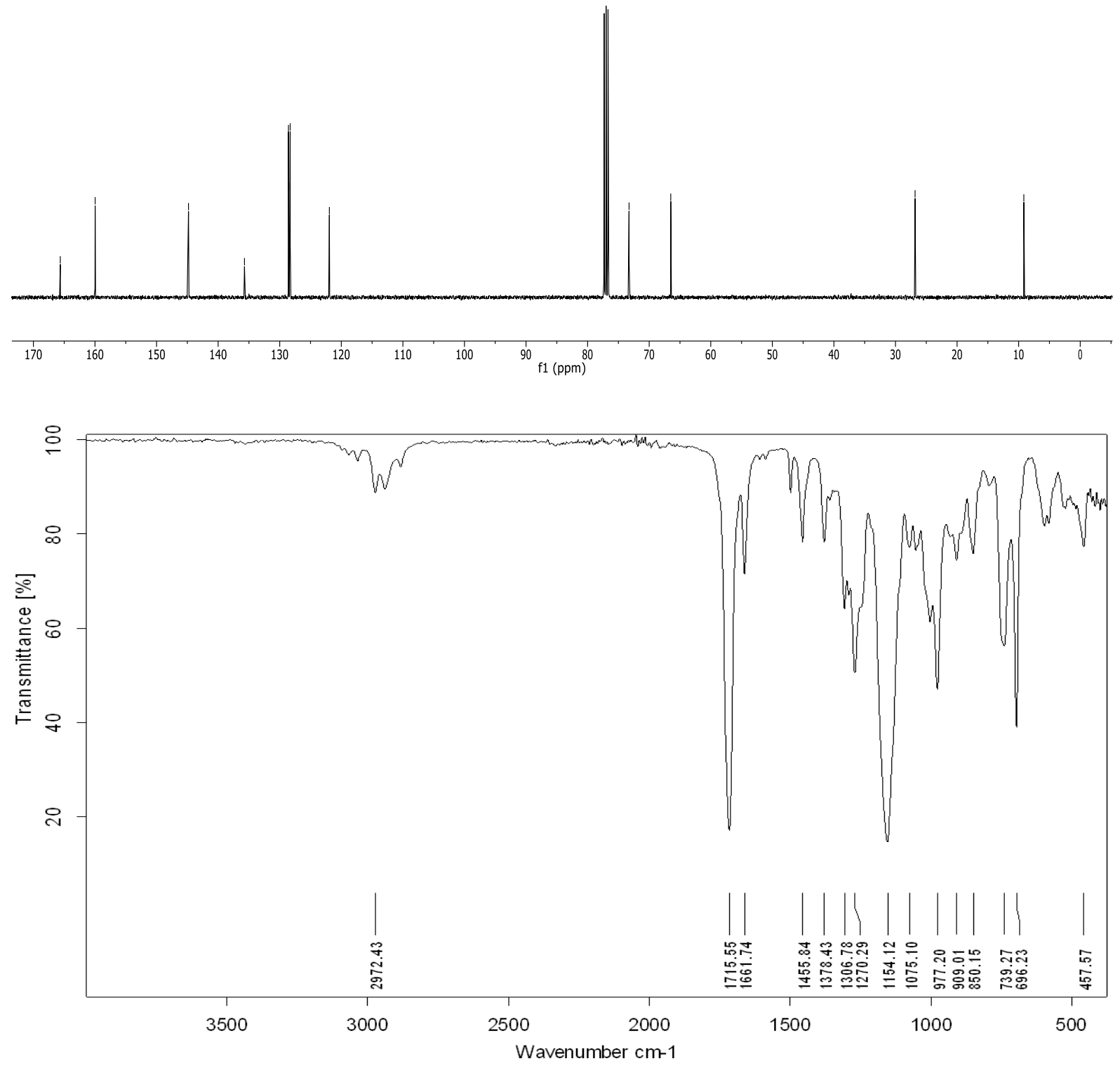
${ }^{1} \mathrm{H}-\mathrm{NMR}(400 \mathrm{MHz}),{ }^{13} \mathrm{C}$-NMR (101 MHz): $\mathrm{CDCl}_{3}$

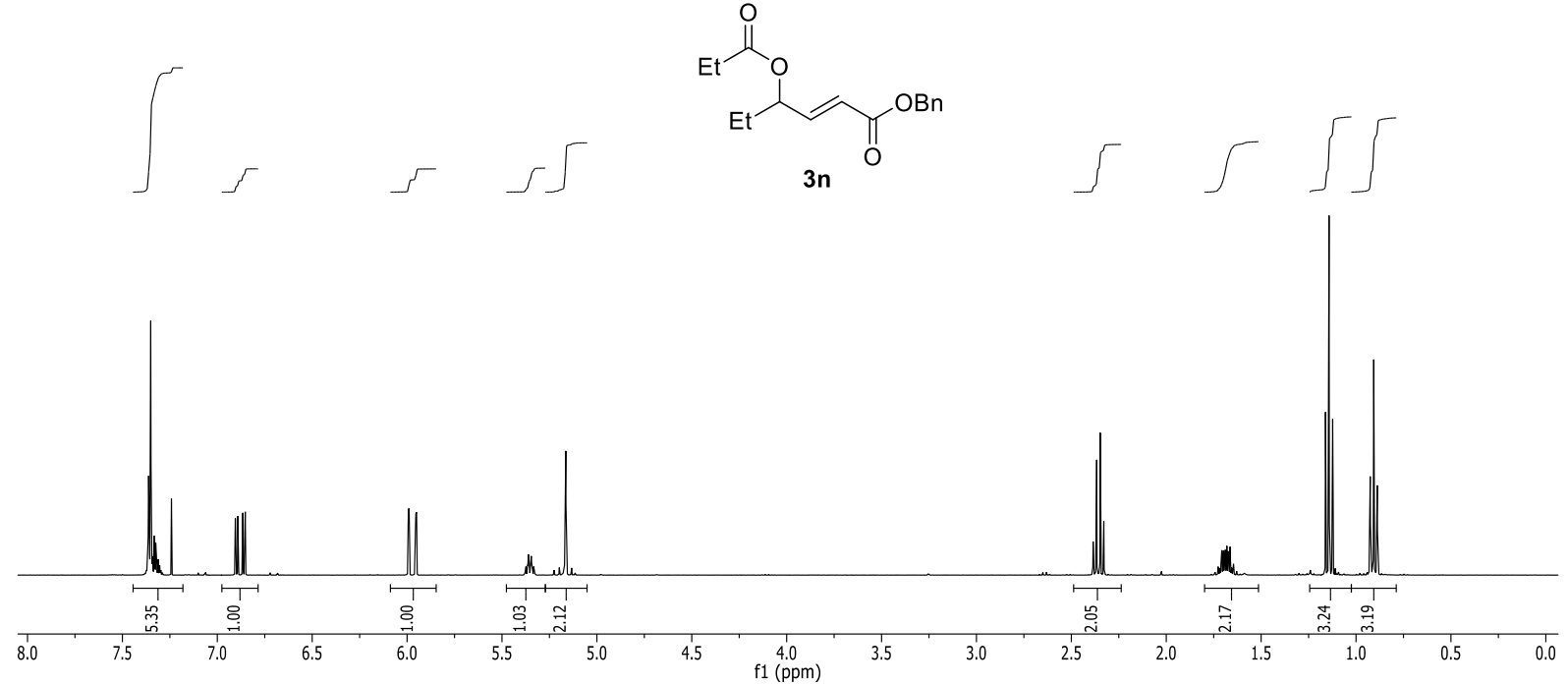

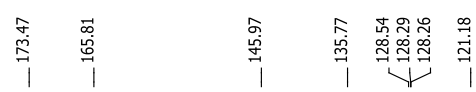

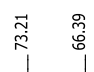

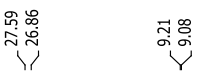

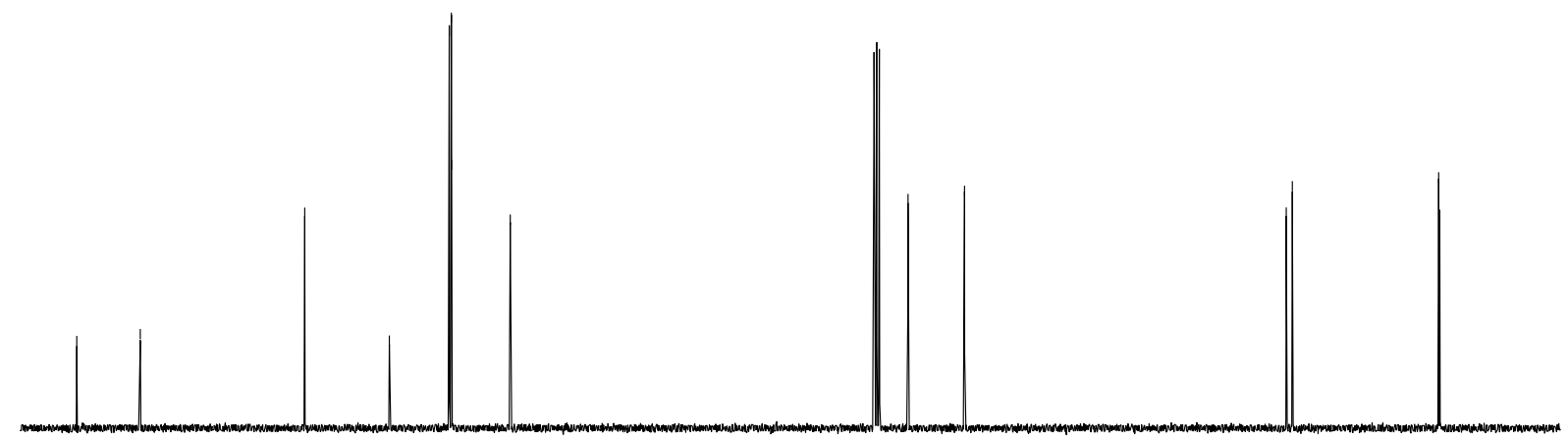

30
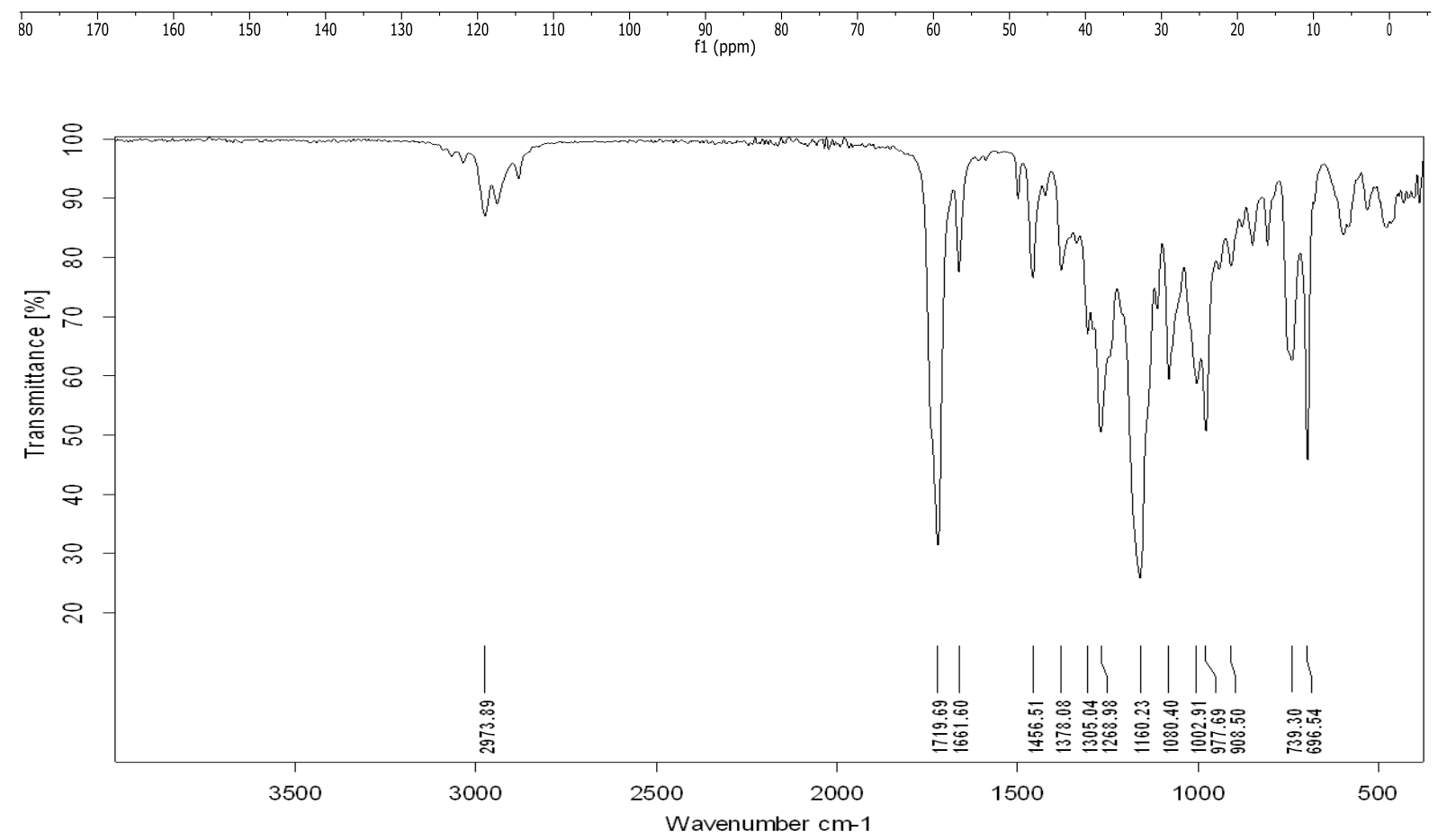
${ }^{1} \mathrm{H}-\mathrm{NMR}(400 \mathrm{MHz}),{ }^{13} \mathrm{C}$-NMR (101 MHz): $\mathrm{CDCl}_{3}$

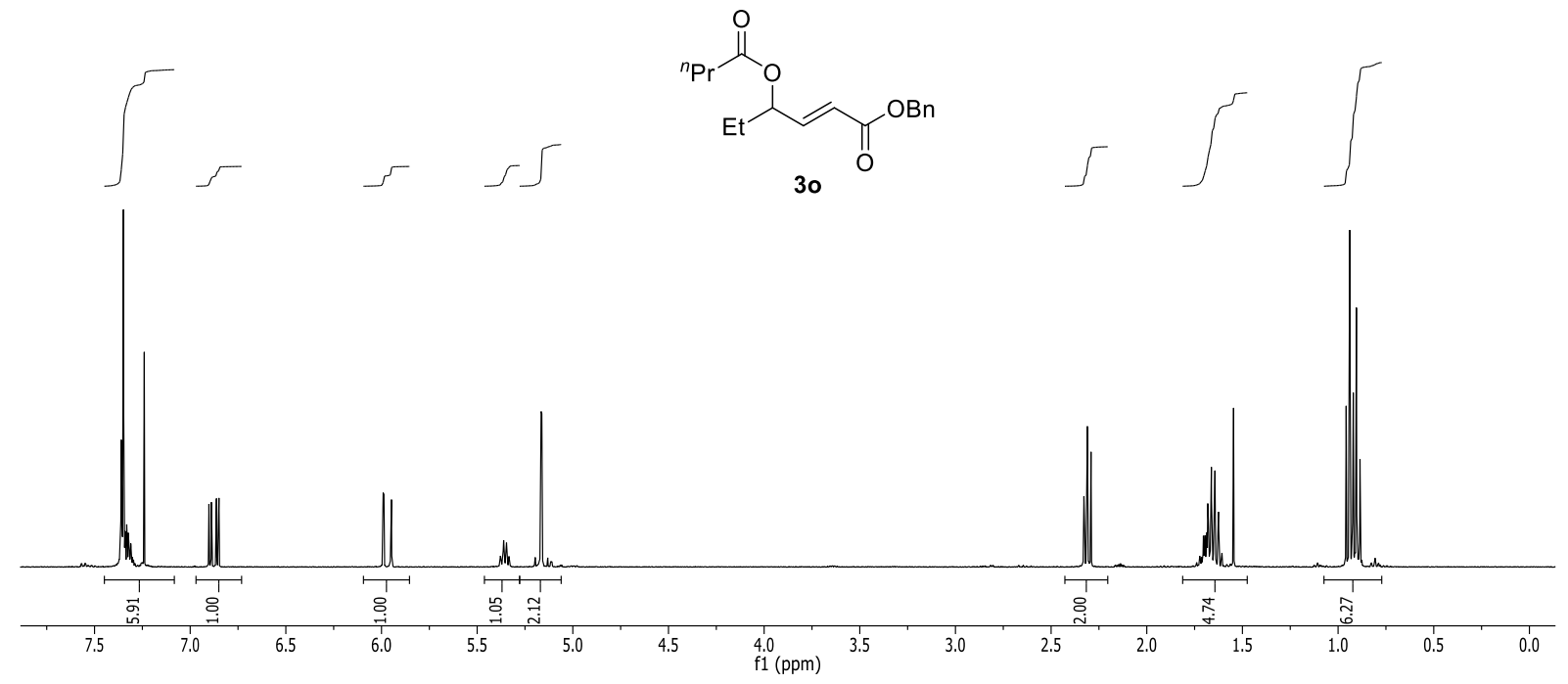

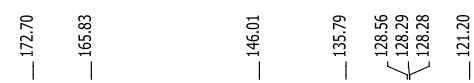

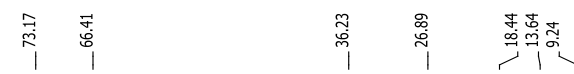
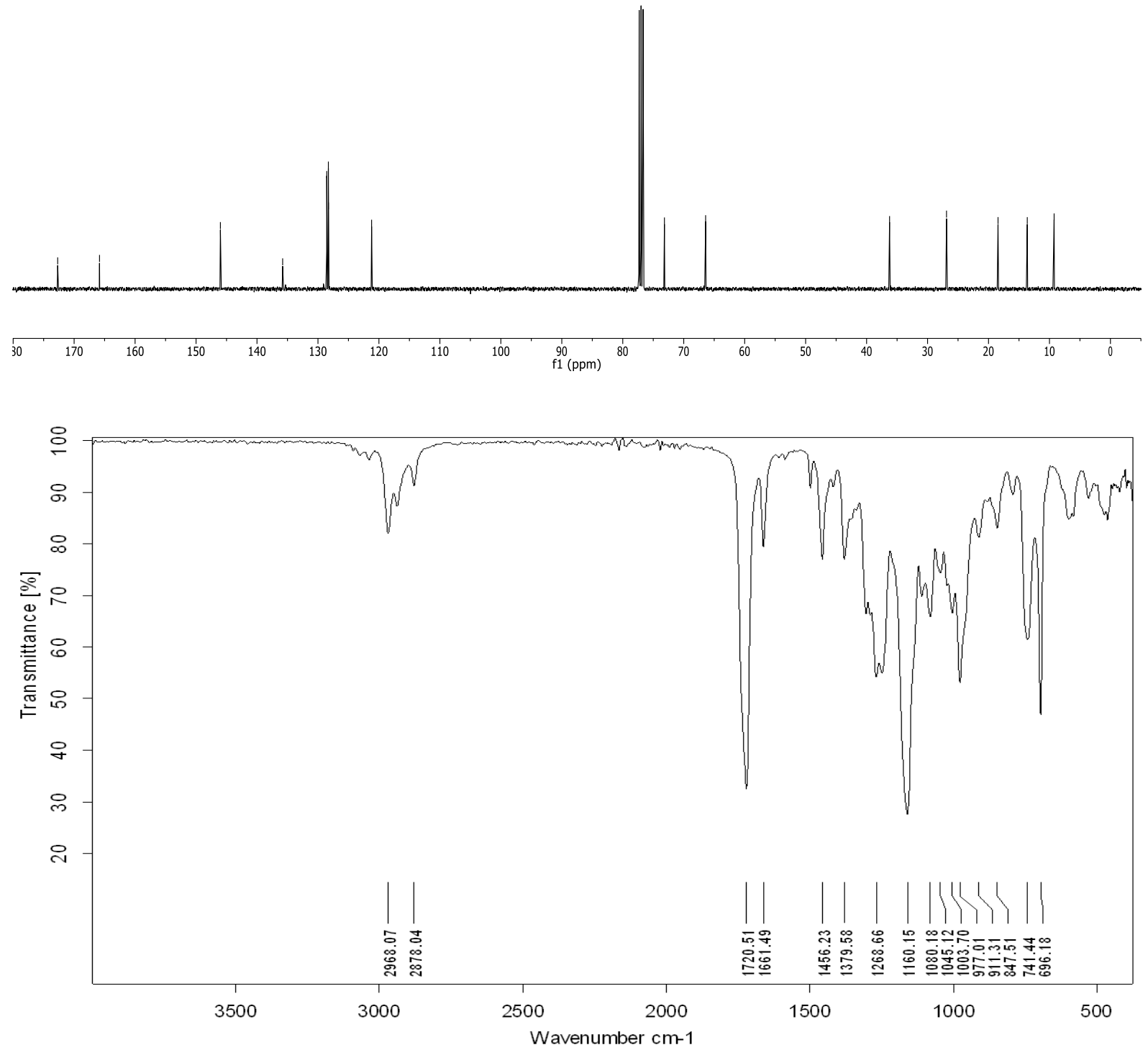
${ }^{1} \mathrm{H}-\mathrm{NMR}(300 \mathrm{MHz}),{ }^{13} \mathrm{C}-\mathrm{NMR}(75 \mathrm{MHz}): \mathrm{CDCl}_{3}$
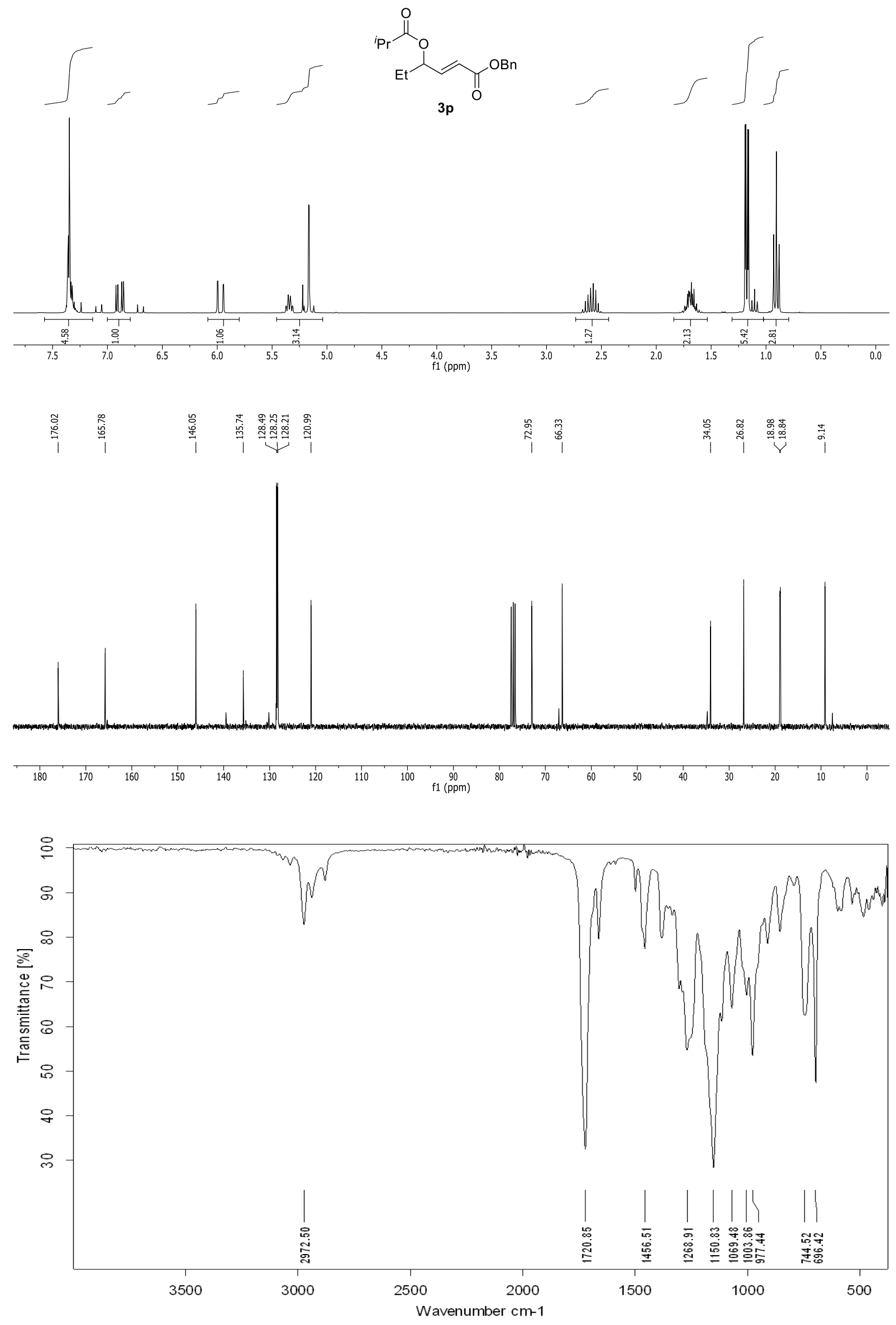
${ }^{1} \mathrm{H}-\mathrm{NMR}$ (300 MHz), ${ }^{13} \mathrm{C}-\mathrm{NMR}$ (75 MHz): $\mathrm{CDCl}_{3}$
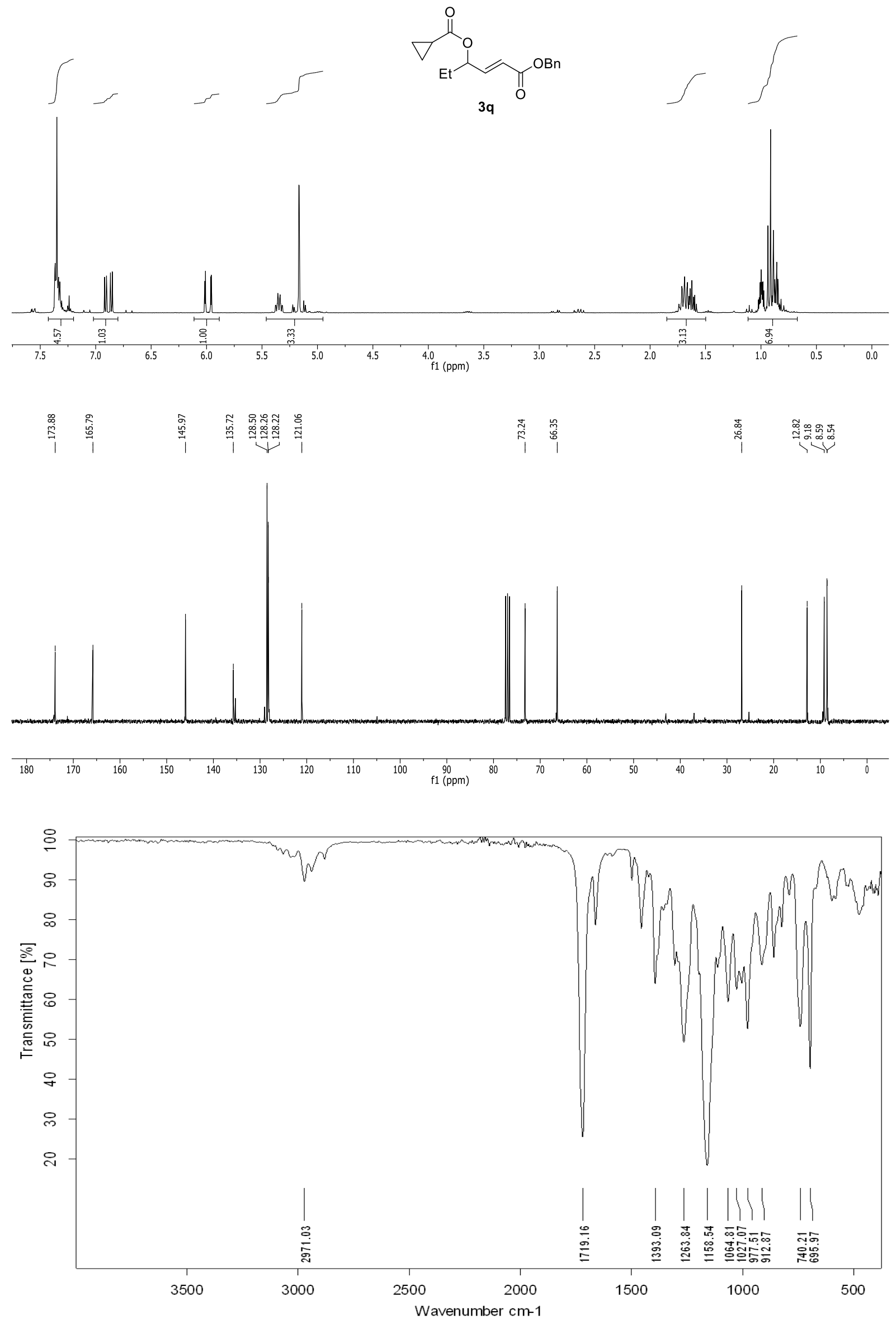
${ }^{1} \mathrm{H}-\mathrm{NMR}(400 \mathrm{MHz}),{ }^{13} \mathrm{C}-\mathrm{NMR}(101 \mathrm{MHz}): \mathrm{CDCl}_{3}$

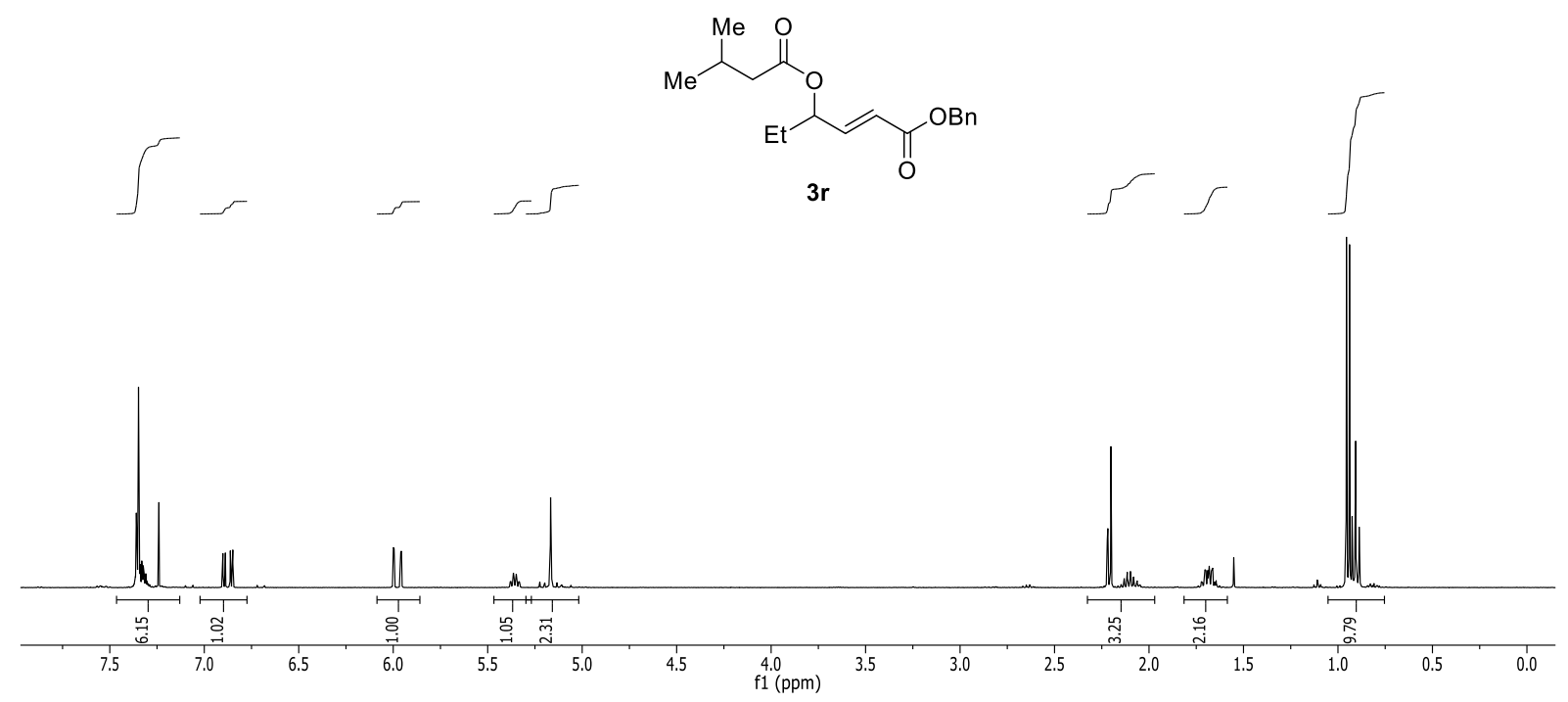

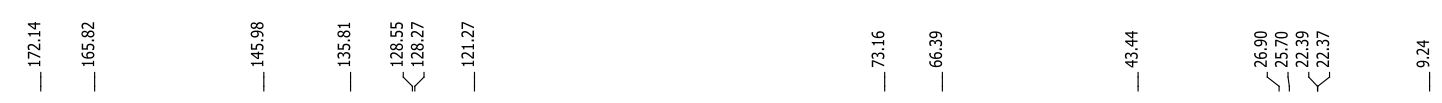
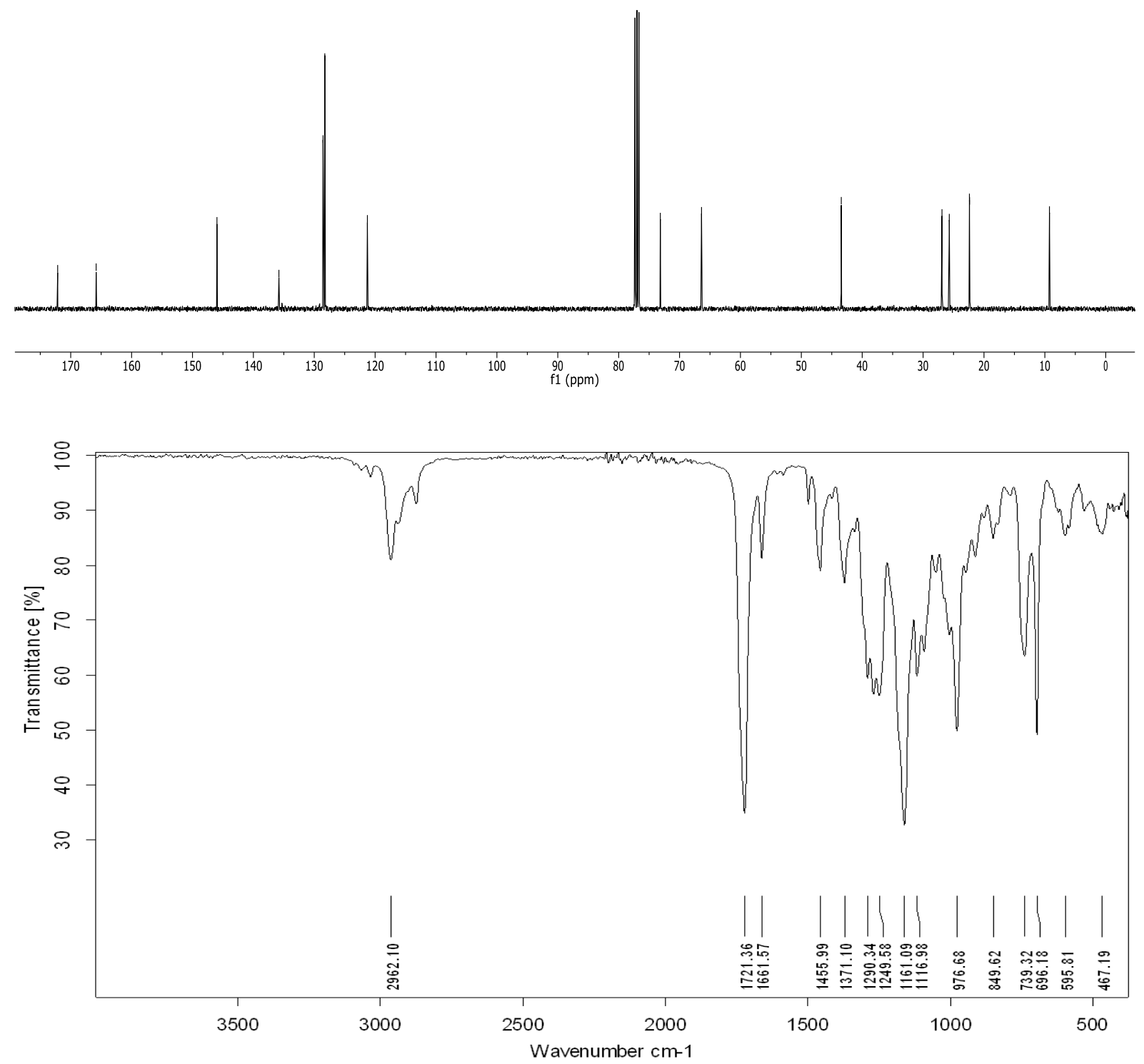
${ }^{1} \mathrm{H}-\mathrm{NMR}(400 \mathrm{MHz}),{ }^{13} \mathrm{C}$-NMR (101 MHz): $\mathrm{CDCl}_{3}$
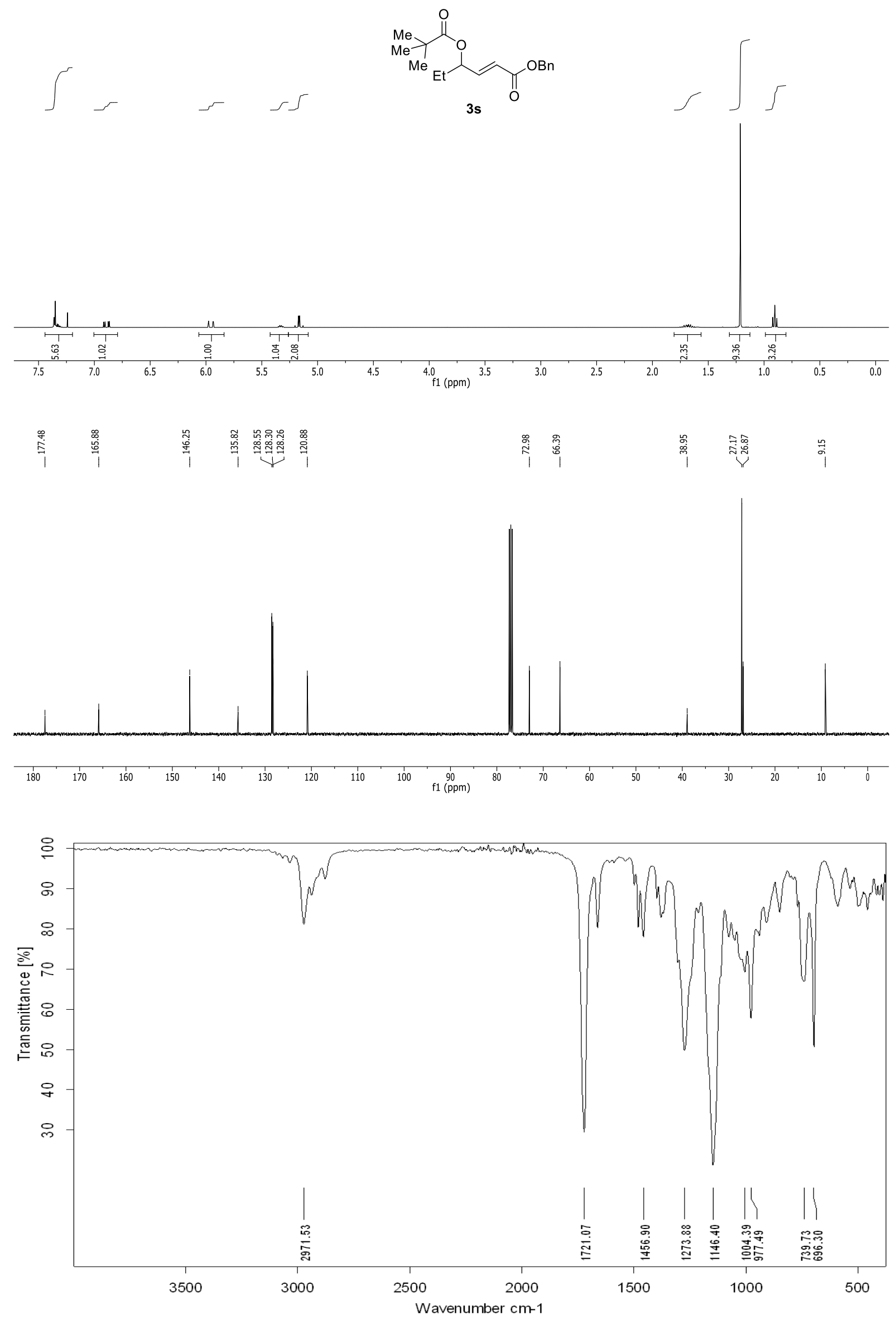
${ }^{1} \mathrm{H}-\mathrm{NMR}(300 \mathrm{MHz}),{ }^{13} \mathrm{C}$-NMR (75 MHz): $\mathrm{CDCl}_{3}$
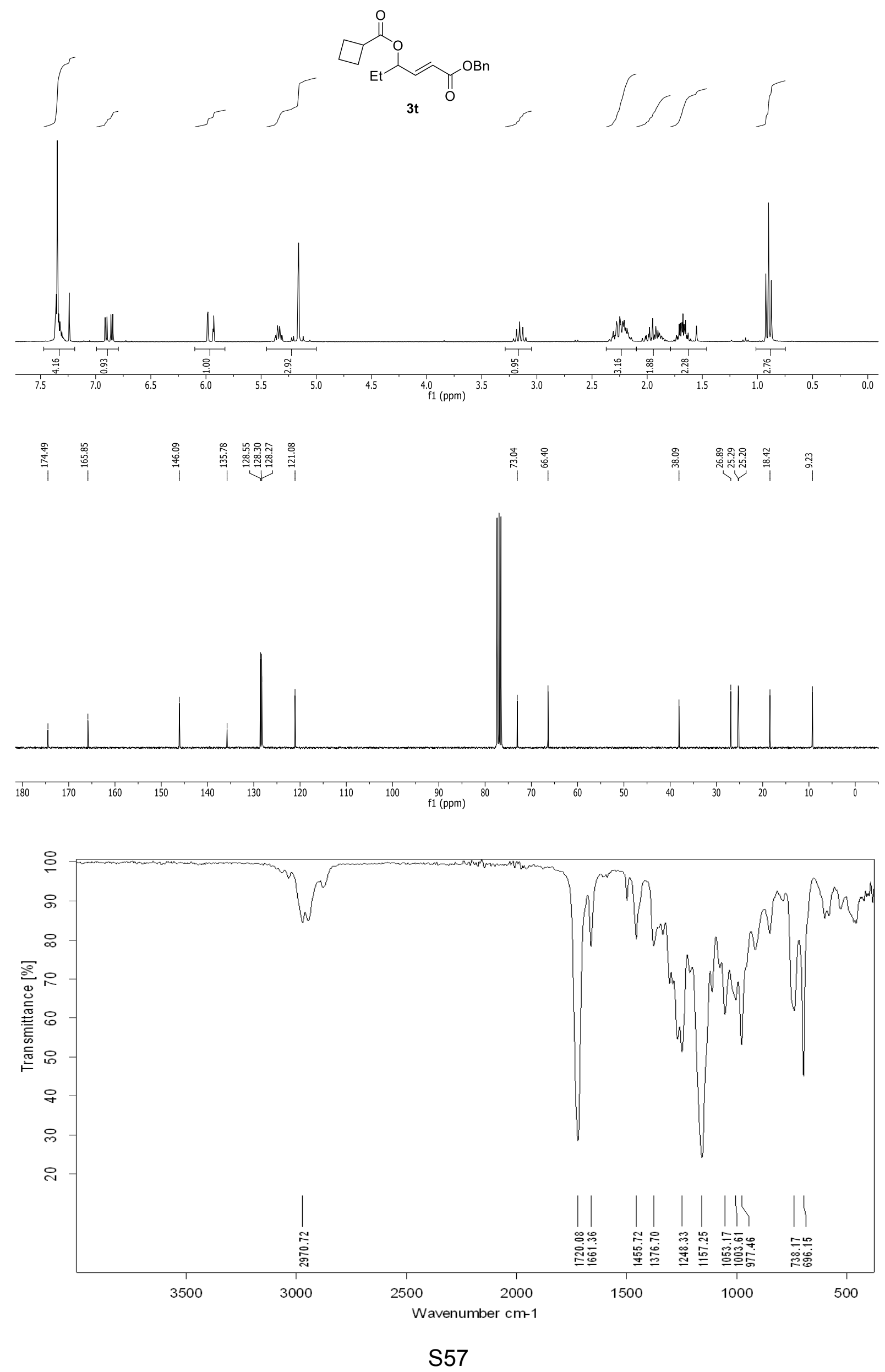
${ }^{1} \mathrm{H}-\mathrm{NMR}(400 \mathrm{MHz}),{ }^{13} \mathrm{C}$-NMR (101 MHz): $\mathrm{CDCl}_{3}$
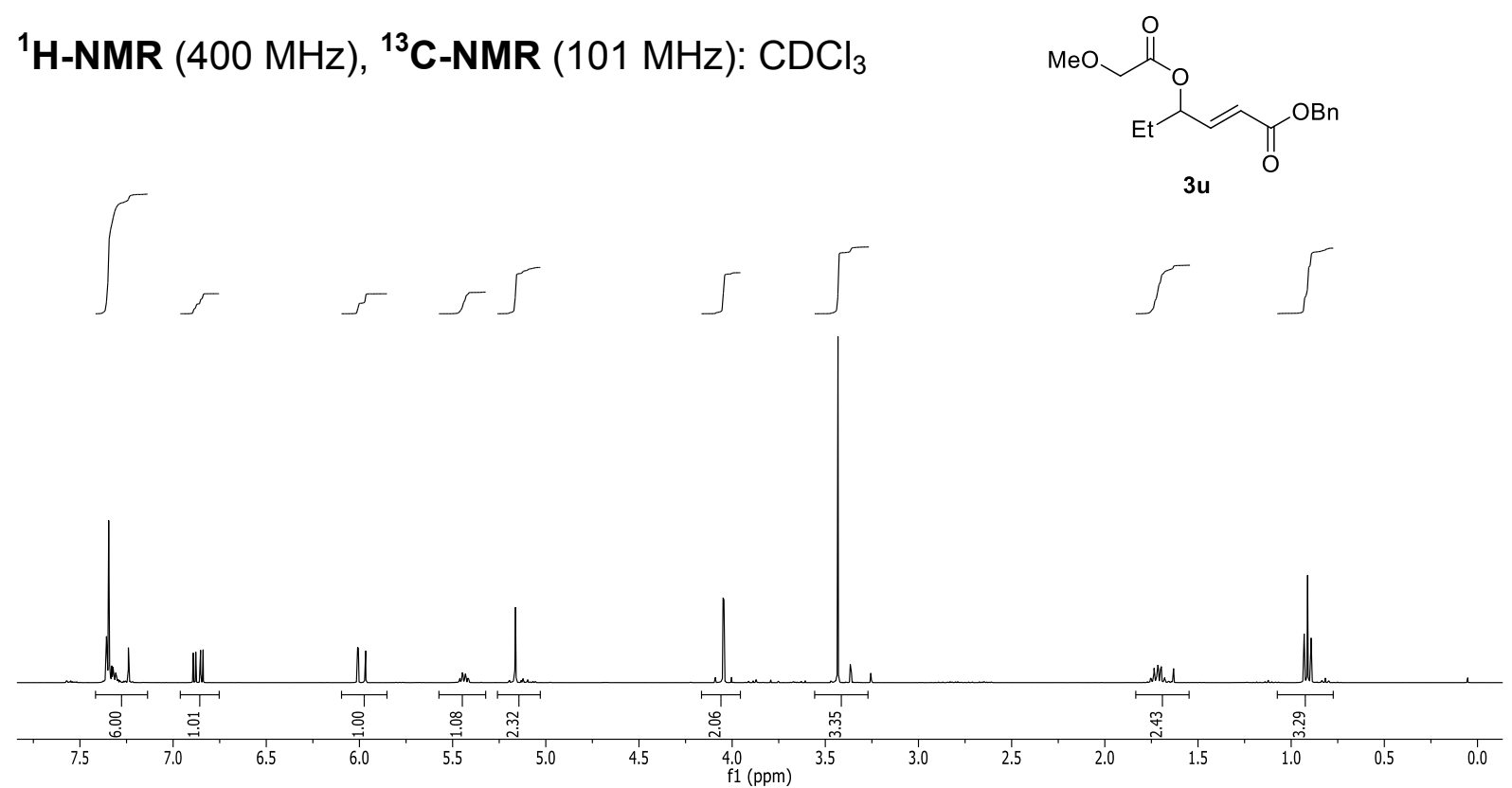

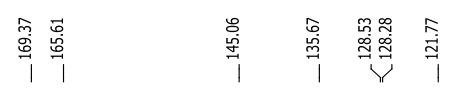

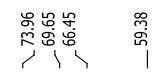

$\stackrel{\substack{0 \\ 1}}{\stackrel{\infty}{\sigma}}$
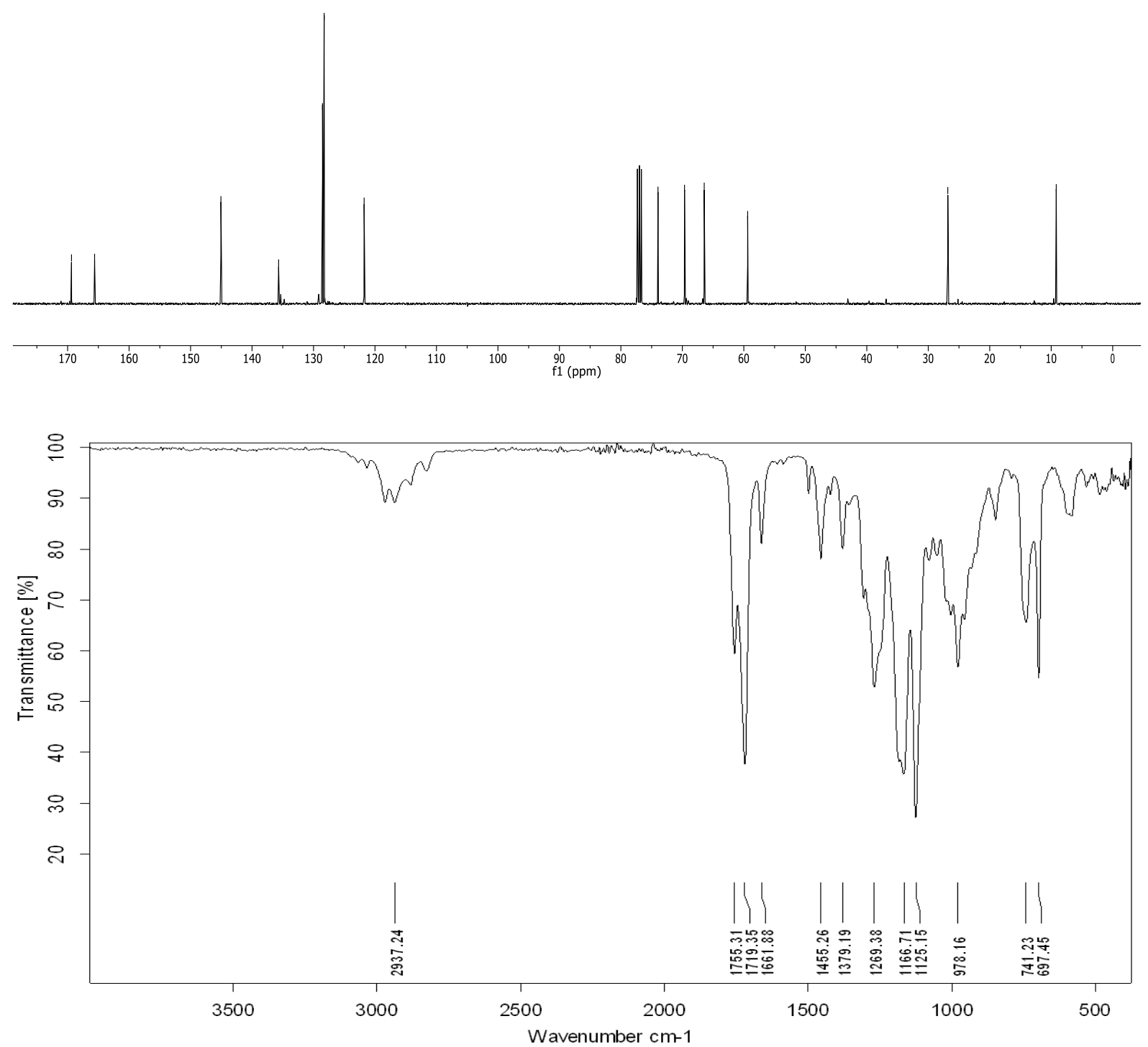
${ }^{1} \mathrm{H}-\mathrm{NMR}(300 \mathrm{MHz}),{ }^{13} \mathrm{C}-\mathrm{NMR}(75 \mathrm{MHz}): \mathrm{CDCl}_{3}$

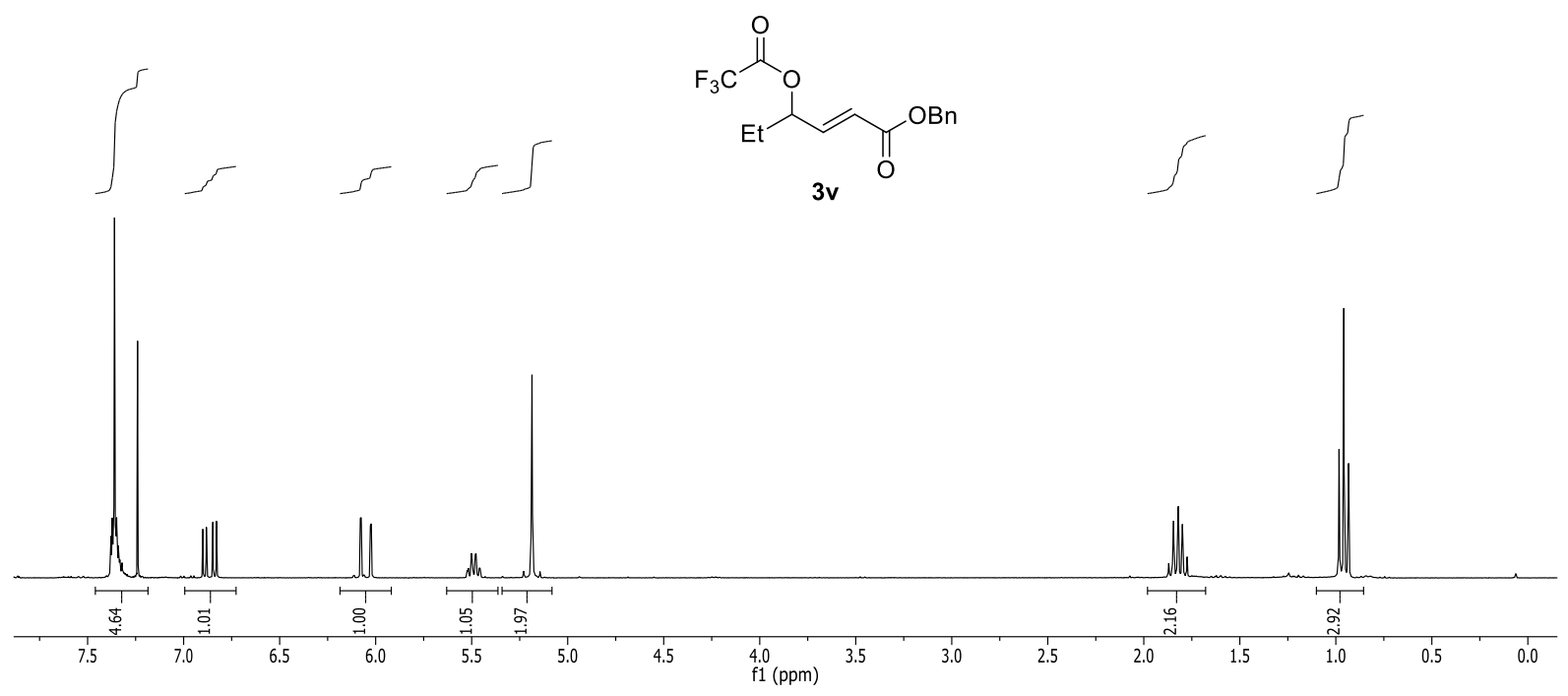

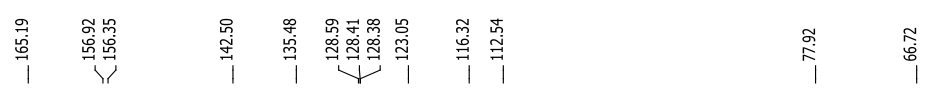

荢
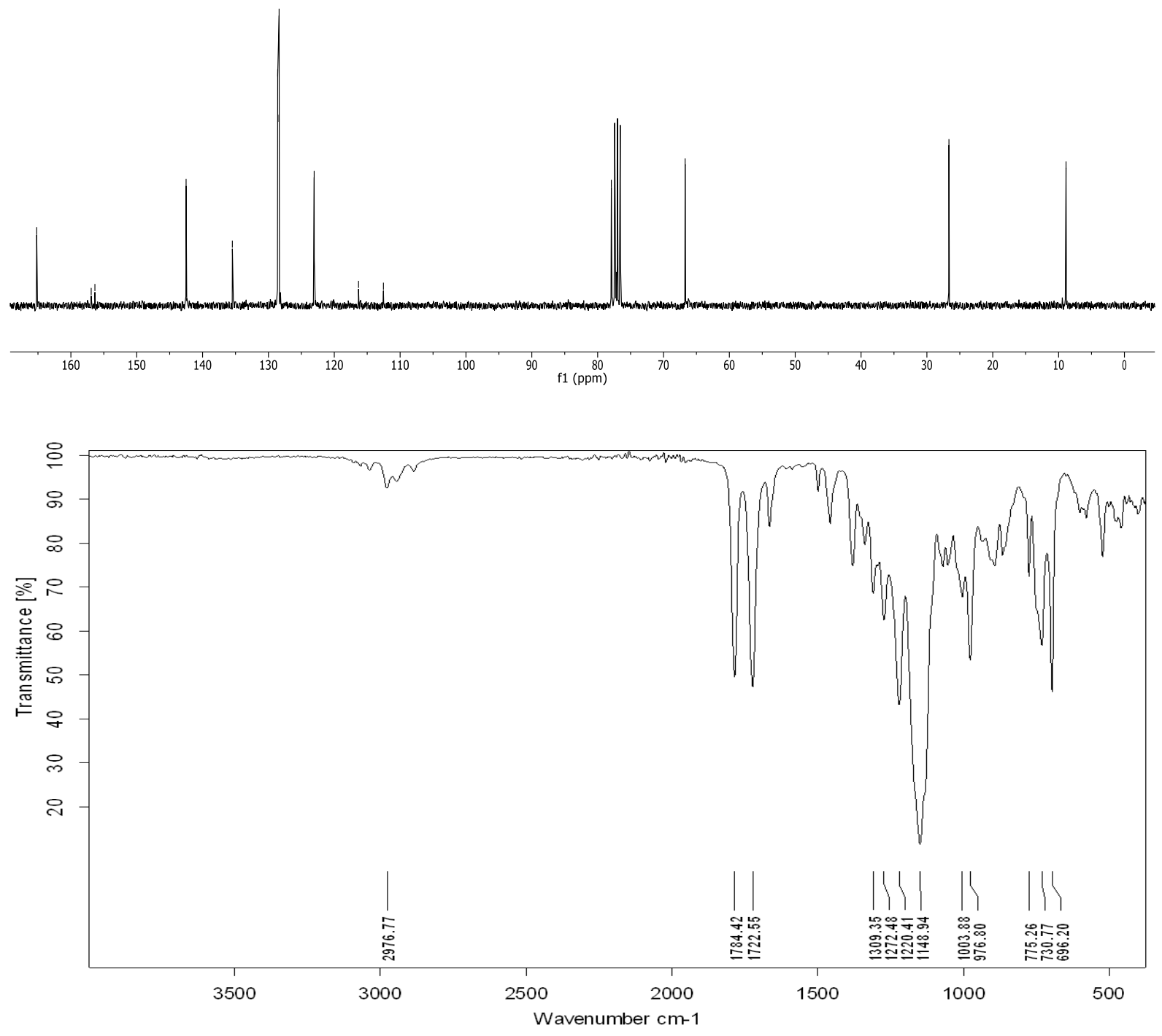

S59 
${ }^{1} \mathrm{H}$-NMR (300 MHz), ${ }^{13} \mathrm{C}$-NMR (75 MHz): $\mathrm{CDCl}_{3}$<smiles>CCC(C=CC(=O)OCc1ccccc1)OC(=O)CBr</smiles>

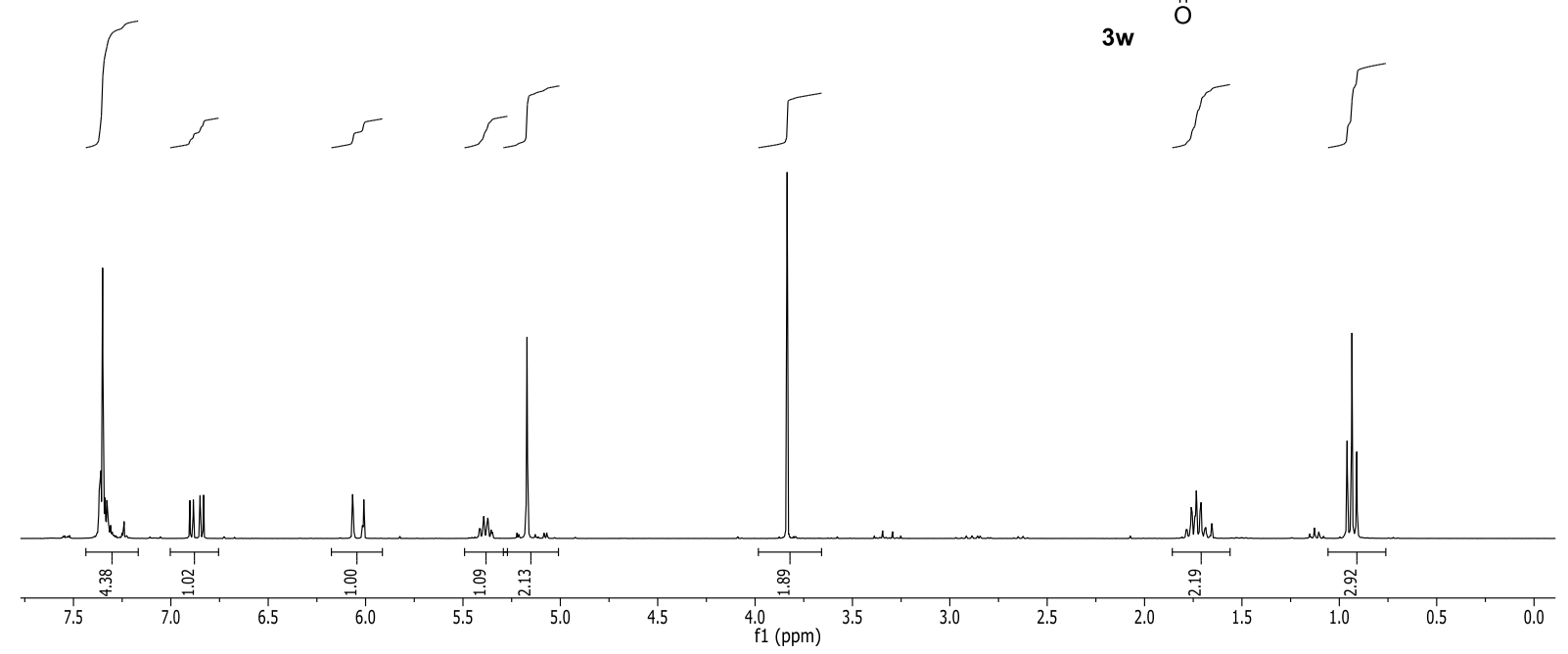

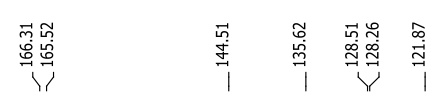

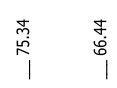

$\sqrt[3]{11}$
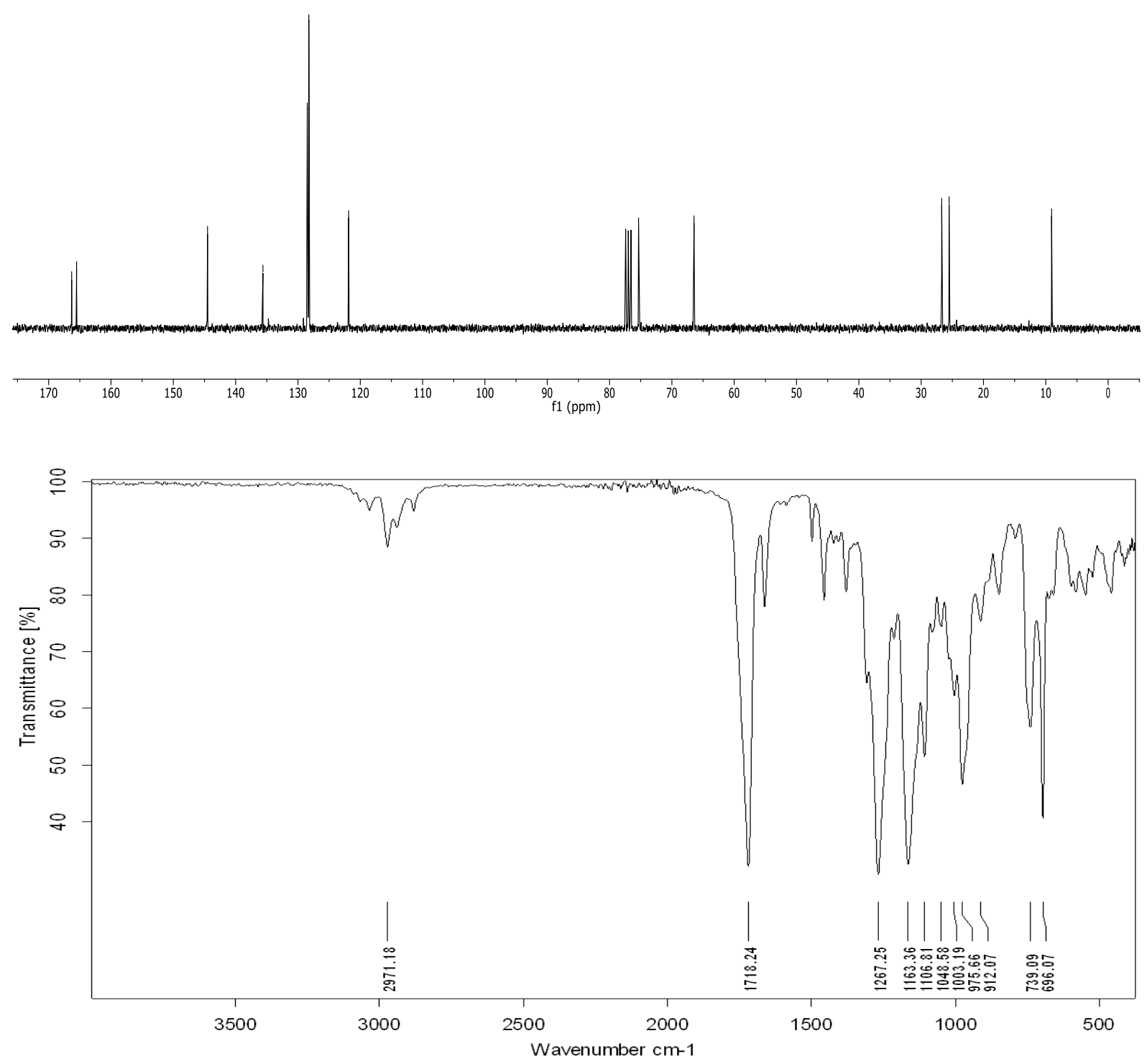

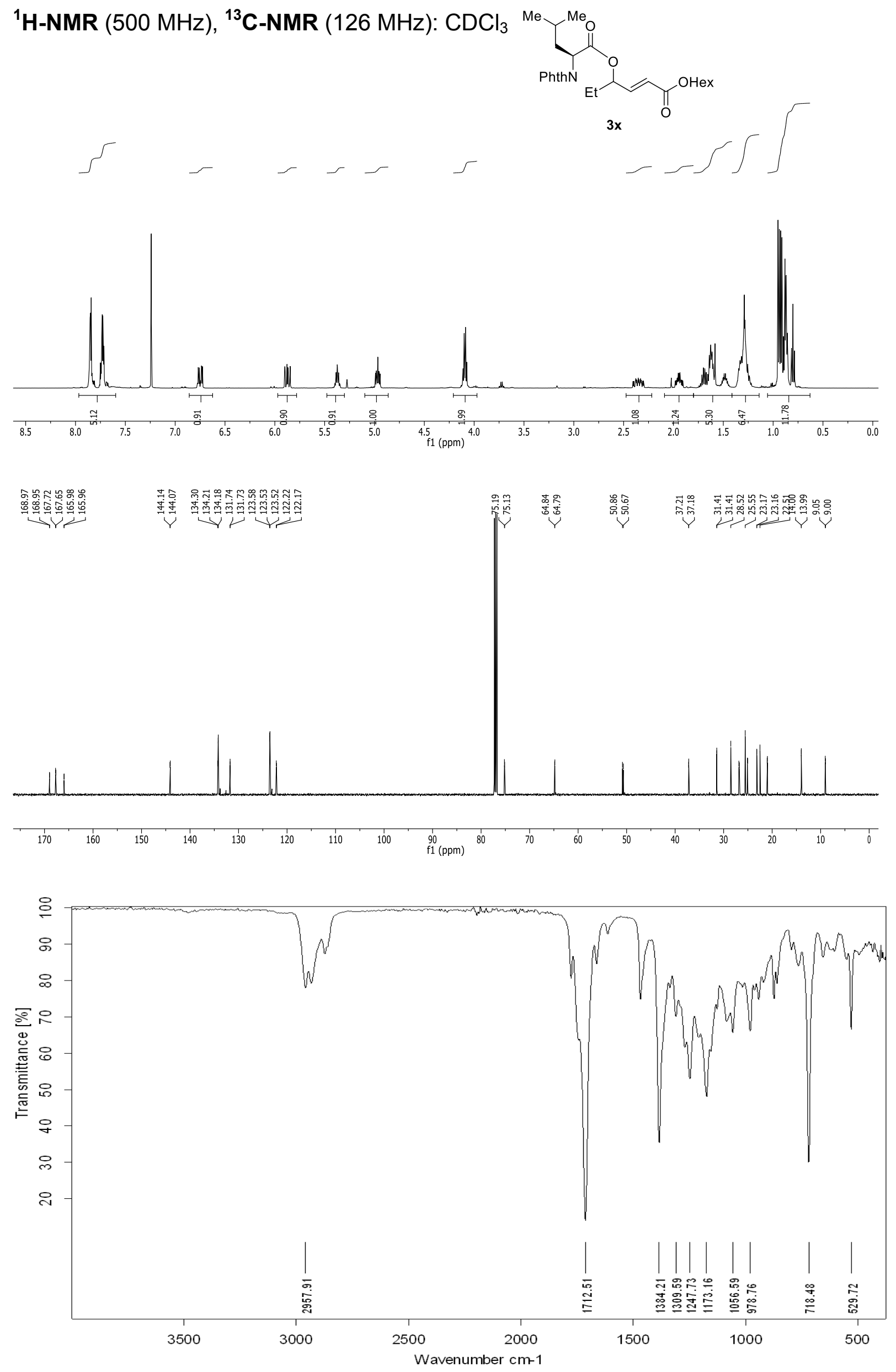
${ }^{1} \mathrm{H}$-NMR (400 MHz), ${ }^{13} \mathrm{C}$-NMR (101 MHz): $\mathrm{CDCl}_{3}$
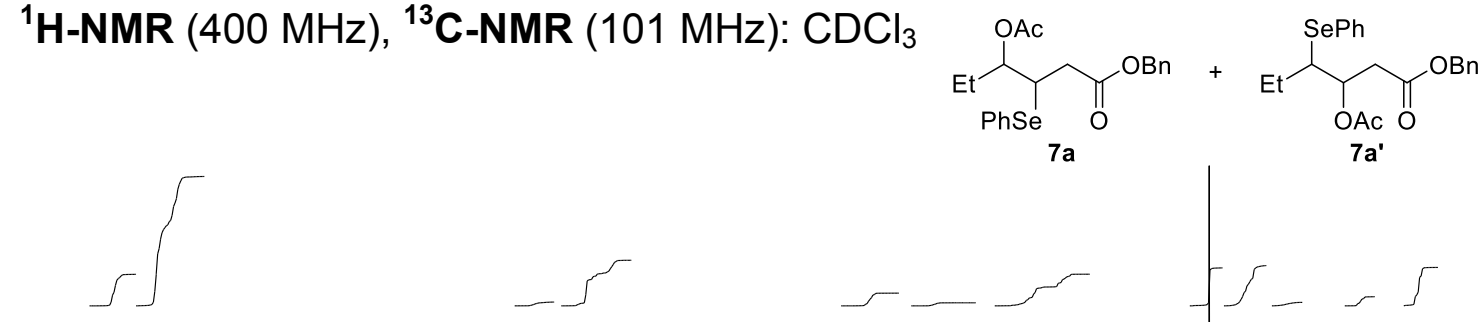

$7 a$

$7 a^{\prime}$

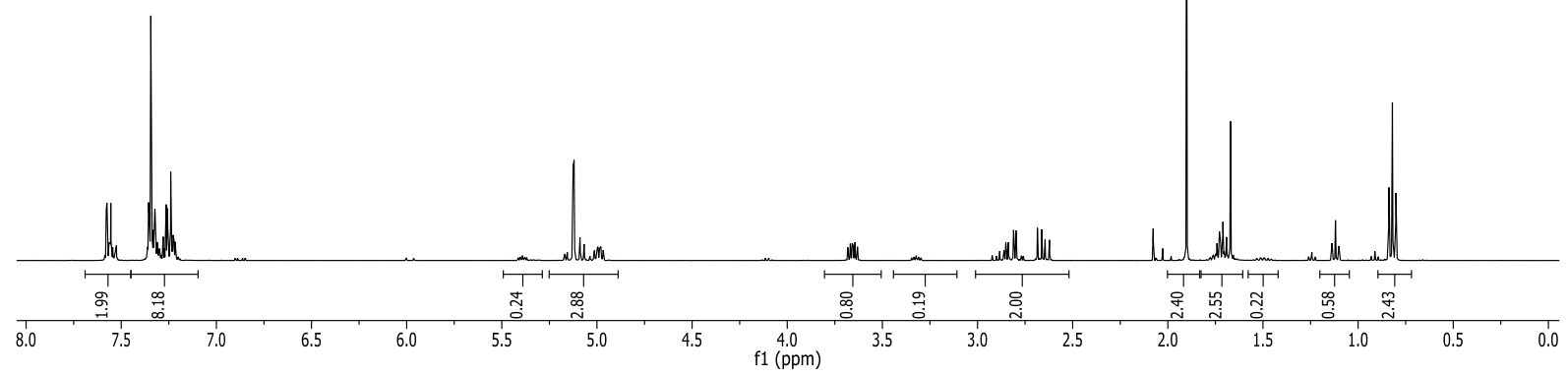

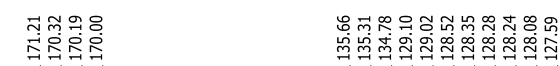

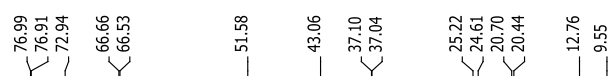
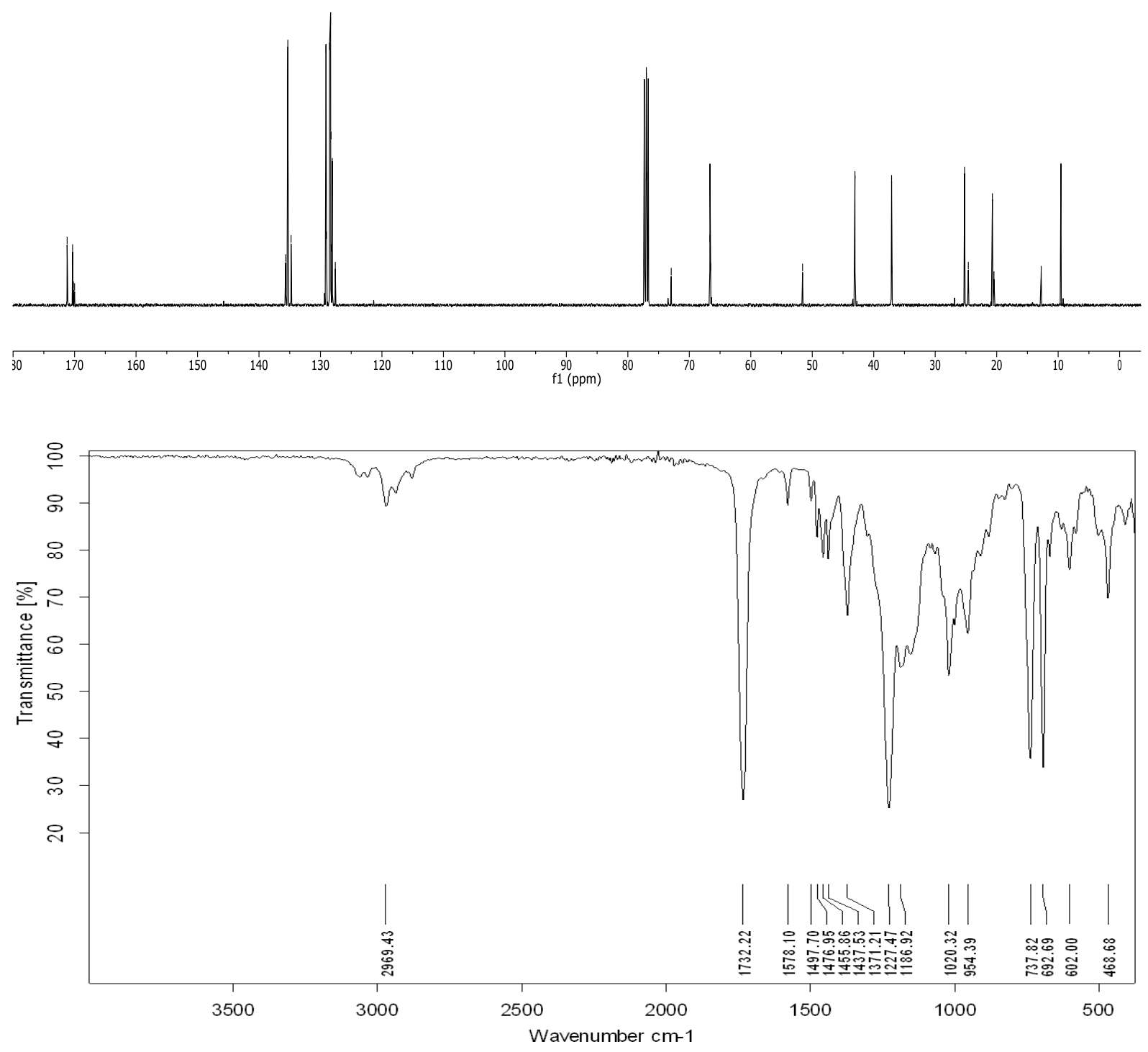